\title{
Phenazine-1,6-dicarboxamides: redox-responsive molecular switches
}

Jingwei Yin, Ali N. Khalilov, Pandi Muthupandi, Ruby Ladd, Vladimir B. Birman*

Department of Chemistry, Washington University, Campus Box 1134, One Brookings Drive,

Saint Louis, Missouri 63130

*birman@wustl.edu

SUPPORTING INFORMATION

\section{TABLE OF CONTENTS}

pages

1. General S-2

2. Synthesis of 1,1'-(phenazine-1,6-dicarbonyl)bis(3-methyl-1H-imidazol-3-ium) S-2 ditriflate (4)

3. Synthesis and reduction of model compound $6 \quad$ S-4

4. Synthesis of 2,7,9,9-tetramethyl-9H-xanthene-4,5-diamine (9) S-6

5. Synthesis and reduction of model compound $\mathbf{1 2} \quad$ S-7

6. Cyclic voltammetry study $\quad$ S-8

$\begin{array}{lr}\text { 7. Computer modeling } & \text { S-10 }\end{array}$

8. Gibbs free energy of the double flip $\quad$ S-11

9. 1D NMR spectra $\quad \mathbf{S - 1 2}$

10. 2D NMR spectra $\quad$ S-28

11. X-Ray Crystallographic data for $\mathbf{1 2} \quad$ S-32

12 References $\quad$ S-40 


\section{GENERAL}

All reagents were obtained commercially and used as received unless specified otherwise. All reactions were carried out under argon, unless noted otherwise. Hydrogenations were carried out by first charging the reaction vessel with solid starting materials and palladium catalyst, then purging with argon, then adding the reaction solvents, and finally purging the vessel with hydrogen. Note: mixtures of organic vapors or hydrogen with air have been observed to ignite spontaneously upon contact with an active palladium catalyst. Solvents used for chromatography were ACS or HPLC grade. Reactions were monitored by thin layer chromatography (TLC) and by $1 \mathrm{H}$ NMR. Uniplate HLF $(250 \mu \mathrm{m})$ silica gel plates were used for TLC analyses. Flash column chromatography was performed over Sorbent Technologies silica gel (40-63 mm). ${ }^{1} \mathrm{H},{ }^{13} \mathrm{C}$ and ${ }^{19} \mathrm{~F}$ NMR spectra were recorded on a Mercury $300 \mathrm{MHz}$ spectrometer and DD2 $500 \mathrm{MHz}$ and $600 \mathrm{MHz}$ Agilent spectrometers. The chemical shifts are reported as $\delta$ values (ppm) relative to TMS using the residual $\mathrm{CHCl}_{3}$ peak $(7.26 \mathrm{ppm})$ as the reference. Melting points were measured on a Stuart SMP10 melting point apparatus. High-Resolution mass spectral analyses were performed at Washington University MS Center on a Bruker MaXis QTOF mass spectrometer using Electrospray Ionization (ESI) and Atmospheric Pressure Chemical Ionization (APCI) methods. Infrared spectrums were recorded on a Bruker Alpha Platinum-ATR spectrophotometer.

\section{SYNTHESIS OF 1,1'-(PHENAZINE-1,6-DICARBONYL)BIS(3-METHYL-1H-IMIDAZOL-3-IUM) DITRIFLATE (4).}

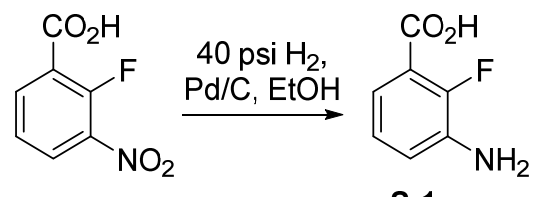

S-1

2a. 3-amino-2-fluorobenzoic acid (S-1). ${ }^{1}$ A mixture of 2-fluoro-3-nitrobenzoic acid (5.0 g, $\left.27 \mathrm{mmol}\right), 10$ $\mathrm{wt} \% \mathrm{Pd} / \mathrm{C}(50 \mathrm{mg}, 0.05 \mathrm{mmol})$ and $50 \mathrm{~mL}$ of EtOH was hydrogenated under $40 \mathrm{psi}$ of pressure in a Parr shaker for $4 \mathrm{~h}$. The mixture was filtered through Celite, dried with $\mathrm{Na}_{2} \mathrm{SO}_{4}$, and rotary evaporated to afford the product as a brown-pink solid, which was essentially pure by ${ }^{1} \mathrm{H}$ NMR. (4.1 g, 98\% yield). ${ }^{1} \mathbf{H}$ NMR $\left(600 \mathrm{MHz}, \mathrm{CD}_{3} \mathrm{OD}\right) \delta 4.95(\mathrm{br}, \mathrm{s}, 3 \mathrm{H}), 6.93-6.96(\mathrm{t}, \mathrm{J}=9 \mathrm{~Hz}, 1 \mathrm{H}), 7.00-7.02(\mathrm{t}, \mathrm{J}=6 \mathrm{~Hz}, 1 \mathrm{H}), 7.13(\mathrm{t}, \mathrm{J}=9$ $\mathrm{Hz}, 1 \mathrm{H}) .{ }^{13} \mathrm{C}$ NMR $\left(151 \mathrm{MHz}, \mathrm{CD}_{3} \mathrm{OD}\right) \delta 120.76,122.08-122.12(\mathrm{~d}, \mathrm{~J}=6 \mathrm{~Hz}), 124.99-125.02$ (d, J=4.5 Hz), 138.34-138.43 (d, J=13.5 Hz), 151.40, 153.07, 168.38. ${ }^{19} \mathbf{F}$ NMR $\left(282 \mathrm{MHz}, \mathrm{CD}_{3} \mathrm{OD}\right) \delta-125.76--125.78$ (m). IR $\left(\mathrm{cm}^{-1}\right): 1683,1485,1417,1296,745$; HRMS (ESI-TOF) $\mathrm{m} / \mathrm{z}$ : calcd for $\left[\mathrm{C}_{7} \mathrm{H}_{6} \mathrm{FNO}_{2}+\mathrm{H}\right]^{+}, 156.0460$; found, 156.0449 . $\mathrm{mp:} 167-169^{\circ} \mathrm{C}$.

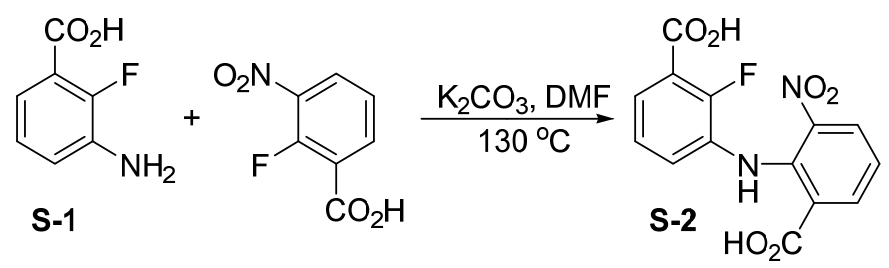

2b. 2-((3-carboxy-2-fluorophenyl)amino)-3-nitrobenzoic acid (S-2). A mixture of S-1 (4.1 g, $26 \mathrm{mmol})$, 2-fluoro-3-nitrobenzoic acid (5.0 g, $27 \mathrm{mmol}), \mathrm{K}_{2} \mathrm{CO}_{3}(9.3 \mathrm{~g}, 56 \mathrm{mmol})$ and DMF (100 mL) was stirred at $130{ }^{\circ} \mathrm{C}$ for $12 \mathrm{~h}$. After cooling, the reaction mixture was quenched with $50 \mathrm{~mL}$ of $6 \mathrm{M}$ aqueous $\mathrm{HCl}$ and extracted with EtOAc $(3 \times 100 \mathrm{~mL})$. The combined organic phase was dried over $\mathrm{MgSO}_{4}$ and rotary evaporated. The residue was purified by flash chromatography (hexane/EtOAc/AcOH 66:33:1) to afford the product as a yellow solid (6.4 g, 77\% yield). ${ }^{1} \mathbf{H}$ NMR $\left(500 \mathrm{MHz}, \mathrm{CD}_{3} \mathrm{OD}\right) \delta 4.92(\mathrm{br}, \mathrm{s}, 3 \mathrm{H}), 7.06-7.11$ $(\mathrm{m}, 3 \mathrm{H}), 7.55(\mathrm{~m}, 3 \mathrm{H}), 8.07-8.09(\mathrm{~d}, \mathrm{~J}=10 \mathrm{~Hz} 1 \mathrm{H}), 8.30-8.32(\mathrm{~d}, \mathrm{~J}=10 \mathrm{~Hz} 1 \mathrm{H}) .{ }^{13} \mathbf{C}$ NMR $(126 \mathrm{MHz}$, $\left.\mathrm{CD}_{3} \mathrm{OD}\right) \delta 120.38,121.48,124.10,124.86-124.90$ (d, J=5 Hz), 127.45, 132.14-132.18 (d, J=5 Hz), 132.28, 
138.22, 140.83, 141.47, 154.50, 156.55, 167.27, 170.25. ${ }^{19} \mathbf{F}$ NMR $\left(282 \mathrm{MHz}, \mathrm{CDCl}_{3}\right) \delta 27.32$ - $27.34(\mathrm{~m})$. IR $\left(\mathrm{cm}^{-1}\right): 2908,1682,1260,934,774,753,739,720$; HRMS (ESI-TOF) $\mathrm{m} / \mathrm{z}$ : calcd for $\left[\mathrm{C}_{14} \mathrm{H}_{9} \mathrm{FN}_{2} \mathrm{O}_{6}+\right.$ $\mathrm{Na}]^{+} 343.0342$, found 343.0327 . mp: $230-231^{\circ} \mathrm{C}$.

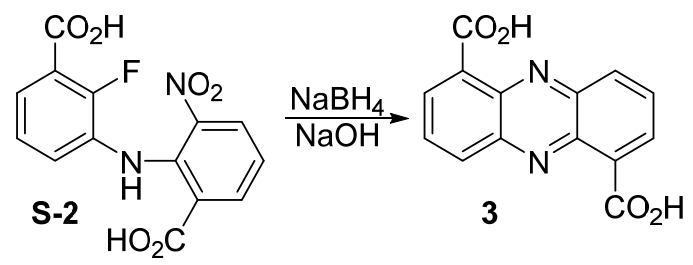

2c. Phenazine-1,6-dicarboxylic acid (3). ${ }^{2}$ A solution of S-2 $(6.4 \mathrm{~g}, 20 \mathrm{mmol})$ in $400 \mathrm{~mL}$ of $3 \mathrm{M}$ aqueous $\mathrm{NaOH}$ was treated with solid $\mathrm{NaBH}_{4}(20.0 \mathrm{~g}, 535 \mathrm{mmol})$ added in small portions at room temperature, then refluxed overnight $(10 \mathrm{~h})$ and cooled to room temperature. The resulting yellow suspension was brought to pH 7 with $6 \mathrm{M}$ aqueous $\mathrm{HCl}$, at which point the precipitate turned green. The suspension was treated with $3 \%$ aqueous $\mathrm{H}_{2} \mathrm{O}_{2}(100 \mathrm{~mL})$ and sparged with air overnight. The precipitate, which at this point was brown, was filtered off, rinsed on the filter with deionized water, and dried in a desiccator. The dry product was light-brown. (4.5 g, 84\% yield). ${ }^{1} \mathbf{H}$ NMR $\left(300 \mathrm{MHz}\right.$, as disodium salt, $\left.\mathrm{KOH}, \mathrm{D}_{2} \mathrm{O}\right) \delta 7.91(\mathrm{~s}, 4 \mathrm{H}), 8.24(\mathrm{~s}$, $2 \mathrm{H}) .{ }^{13} \mathbf{C}$ NMR $\left(126 \mathrm{MHz}, \mathrm{D}_{2} \mathrm{O}\right) \delta 126.72,129.51,129.56,137.92,138.23,141.04,174.50$. IR $\left(\mathrm{cm}^{-1}\right): 1740$, 1394, 1230, 858, 754; HRMS (ESI-TOF) m/z: calcd for: $\left[\mathrm{C}_{14} \mathrm{H}_{8} \mathrm{~N}_{2} \mathrm{O}_{4}+\mathrm{Na}\right]^{+}$291.0376; found 291.1466. mp: $>300^{\circ} \mathrm{C}$.<smiles>O=C(O)c1cccc2nc3c(C(=O)O)cccc3nc12</smiles><smiles>O=C(c1cccc2nc3c(C(=O)n4ccnc4)cccc3nc12)n1ccnc1</smiles>

3d. 1,1'-(phenazine-1,6-dicarbonyl)bis-imidazole (S-3). ${ }^{3}$ A $10 \mathrm{~mL}$ centrifugation tube was charged with a stir bar, phenazine-1,6-dicarboxylic acid $3(1.2 \mathrm{~g}, 4.47 \mathrm{mmol})$ and $5 \mathrm{~mL}$ of DMF. The resulting green suspension was stirred at room temperature and treated with solid CDI (2.5 g, $15.4 \mathrm{mmol})$ added in small portions. The reaction mixture quickly turned deep-red. After stirring overnight (ca. $12 \mathrm{~h}$ ), the red color faded and a beige precipitate was observed. The mixture was diluted with $5 \mathrm{~mL}^{\text {of }} \mathrm{Et}_{2} \mathrm{O}$, stirred vigorously, and centrifuged. The supernatant was removed carefully via syringe. The procedure was repeated 4 more times leaving essentially pure product as a pale yellowish-beige powder, which was dried by rotary evaporation in the same tube $(1.56 \mathrm{~g}, 94 \%$ yield $) .{ }^{1} \mathbf{H}$ NMR $\left(600 \mathrm{MHz}, \mathrm{CDCl}_{3}\right) \delta 7.16(\mathrm{~s}, 2 \mathrm{H}), 7.52(\mathrm{~s}, 2 \mathrm{H})$, $7.86(\mathrm{~s}, 2 \mathrm{H}), 7.97-7.99(\mathrm{t}, \mathrm{J}=6 \mathrm{~Hz}, 2 \mathrm{H}), 8.14-8.16(\mathrm{~d}, \mathrm{~J}=12 \mathrm{~Hz}, 2 \mathrm{H}), 8.36-8.38(\mathrm{~d}, \mathrm{~J}=12 \mathrm{~Hz}, 2 \mathrm{H}){ }^{13} \mathbf{C}$ NMR $\left(151 \mathrm{MHz}, \mathrm{CDCl}_{3}\right) \delta 117.32,130.22,131.23,132.10,132.96,133.93,138.13,164.41$. IR $\left(\mathrm{cm}^{-1}\right): 1721$, 1537, 1410, 1383, 1288, 1237, 810, 748, 730, 677, 644; HRMS (ESI-TOF) m/z: calcd for $\left[\mathrm{C}_{20} \mathrm{H}_{12} \mathrm{~N}_{6} \mathrm{O}_{2}+\right.$ $\mathrm{H}]^{+}: 369.1095$, found 369.1085 . mp: $230{ }^{\circ} \mathrm{C}(\mathrm{dec})$.<smiles>O=C(c1cccc2nc3c(C(=O)n4ccnc4)cccc3nc12)n1ccnc1</smiles><smiles></smiles> 
3e. 1,1'-(phenazine-1,6-dicarbonyl)bis(3-methyl-1H-imidazol-3-ium) triflate (4). A suspension of S-3 $(1.56 \mathrm{~g}, 4.23 \mathrm{mmol})$ in $2 \mathrm{~mL}$ of $\mathrm{CH}_{2} \mathrm{Cl}_{2}$ stirring at room temperature in a centrifugation tube was treated with MeOTf $(1.3 \mathrm{~mL}, 11.84 \mathrm{mmol})$ added slowly dropwise. After stirring for $2 \mathrm{~h}$, the mixture was centrifuged and the supernatant removed via syringe. The precipitate was re-suspended in $5 \mathrm{~mL}$ of $\mathrm{CH}_{2} \mathrm{Cl}_{2}$ and the procedure repeated 3 more times. After drying by rotary evaporation in the same tube, the lightyellow microcrystalline product weighed $2.89 \mathrm{~g}$ (98\% yield) and was sufficiently pure for the next step. ${ }^{1} \mathbf{H}$ NMR $\left(300 \mathrm{MHz}, \mathrm{CD}_{3} \mathrm{CN}\right) \delta 3.89(\mathrm{~s}, 6 \mathrm{H}), 7.58-7.59(\mathrm{~m}, 2 \mathrm{H}), 8.03-8.04(\mathrm{~m}, 2 \mathrm{H}), 8.15-8.20\left(\mathrm{dd}, \mathrm{J}_{1}=9 \mathrm{~Hz}\right.$, $\left.\mathrm{J}_{2}=6 \mathrm{~Hz}, 2 \mathrm{H}\right), 8.40-8.44\left(\mathrm{dd}, \mathrm{J}_{1}=3 \mathrm{~Hz}, \mathrm{~J}_{2}=9 \mathrm{~Hz}, 2 \mathrm{H}\right), 8.48-8.50\left(\mathrm{dd}, \mathrm{J}_{1}=3 \mathrm{~Hz}, \mathrm{~J}_{2}=6 \mathrm{~Hz}, 2 \mathrm{H}\right), 9.08(\mathrm{br}$, $2 \mathrm{H}) .{ }^{13} \mathrm{C}$ NMR $\left(151 \mathrm{MHz}, \mathrm{CD}_{3} \mathrm{CN}\right) \delta 120.77,125.85,130.05,132.16,136.16,136.76,140.17,141.98$, 143.42, 163.94. IR $\left(\mathrm{cm}^{-1}\right): 1745,1256,1167,1028,878,749,637,618,517$; mp: $185^{\circ} \mathrm{C}(\mathrm{dec})$.

\section{SYNTHESIS AND REDUCTION OF MODEL COMPOUND 6.}

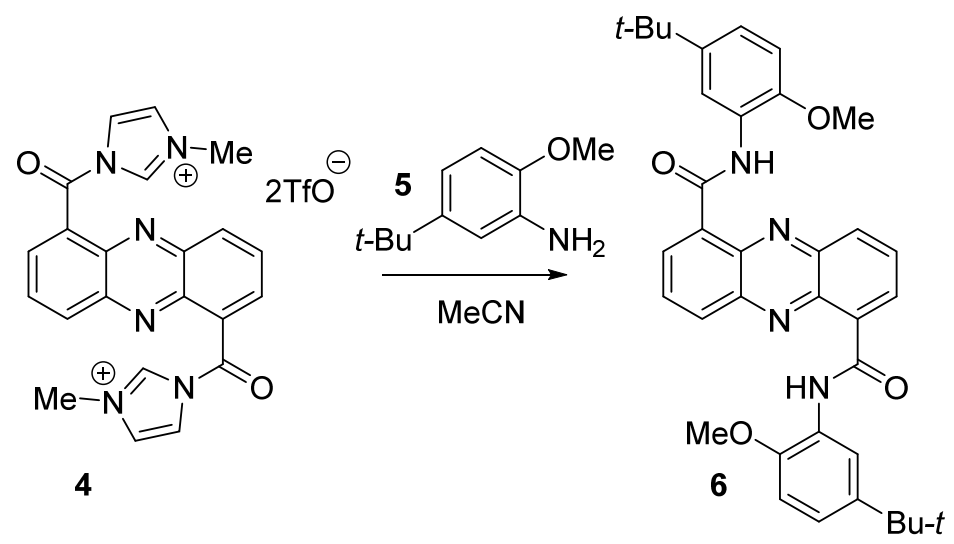

3a. N1,N6-bis(5-(tert-butyl)-2-methoxyphenyl)phenazine-1,6-dicarboxamide (6). To a suspension of 4 $(270 \mathrm{mg}, 0.39 \mathrm{mmol})$ in $1 \mathrm{~mL} \mathrm{MeCN}$ stirring at room temperature was slowly added a solution of 5-( $t$ butyl)-2-methoxyaniline 5 (170 $\mathrm{mg}, 0.93 \mathrm{mmol})$ in $1 \mathrm{~mL} \mathrm{MeCN}$. TLC indicated complete conversion after $30 \mathrm{~min}$. The reaction mixture was rotary evaporated, the residue taken up in $\mathrm{CH}_{2} \mathrm{Cl}_{2}$, rinsed with water, and dried over $\mathrm{MgSO}_{4}$. Flash chromatography (hexanes/EtOAc 2:1) afforded the product as a red-orange solid (226 mg, 98\% yield). ${ }^{1} \mathbf{H}$ NMR $\left(600 \mathrm{MHz}, \mathrm{CD}_{2} \mathrm{Cl}_{2}\right) \delta 1.41(\mathrm{~s}, 18 \mathrm{H}), 4.13(\mathrm{~s}, 6 \mathrm{H}), 7.02-7.03$ (d, 2H, J = 6 $\mathrm{Hz}), 7.19-7.20(\mathrm{~d}, 2 \mathrm{H}, \mathrm{J}=6 \mathrm{~Hz}), 8.11-8.13(\mathrm{t}, 2 \mathrm{H}, \mathrm{J}=6 \mathrm{~Hz}), 8.52-8.53(\mathrm{~d}, 2 \mathrm{H}, \mathrm{J}=6 \mathrm{~Hz}), 8.91(\mathrm{~s}, 2 \mathrm{H})$, $9.11(\mathrm{~s}, 2 \mathrm{H}), 13.12(\mathrm{~s}, 2 \mathrm{H}) .{ }^{13} \mathrm{C}$ NMR $\left(151 \mathrm{MHz}, \mathrm{CD}_{2} \mathrm{Cl}_{2}\right) \delta 31.92,34.93,56.77,110.57,119.00,121.09$, 128.81, 130.70, 131.97, 133.37, 136.56, 140.77, 141.99, 144.56, 147.73, 162.47. IR $\left(\mathrm{cm}^{-1}\right): 2950,1662$, 1591, 1528, 1255, 1220, 1178, 1139, 1030, 746. HRMS (ESI-TOF) m/z: calcd for: $\left[\mathrm{C}_{36} \mathrm{H}_{38} \mathrm{~N}_{4} \mathrm{O}_{4}+\mathrm{H}\right]^{+}$: 591.2966, found $591.2940 . \mathbf{m p :}>300{ }^{\circ} \mathrm{C}$.<smiles></smiles> 
3b. N1,N6-bis(5-(tert-butyl)-2-methoxyphenyl)-5,10-dihydrophenazine-1,6-dicarboxamide (7). A pressure tube with a side arm was charged with 6 (34 mg, $0.058 \mathrm{mmol}), 10 \mathrm{wt} \% \mathrm{Pd} / \mathrm{C}(5 \mathrm{mg}, 0.005 \mathrm{mmol})$, THF $(0.7 \mathrm{~mL})$ and $\mathrm{MeOH}(0.7 \mathrm{~mL})$ (See Note in Section 1) and connected to a hydrogen reservoir. The mixture was stirred magnetically under $20 \mathrm{psi}$ of hydrogen pressure at room temperature for $2 \mathrm{~h}$. The solvents were removed by evacuating the apparatus through the side arm and then purging it with argon. Degassed $\mathrm{CDCl}_{3}(0.7 \mathrm{~mL})$ was added via syringe under an argon blanket. The resulting suspension was withdrawn via syringe and filtered through a PTFE syringe filter $(0.45 \mu \mathrm{m}$ porosity $)$ into an NMR tube filled with argon. A sample thus prepared contained ca. 95\% pure dihydrophenazine 7 and was stable for 2 days in a capped NMR tube. Upon deliberate exposure to air, it converted back into 6 within 2 h. ${ }^{1} \mathbf{H}$ NMR $\left(600 \mathrm{MHz}, \mathrm{CD}_{2} \mathrm{Cl}_{2}\right) \delta 1.35(\mathrm{~s}, 18 \mathrm{H}), 3.90(\mathrm{~s}, 6 \mathrm{H}), 6.21-6.22(\mathrm{~d}, 2 \mathrm{H}, \mathrm{J}=6 \mathrm{~Hz}), 6.38-6.41 \quad(\mathrm{t}, 2 \mathrm{H}, \mathrm{J}=6$ $\mathrm{Hz}), 6.68-6.69$ (d, 2H, J = 6 Hz), $6.87-6.89$ (d, 2H, J = 6 Hz), $7.10-7.12$ (dd, 2H, J $=6 \mathrm{~Hz}, \mathrm{~J}_{2}=6 \mathrm{~Hz}$ ), 8.39 (s, 2H), $8.42-8.43(\mathrm{~d}, 2 \mathrm{H}, \mathrm{J}=6 \mathrm{~Hz}), 9.13(\mathrm{~s}, 2 \mathrm{H}) .{ }^{13} \mathrm{C} \mathrm{NMR}\left(151 \mathrm{MHz}, \mathrm{CD}_{2} \mathrm{Cl}_{2}\right) \delta 26.15,30.65,31.85$, $34.88,56.45,68.32,110.09,113.65,114.70,117.98,119.01,120.48,120.97,127.54,134.63,137.71$, $144.34,146.97,166.91$.

\section{SYNTHESIS OF 2,7,9,9-TETRAMETHYL-9H-XANTHENE-4,5-DIAMINE (9)}

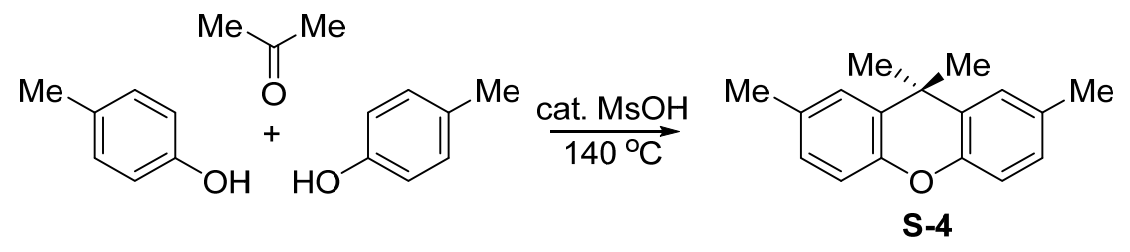

4a. 2,7,9,9-tetramethyl-9H-xanthene (S-4). Prepared via a modified patent procedure. ${ }^{4}$ A mixture of $p$ cresol $(20.0 \mathrm{~g}, 185 \mathrm{mmol})$ and methanesulfonic acid $(0.45 \mathrm{~mL}, 6.9 \mathrm{mmol})$ was stirred at $140{ }^{\circ} \mathrm{C}$ under $\mathrm{N}_{2}$ in a $100 \mathrm{~mL}, 3$-necked flask equipped with a vertical reflux condenser and a thermometer. Using a syringe pump, acetone ( $4 \mathrm{~mL}, 54 \mathrm{mmol}$ ) was added over the course of $8 \mathrm{~h}$ via a steel needle passing through the condenser and immersed into the mixture. After the addition was complete, the mixture was heated at $140{ }^{\circ} \mathrm{C}$ for another $8 \mathrm{~h}$. After cooling down to room temperature, the mixture was poured into $200 \mathrm{~mL}$ of 1 $\mathrm{M} \mathrm{NaOH}$ and extracted with hexanes $(300 \mathrm{~mL} \times 3)$ The combined extract was washed with $1 \mathrm{M} \mathrm{NaOH}$, dried with $\mathrm{MgSO}_{4}$ and rotary evaporated. The resulting brown oil was loaded onto a short silica gel column (6 $\mathrm{cm} \mathrm{H} \times 10 \mathrm{~cm} \varnothing$ ) and eluted with ca. $1 \mathrm{~L}$ of hexanes. The pure chromatographic fractions were rotary evaporated to dryness to afford the product as a white crystalline solid. If necessary, the product can be recrystallized from hexanes. (6.3 g, $48 \%$ yield based on acetone). ${ }^{1} \mathbf{H} \mathbf{~ N M R}\left(300 \mathrm{MHz}, \mathrm{CDCl}_{3}\right) \delta 1.62,1.63$, $2.34,6.91,6.94,6.97,6.98,7.01,7.19,7.19,7.19 .{ }^{13} \mathbf{C ~ N M R}\left(75 \mathrm{MHz}, \mathrm{CDCl}_{3}\right) \delta 20.96,32.39,33.91,77.00$, 115.98, 126.43, 127.91, 129.62, 131.94, 148.35.; IR $\left(\mathrm{cm}^{-1}\right): 2966,1489,1362,1292,116,1167,1132,1087$, 1024, 1000, 941, 898, 809; HRMS (ESI-TOF) m/z: [M+ H] ${ }^{+}$calcd for $\mathrm{C}_{17} \mathrm{H}_{19} \mathrm{O}, 239.1430$; found 239.1456. mp: $138-140{ }^{\circ} \mathrm{C}$;<smiles>Cc1ccc2c(c1)C(C)(C)c1cc(C)ccc1O2</smiles>

4b. 4,5-dibromo-2,7,9,9-tetramethyl-9H-xanthene (S-5). A solution of S-4 (8.167 g, $34 \mathrm{mmol})$ in 100 $\mathrm{mL} \mathrm{CH} \mathrm{Cl}_{2}$ stirring at $0{ }^{\circ} \mathrm{C}$ was treated with bromine $(5.5 \mathrm{~mL}, 106 \mathrm{mmol})$ added using a syringe pump over the course of $2 \mathrm{~h}$ and then allowed to warm up to room temperature. After complete conversion by TLC (another $2 \mathrm{~h}$ ), the mixture was quenched with $100 \mathrm{~mL}$ of $10 \% \mathrm{Na}_{2} \mathrm{SO}_{3}$ and extracted with $\mathrm{CH}_{2} \mathrm{Cl}_{2}(100$ $\mathrm{mL} \times 3$ ). The combined organic mixture was washed with brine, dried over $\mathrm{MgSO}_{4}$, and rotary evaporated to dryness. The resulting yellow oil was eluted with hexanes through a short silica gel column $(6 \mathrm{~cm} \mathrm{H} \times$ 
$10 \mathrm{~cm} \mathrm{Ø).} \mathrm{Evaporation} \mathrm{gave} \mathrm{the} \mathrm{product} \mathrm{as} \mathrm{a} \mathrm{white} \mathrm{solid} \mathrm{(10.2} \mathrm{g,} \mathrm{76 \%} \mathrm{yield).}{ }^{1} \mathbf{H}$ NMR $\left(300 \mathrm{MHz}, \mathrm{CDCl}_{3}\right)$ $\delta 1.59(\mathrm{~s}, 6 \mathrm{H}), 2.32(\mathrm{~s}, 6 \mathrm{H}), 7.11-7.12(\mathrm{~m}, 2 \mathrm{H}), 7.29-7.30(\mathrm{~m}, 2 \mathrm{H}) .{ }^{13} \mathrm{C} \mathrm{NMR}\left(75 \mathrm{MHz}, \mathrm{CDCl}_{3}\right) \delta 20.74$, 31.84, 35.29, 110.48, 125.34, 131.38, 131.67, 131.69, 133.72, 145.30. IR $\left(\mathrm{cm}^{-1}\right): 2953,1441,1296,1257$, 1096, 901, 847, 789, 734, 556; HRMS (ESI-TOF) m/z: calcd for $\left[\mathrm{C}_{17} \mathrm{H}_{16} \mathrm{Br}_{2} \mathrm{O}+\mathrm{nNa}\right]^{+\mathrm{n}}, 418.9617$; found, 418.9433. mp: $158-160{ }^{\circ} \mathrm{C}$.<smiles>Cc1cc(Br)c2c(c1)C(C)(C)c1cc(C)cc(Br)c1O2</smiles><smiles>Cc1cc(C)c(Oc2c(NC(=O)O)cc(C)cc2C(C)(C)C)c(NC=O)c1</smiles>

4c. $\mathbf{N}, \mathbf{N}^{\prime}-\left(\mathbf{2 , 7 , 9 , 9 - t e t r a m e t h y l - 9 H - x a n t h e n e - 4 , 5 - d i y l ) d i f o r m a m i d e ~ ( S - 6 ) . ~}{ }^{\mathbf{5}}\right.$ A mixture of S-5 (1.18 g, 3.2 mmol), anhydrous $\mathrm{CuSO}_{4}(1.2 \mathrm{~g}, 7.5 \mathrm{mmol}), \mathrm{K}_{2} \mathrm{CO}_{3} \cdot 1.5 \mathrm{H}_{2} \mathrm{O}(12.5 \mathrm{mmol})$ and formamide $(30 \mathrm{~mL})$ was stirred at $160^{\circ} \mathrm{C}$ for $8 \mathrm{~h}$ in a $100 \mathrm{~mL}$ round bottom flask equipped with a reflux condenser. After cooling to room temperature, the reaction mixture was poured into saturated aqueous $\mathrm{NH}_{4} \mathrm{Cl}(100 \mathrm{~mL})$ and $\mathrm{CH}_{2} \mathrm{Cl}_{2}$ $(150 \mathrm{~mL})$. The entire mixture (including the aqueous phase) was filtered through Celite. The Celite cake was rinsed with THF $(200 \mathrm{~mL})$. The combined filtrate was washed with saturated aqueous $\mathrm{NH}_{4} \mathrm{Cl}$ and extracted with $\mathrm{CH}_{2} \mathrm{Cl}_{2}(200 \mathrm{~mL} \times 3)$. The organic phase was dried with $\mathrm{Na}_{2} \mathrm{SO}_{4}$ and rotary evaporated. The residue was purified by flash chromatography (hexane/acetone 2:1) to afford the product as a white solid (768 mg, 74\% yield). ${ }^{1} \mathbf{H}$ NMR $\left(300 \mathrm{MHz}, \mathrm{CDCl}_{3}\right) \delta 1.62(\mathrm{~s}, 6 \mathrm{H}), 1.63(\mathrm{~s}, 3 \mathrm{H}), 2.36(\mathrm{~s}, 6 \mathrm{H}), 3.36(\mathrm{~s}, 3 \mathrm{H})$, $6.94-7.07(\mathrm{~m}, 5 \mathrm{H}$, aromatic), 8.17-8.18 (d, $1 \mathrm{H}, \mathrm{J}=3 \mathrm{~Hz}$, aromatic), $8.54-8.55(\mathrm{~d}, 1 \mathrm{H}, \mathrm{J}=3 \mathrm{~Hz}$, aromatic), $8.83-8.97$ (m, 2H, -NH), 9.78 (s, 1H, -NH), 10.58 - 10.61 (d, 1H, J = 9 Hz, -CHO), 10.85 - 10.89 (d, 1H, $\mathrm{J}=9 \mathrm{~Hz},-\mathrm{CHO}) .{ }^{13} \mathbf{C}$ NMR $\left(75 \mathrm{MHz}, \mathrm{CDCl}_{3}\right): \delta 21.15,21.17,21.36,32.42,32.58,34.16,116.52,116.83$, $119.96,121.44,122.69,123.40,124.52,125.32$, 125.34, 129.12, 130.56, 131.08, 132.91, 132.94, 132.96, $136.95,137.93,138.21,159.88,164.01,165.06$. IR $\left(\mathrm{cm}^{-1}\right): 1675,1614,1536,1416,1289,1226,1173,854$ 718, 622, 579, 509; HRMS (ESI-TOF) m/z: calcd for $\left[\mathrm{C}_{19} \mathrm{H}_{20} \mathrm{~N}_{2} \mathrm{O}_{3}+\mathrm{Na}\right]^{+}, 347.1366$; found, 347.1362 . mp: $235-237^{\circ} \mathrm{C}(\mathrm{dec})$.<smiles>Cc1cc(N)c2c(c1)Oc1c(N)cc(C)cc1C(C)(C)c1cc(C)cc(NC=O)c1O2</smiles>

4d. 2,7,9,9-tetramethyl-9H-xanthene-4,5-diamine (9). A mixture of S-6 (507 mg, $1.56 \mathrm{mmol}$ ) and EtOH $(3 \mathrm{~mL})$ was treated with aqueous $\mathrm{NaOH}\left(500 \mathrm{mg}, 12.5 \mathrm{mmol}\right.$ in $\left.1 \mathrm{~mL} \mathrm{H}_{2} \mathrm{O}\right)$ and refluxed for $1 \mathrm{~h}$, at which time TLC analysis indicated full conversion. After cooling to room temperature, the mixture was diluted with EtOAc $(20 \mathrm{~mL})$, poured into brine, and extracted with EtOAc $(20 \mathrm{~mL} \times 3)$. The combined organic phase was washed with brine, dried with $\mathrm{Na}_{2} \mathrm{SO}_{4}$ and rotary evaporated to dryness. The residue was recrystallized from hexane to afford the product as a white powder (376.5 $\mathrm{mg}, 90 \%$ yield). ${ }^{1} \mathbf{H}$ NMR (300 $\left.\mathrm{MHz}, \mathrm{CDCl}_{3}\right) \delta 1.58(\mathrm{~s}, 6 \mathrm{H}), 2.26(\mathrm{~s}, 6 \mathrm{H}), 3.64(\mathrm{br} \mathrm{s}, 4 \mathrm{H}), 6.48(\mathrm{~s}, 2 \mathrm{H}), 6.62(\mathrm{~s}, 2 \mathrm{H}) .{ }^{13} \mathrm{C} \mathrm{NMR}(151 \mathrm{MHz}$, $\left.\mathrm{CDCl}_{3}\right) \delta 21.08,21.11,31.88,34.05,114.26,116.00,129.92,131.93,133.95,136.48$. IR (cm-1):1625, 1476, 1273, 1212, 1165, 1031, 831, 554, HRMS (ESI-TOF) $\mathrm{m} / \mathrm{z}$ : calcd for $\left[\mathrm{C}_{17} \mathrm{H}_{20} \mathrm{~N}_{2} \mathrm{O}+\mathrm{H}\right]^{+}, 269.1648$; found, 269.1643. mp: $50^{\circ} \mathrm{C}$. 


\section{SYNTHESIS AND REDUCTION OF MODEL COMPOUND 12.}<smiles></smiles>

5a. N1 ,N6-bis(5-amino-2,7,9,9-tetramethyl-9H-xanthen-4-yl)phenazine-1,6-dicarboxamide (10). To a solution of $9(1.94 \mathrm{~g}, 5.2 \mathrm{mmol})$ in $5 \mathrm{~mL}$ MeCN stirring at room temperature was slowly added a suspension of $4(1.81 \mathrm{~g}, 2.6 \mathrm{mmol})$ in $3 \mathrm{~mL} \mathrm{MeCN}$. Orange-red precipitate was observed almost immediately. After stirring for $12 \mathrm{~h}$, the precipitate was separated by centrifugation, washed repeatedly with $\mathrm{MeCN}(7 \times 5 \mathrm{~mL})$, and dried under vacuum. The product thus obtained was essentially pure $\left(1.62 \mathrm{~g}, 81 \%\right.$ yield). ${ }^{1} \mathbf{H}$ NMR $\left(600 \mathrm{MHz}, \mathrm{CDCl}_{3}\right) \delta 1.70(\mathrm{~s}, 12 \mathrm{H}), 2.28(\mathrm{~s}, 6 \mathrm{H}), 2.44(\mathrm{~s}, 6 \mathrm{H}), 6.40(\mathrm{~s}, 2 \mathrm{H}), 6.68(\mathrm{~s}, 2 \mathrm{H}), 7.09(\mathrm{~s}, 2 \mathrm{H}), 7.26$ $(\mathrm{s}, 2 \mathrm{H}), 8.08-8.10(\mathrm{t}, 2 \mathrm{H}, \mathrm{J}=6 \mathrm{~Hz}), 8.14(\mathrm{~s}, 2 \mathrm{H}), 8.67-8.69(\mathrm{~d}, 2 \mathrm{H}, \mathrm{J}=6 \mathrm{~Hz}), 9.19-9.20(\mathrm{~d}, 2 \mathrm{H}, \mathrm{J}=6 \mathrm{~Hz})$, $12.96(\mathrm{~s}, 2 \mathrm{H}),{ }^{13} \mathrm{C}$ NMR $\left(151 \mathrm{MHz}, \mathrm{CDCl}_{3}\right) \delta 21.20,21.48,31.52,34.62,114.08,115.47,121.55,122.14$, $125.64,129.57,130.63,130.85,131.43,132.31,132.88,134.10,134.18,136.62,137.01,140.09,140.56$, 141.43, 162.14. IR $\left(\mathrm{cm}^{-1}\right): 1734,1540,1473,1363,748$; HRMS (APCI-TOF) m/z: calcd for $\left[\mathrm{C}_{48} \mathrm{H}_{44} \mathrm{~N}_{6} \mathrm{O}_{4}\right.$ $+\mathrm{H}]^{+}:$769.3497, found: 769.3646 . mp: $>300^{\circ} \mathrm{C}$.

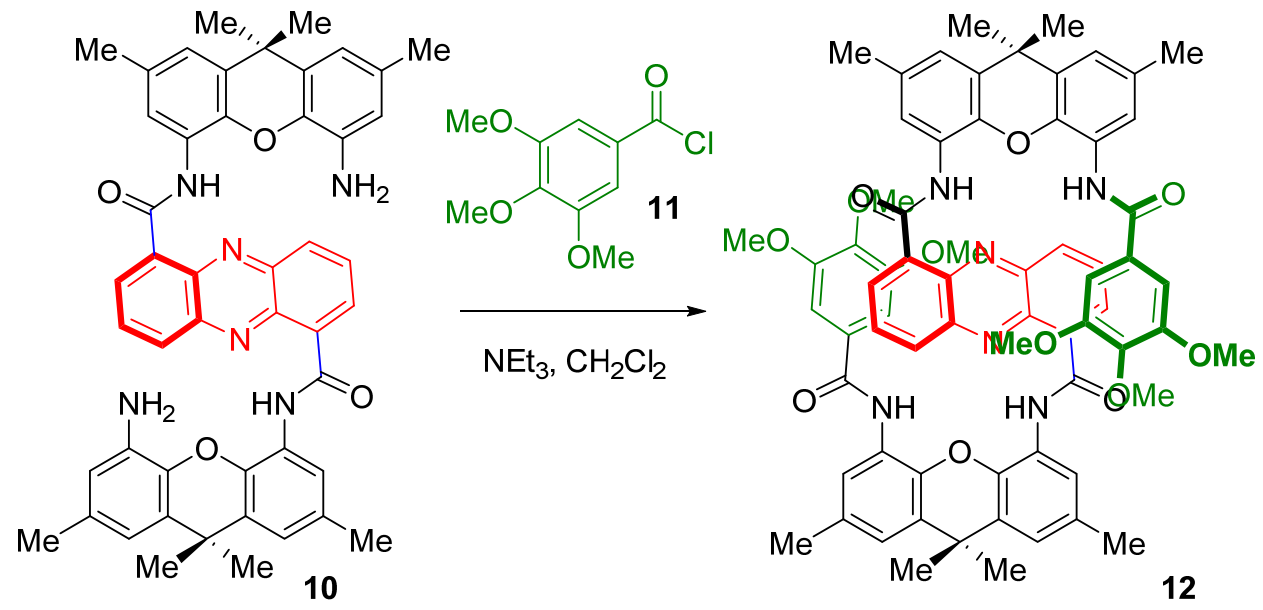

5b. N1,N6-bis(2,7,9,9-tetramethyl-5-(3,4,5-trimethoxybenzamido)-9H-xanthen-4-yl)phenazine-1,6dicarboxamide (12). A suspension of $10(116 \mathrm{mg}, 0.15 \mathrm{mmol})$ in $\mathrm{CH}_{2} \mathrm{Cl}_{2}$ was treated successively with $\mathrm{NEt}_{3}(45 \mu \mathrm{L}, 0.33 \mathrm{mmol})$ and 3,4,5-trimethoxybenzoyl chloride $11(77 \mathrm{mg}, 0.33 \mathrm{mmol})$ added slowly at room temperature. TLC indicated complete conversion in $2 \mathrm{~h}$. The mixture was quenched with saturated aqueous $\mathrm{K}_{2} \mathrm{CO}_{3}$ and extracted with $\mathrm{CH}_{2} \mathrm{Cl}_{2}$. The organic phase was rotary evaporated. A mixture of the crude product with $80 \mathrm{~mL}$ of saturated aqueous $\mathrm{K}_{2} \mathrm{CO}_{3}$ was refluxed for $2 \mathrm{~h}$, allowed to cooling down to room temperature overnight, and then extracted with $100 \mathrm{~mL}$ of $\mathrm{CH}_{2} \mathrm{Cl}_{2}$. The organic extract was dried over 
$\mathrm{MgSO}_{4}$ and rotary evaporated. The residue was recrystallized from acetone to afford essentially pure 12 (107mg, 62\% yield). ${ }^{1} \mathbf{H}$ NMR $\left(300 \mathrm{MHz}, \mathrm{CD}_{2} \mathrm{Cl}_{2}\right) \delta 1.75(\mathrm{~s}, 12 \mathrm{H}), 2.41(\mathrm{~s}, 6 \mathrm{H}), 2.53(\mathrm{~s}, 6 \mathrm{H}), 3.25(\mathrm{~s}, 12 \mathrm{H})$, 3.45 (s, 6H), 5.84 (s, 4H), 7.14 (s, 2H), 7.20 (s, 2H), 8.01 - 8.06 (t, 2H, J=6 Hz), 8.13 (s, 2H), 8.26-8.28 (d, $2 \mathrm{H}, \mathrm{J}=6 \mathrm{~Hz}), 8.33(\mathrm{~s}, 2 \mathrm{H}),, 9.13-9.16(\mathrm{~d}, 2 \mathrm{H}, \mathrm{J}=6 \mathrm{~Hz}), 13.21(\mathrm{~s}, 2 \mathrm{H}) .{ }^{13} \mathbf{C}$ NMR $\left(126 \mathrm{MHz}, \mathrm{CD}_{2} \mathrm{Cl}_{2}\right) \delta$ $21.83,21.97,31.14,31.62,35.33,56.08,60.14,102.74,119.74,121.46,121.69,122.38,126.52,126.66$, $128.98,129.50,131.24,131.65,132.10,133.68,133.87,134.06,137.12,138.82,139.97,140.07,140.16$, 140.99, 152.32, 161.98, 164.54, 206.91. IR $\left(\mathrm{cm}^{-1}\right): 3423,1556,1501,1419,1333,1211,1126,855,749$, 569. HRMS (APCI-TOF) m/z: calcd for $\left[\mathrm{C}_{68} \mathrm{H}_{64} \mathrm{~N}_{6} \mathrm{O}_{12}+\mathrm{H}\right]^{+}: 1157.4655$, found $1158.4810 . \mathbf{m p :}>300{ }^{\circ} \mathrm{C}$.

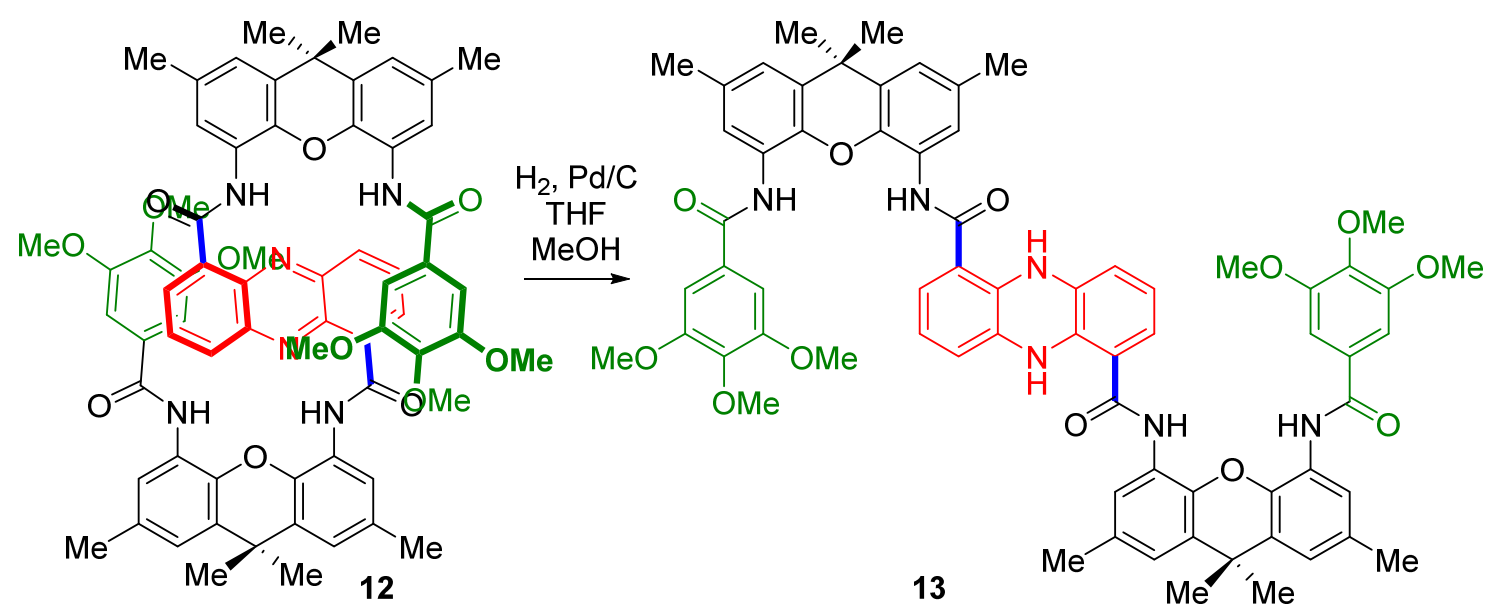

5c. N1,N6-bis(2,7,9,9-tetramethyl-5-(3,4,5-trimethoxybenzamido)-9H-xanthen-4-yl)-5,10-dihydrophenazine-1,6-dicarboxamide (13). Compound $12(30 \mathrm{mg}, 0.026 \mathrm{mmol})$ was hydrogenated using $10 \mathrm{wt} \%$ $\mathrm{Pd} / \mathrm{C}(5 \mathrm{mg}, 0.005 \mathrm{mmol}), 0.7 \mathrm{~mL}$ of THF and $0.7 \mathrm{~mL}$ of $\mathrm{MeOH}$ at $20 \mathrm{psi}$ for $18 \mathrm{~h}$ as described above for 6. The solution of the product in $\mathrm{CDCl}_{3}$ was stable when kept in an NMR tube sealed under argon. Upon deliberate exposure to air, however, it underwent over $90 \%$ oxidation within 20 min. ${ }^{1} \mathbf{H}$ NMR $(600 \mathrm{MHz}$, $\left.\mathrm{CDCl}_{3}\right) \delta 1.64(\mathrm{~s}, 12 \mathrm{H}), 2.37(\mathrm{~s}, 6 \mathrm{H}), 2.39(\mathrm{~s}, 6 \mathrm{H}), 3.83(\mathrm{~s}, 12 \mathrm{H}), 3.88(\mathrm{~s}, 6 \mathrm{H}), 5.47-5.48(\mathrm{t}, 2 \mathrm{H}, \mathrm{J}=6 \mathrm{~Hz})$, $5.83-5.84(\mathrm{~d}, 2 \mathrm{H}, \mathrm{J}=6 \mathrm{~Hz}), 6.26-6.27(\mathrm{t}, 2 \mathrm{H}, \mathrm{J}=6 \mathrm{~Hz}), 7.02(\mathrm{~s}, 4 \mathrm{H}), 7.06(\mathrm{~m}, 4 \mathrm{H}), 7.67$ - $7.69(\mathrm{~m}, 4 \mathrm{H})$, $7.93(\mathrm{~s}, 2 \mathrm{H}), 8.03(\mathrm{~s}, 2 \mathrm{H}), 8.88(\mathrm{~s}, 2 \mathrm{H}) .{ }^{13} \mathbf{C} \mathbf{N M R}\left(151 \mathrm{MHz}, \mathrm{CDCl}_{3}\right) \delta$ 21.31, 21.40, 31.87, 34.50, 56.17, 60.81, 104.38, 111.63, 114.14 - 114.23 (d, ), 117.51, 119.66, $121.44-121.87$ (dd, J = 20 Hz ), 122.53 $122.73\left(\mathrm{dd}, \mathrm{J}_{1}=24 \mathrm{~Hz}, \mathrm{~J}_{2}=6 \mathrm{~Hz}\right) 124.57-124.94\left(\mathrm{dd}, \mathrm{J}_{1}=47 \mathrm{~Hz}, \mathrm{~J}_{2}=4 \mathrm{~Hz}\right), 128.79,129.97,130.47$, $130.61,130.87,132.83-132.87(\mathrm{~d}, \mathrm{~J}=6 \mathrm{~Hz}), 133.53-133.62(\mathrm{~d}, \mathrm{~J}=13.5 \mathrm{~Hz}), 136.73-136.89$ (d, J = 24 $\mathrm{Hz}), 139.26-139.40\left(\mathrm{~d}, \mathrm{~J}_{1}=9 \mathrm{~Hz}, \mathrm{~J}_{2}=4.5 \mathrm{~Hz}\right), 141.26,153.34,165.71-165.79$ (d, J = $\left.12 \mathrm{~Hz}\right), 166.39$ $166.50\left(\mathrm{dd}, \mathrm{J}_{1}=4.5 \mathrm{~Hz}, \mathrm{~J}_{2}=12 \mathrm{~Hz}\right)$.

\section{CYCLIC VOLTAMMETRY STUDY}

General. Cyclic voltammetric experiments were performed using a BAS 100B Electrochemical Analyzer in a conventional one-compartment three-electrode cell with glassy carbon as the working electrode, a platinum wire auxiliary electrode, and a $\mathrm{Ag} / \mathrm{AgCl}$ reference electrode. The surface of the working electrode was polished with filter paper immediately before use. The cell was flushed with argon before use and the measurements were conducted under an argon atmosphere at room temperature. THF was freshly distilled from sodium/benzophenone. HPLC grade methanol was degassed using the freeze-pump-thaw technique immediately before use. Lithium perchlorate was used as the electrolyte at $0.1 \mathrm{M}$ concentration. Cyclic voltammograms were recorded at $50 \mathrm{mV} / \mathrm{s}$ sweep rate after 4 cycles. Due to the poor solubility of N, N'- 
diarylphenazine-1,6-dicarboxamides such as $\mathbf{6}$ and $\mathbf{1 2}$ in solvents with a suitable electroactive window, the $\mathrm{N}, \mathrm{N}^{\prime}$-dialkyl derivative 1a (see below) was employed at $0.0025 \mathrm{M}$ concentration.

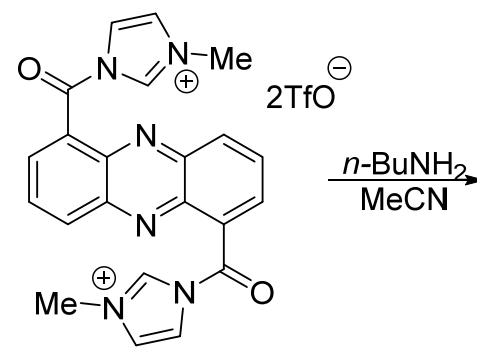

4<smiles>CCCCNC(=O)c1cccc2nc3c(C(=O)NC(C)=O)cccc3nc12</smiles>

$1 \mathrm{a}$

6a. N1,N6-bis-(1-butyl)-phenazine-1,6-dicarboxamide (1a). To a suspension of 4 (697 mg, $1.00 \mathrm{mmol})$ in $5 \mathrm{~mL}$ of MeCN stirring at room temperature was slowly added $n$-butylamine $(0.2 \mathrm{~mL}, 2 \mathrm{mmol})$. TLC indicated complete conversion after $30 \mathrm{~min}$. The reaction mixture was rotary evaporated, the residue taken up in $\mathrm{CH}_{2} \mathrm{Cl}_{2}$, rinsed with water, and dried over $\mathrm{MgSO}_{4}$. Flash chromatography (hexanes/EtOAc 2:1) afforded the product as a yellow solid (353 mg, 93\% yield). ${ }^{1} \mathbf{H}$ NMR $\left(300 \mathrm{MHz}, \mathrm{CD}_{3} \mathrm{OD}\right) \delta 1.05-1.10$ (t, $6 \mathrm{H}, \mathrm{J}=7.5 \mathrm{~Hz}), 1.54-1.66(\mathrm{~m}, 4 \mathrm{H}), 1.74-1.84(\mathrm{~m}, 4 \mathrm{H}), 3.60-3.66(\mathrm{dd}, 4 \mathrm{H}, \mathrm{J}=6 \mathrm{~Hz}), 8.03-8.09(\mathrm{t}, 2 \mathrm{H}$, $\mathrm{J}=7.5 \mathrm{~Hz}), 8.31-8.34(\mathrm{~d}, 2 \mathrm{H}, \mathrm{J}=7.5 \mathrm{~Hz}), 8.73-8.76(\mathrm{~d}, 2 \mathrm{H}, \mathrm{J}=7.5 \mathrm{~Hz}), 10.58(\mathrm{br}, 2 \mathrm{H}),{ }^{13} \mathbf{C} \mathbf{N M R}(126$ $\left.\mathrm{MHz}, \mathrm{CD}_{3} \mathrm{OD}\right) \delta 12.76,20.09,31.17,39.47,129.56,131.08,132.65,134.72,140.03,141.34,165.19$. IR $\left(\mathrm{cm}^{-1}\right): 3280.87,2926.27,2868.67,2147.40,1642.34,1604.83,1546.21,1529.02,1272.74,1029.85,865.24$, 824.85, 751.56, 635.88. HRMS (ESI+) m/z: calcd for $\left[\mathrm{C}_{22} \mathrm{H}_{26} \mathrm{~N}_{4} \mathrm{O}_{2}+\mathrm{H}\right]^{+}: 379.2129$, found 379.2089. mp: > $300{ }^{\circ} \mathrm{C}$

\section{6b. Cyclic voltammetry of 1a under aprotic and protic conditions.}

THF, Scans 5-10

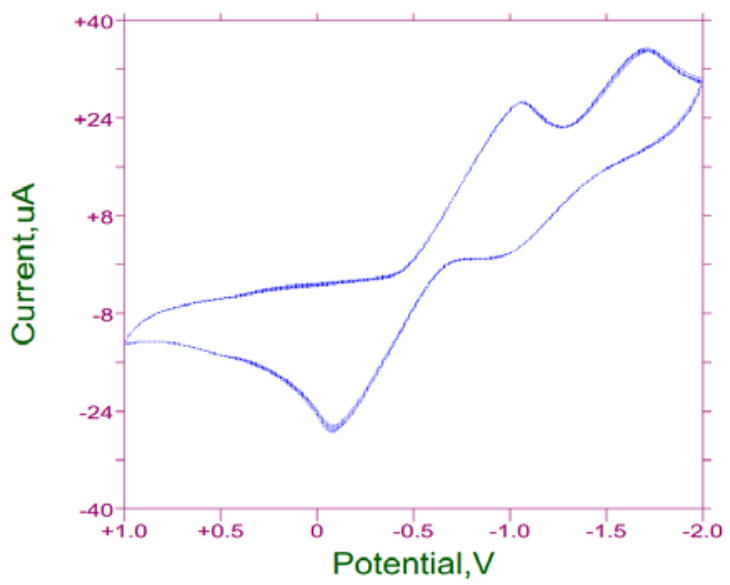

Methanol, Scans 5-20

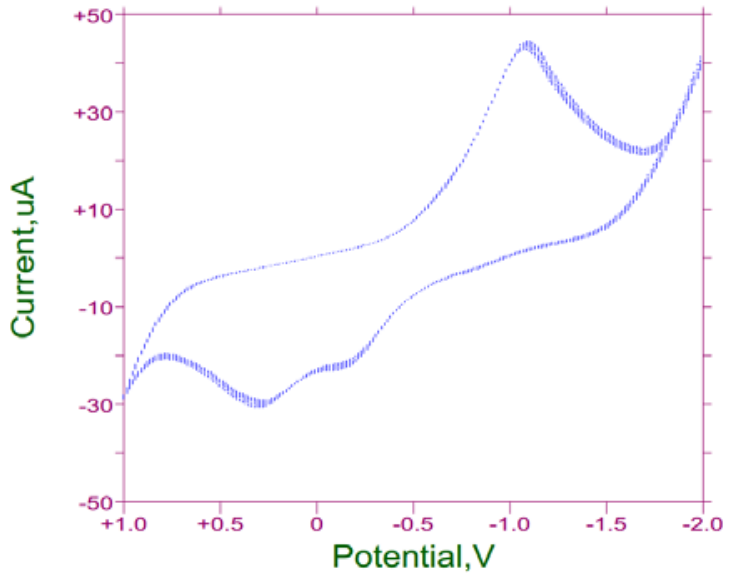

Multiple scans recorded under aprotic and protic conditions indicate the redox stability of 1a. While the $\mathrm{CV}$ in THF contains two distinct reduction waves, only one is observed in methanol.

\section{COMPUTER MODELING.}

7a. Geometry optimization. The conformations of tetramer 8 was optimized by Molecular Mechanics MMFF calculations in gas phase using Spartan'16 software. All other structures shown in Figure S-1 below were obtained by geometry optimization performed at the B3LYP/6-31G* level of theory ${ }^{6}$ in gas phase. 
Geometry optimization of diamine $\mathbf{1 0}$ and its $\mathrm{N}, \mathrm{N}^{\prime}$-diacetyl derivative yielded the corresponding $\mathrm{C}_{2}-$ symmetrical and centrosymmetrical conformers of comparable stability. Model compound 12 yielded only the centrosymmetrical conformer shown during optimization.
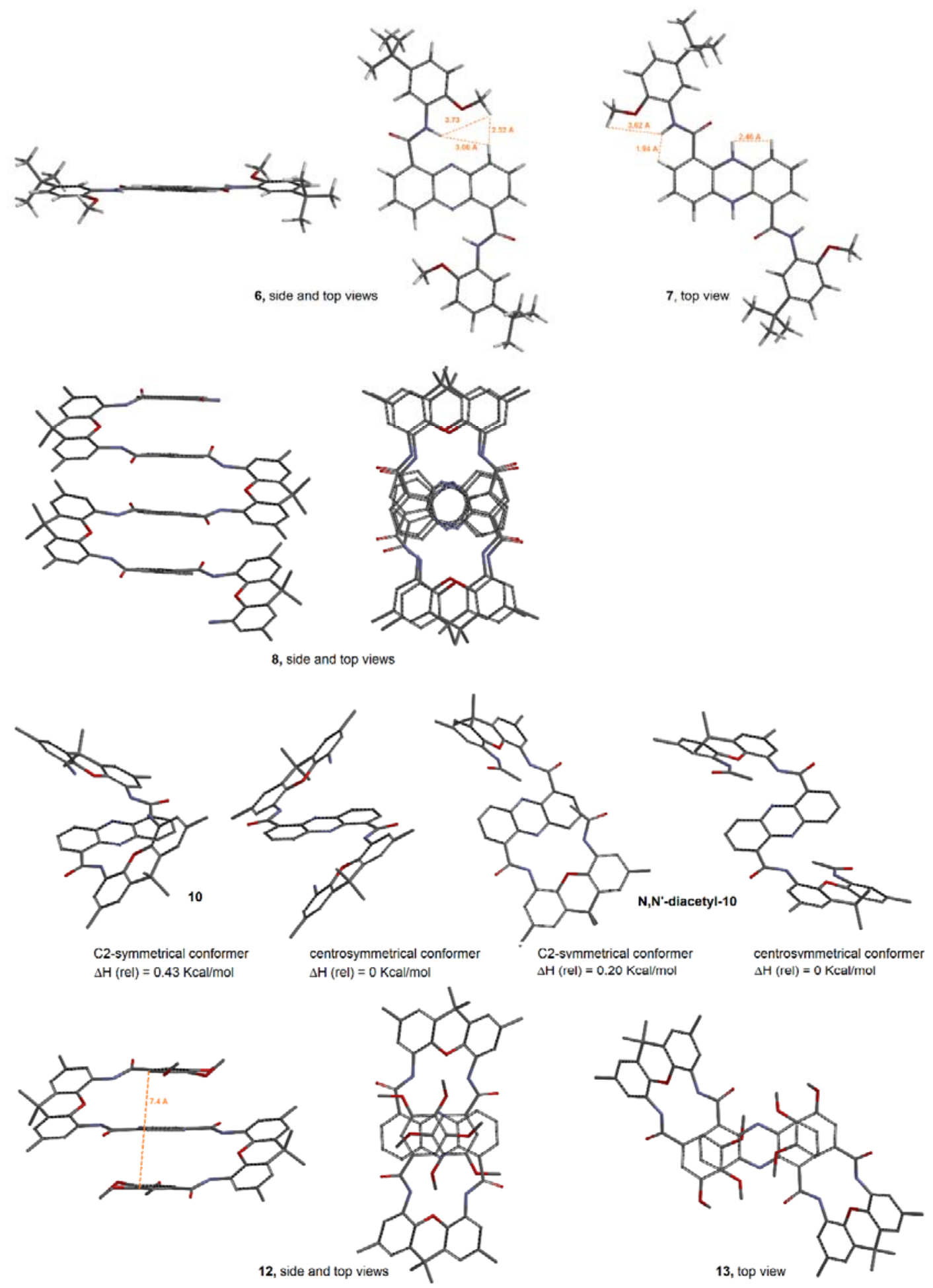

Figure S-1. 
7b. Energy profiles of simple models. The geometries of model phenazine-1,6-dicarboxamide $\mathbf{1 b}$ and its reduced form $\mathbf{2 b}$ were optimized at the B3LYP/6-31G* level of theory in gas phase. The dihedral angles of highlighted bonds in optimized $\mathbf{1 b}$ and $\mathbf{2 b}$ were found to be $173^{\circ}$ and $17^{\circ}$, respectively. The highlighted bonds were then constrained at dihedral angles ranging from $0^{\circ}$ to $360^{\circ}$ at 20 degree intervals and reoptimized. The resulting energy profiles are shown below.<smiles>COc1ccccc1NC(=O)c1cccc2nc3c(C(=O)Nc4ccccc4OC)cccc3nc12</smiles>

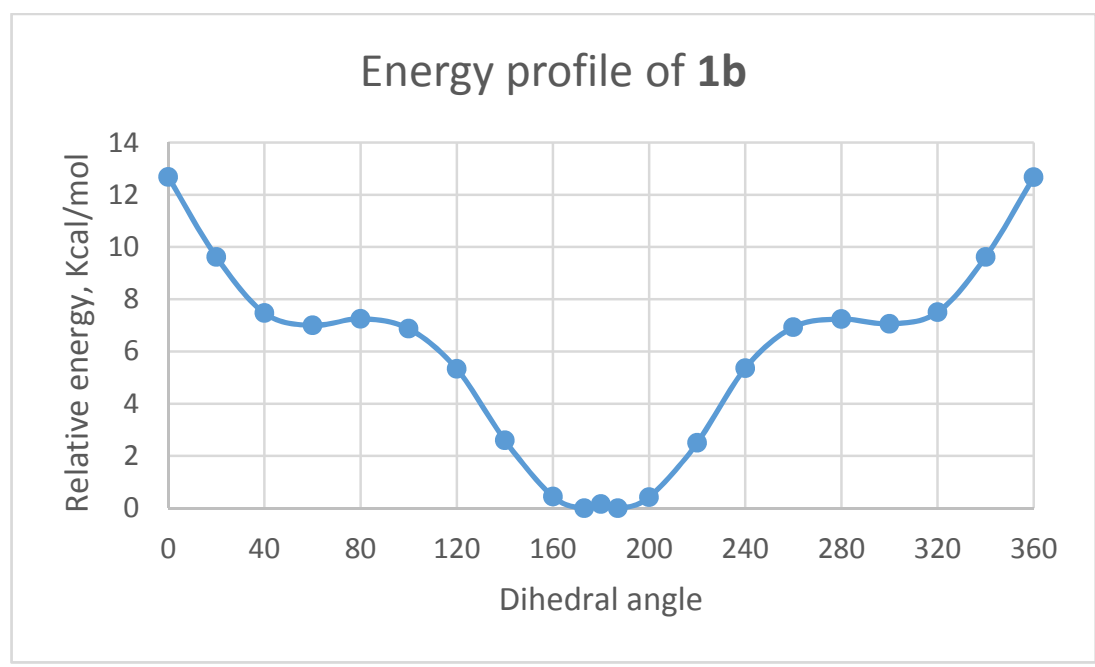<smiles>COc1ccccc1NC(=O)c1cccc2c1Nc1cccc(C(=O)Nc3ccccc3OC)c1N2</smiles>

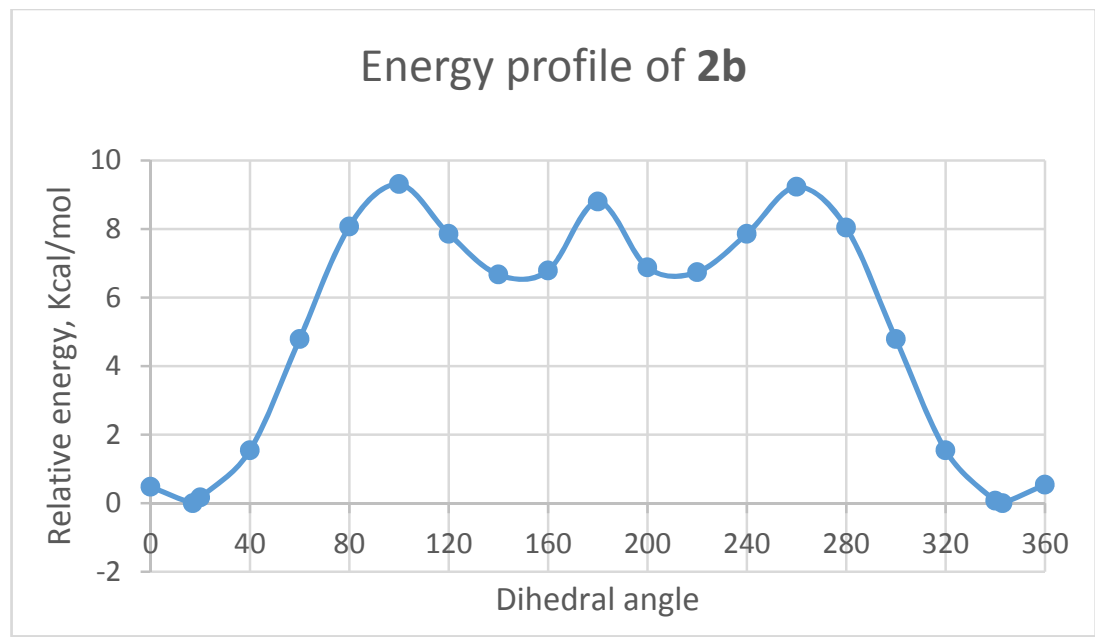

\section{GIBBS FREE ENERGY OF THE DOUBLE FLIP.}

The spectrum of 12 was recorded on a $300 \mathrm{MHz}$ spectrometer in $\mathrm{CD}_{2} \mathrm{Cl}_{2}$ at the temperatures indicated below. At the lowest attainable temperature $\left(-80^{\circ} \mathrm{C}\right)$ the separation between the signals of $\mathbf{h}$ and $\mathbf{h}^{\prime}$ was measured as: $\Delta \delta_{\mathbf{h} / \mathbf{h}^{\prime}}=0.37 \mathrm{ppm}\left(\left|v_{\mathbf{h}}-v_{\mathbf{h}^{\prime}}\right|=111 \mathrm{~Hz}\right)$. At the coalescence temperature $\left(-42{ }^{\circ} \mathrm{C}, \mathrm{T}_{\mathbf{c}}=231\right.$ $\mathrm{K})$, the rate of exchange is: ${ }^{7}$

$\mathrm{k}_{\mathrm{Tc}}=111 \mathrm{~Hz} \times \pi / \sqrt{ } 2=246 \mathrm{~s}^{-1}$

From the Eyring-Polanyi equation, $\Delta \mathrm{G}^{\neq}=\mathrm{R} \times \mathrm{T}_{\mathrm{c}} \times \ln \left[\left(\mathrm{k}_{\mathrm{B} \times \mathrm{T}}\right) /\left(\mathrm{h} \times \mathrm{k}_{\mathrm{Tc}}\right)\right]=$

$=8.314 \mathrm{~J} \cdot \mathrm{mol}^{-1} \cdot \mathrm{K}^{-1} \times 231 \mathrm{~K} \times \ln \left[\left(1.3810^{-23} \mathrm{~J} \cdot \mathrm{K}^{-1} \times 231 \mathrm{~K}\right) /\left(6.6310^{-34} \mathrm{~J} \cdot \mathrm{s} \times 246 \mathrm{~s}^{-1}\right)\right]=$

$=45,516 \mathrm{~J} / \mathrm{mol}=\mathbf{1 0 . 9} \mathrm{Kcal} / \mathbf{m o l}$ 
9. 1D NMR SPECTRA
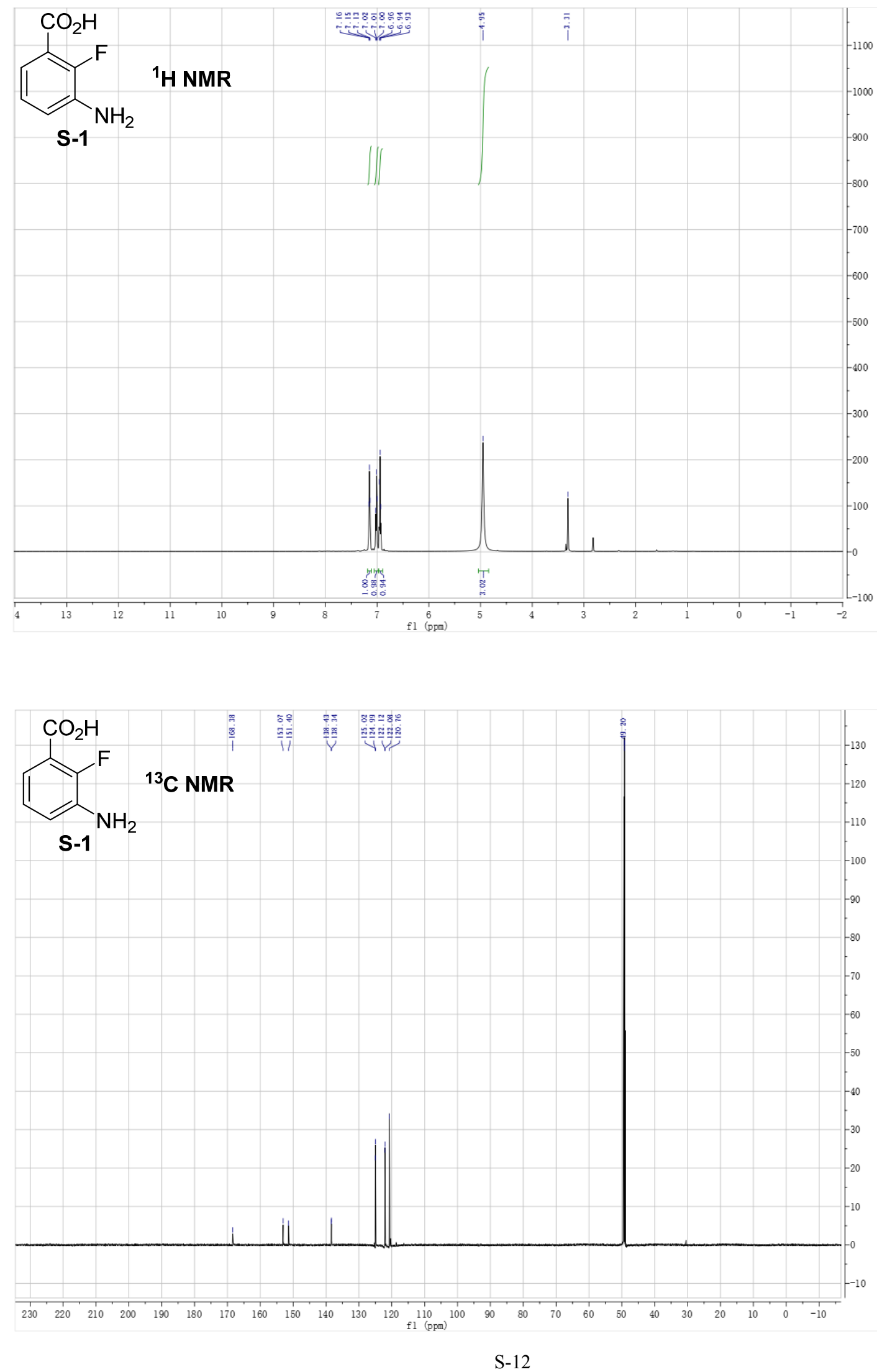

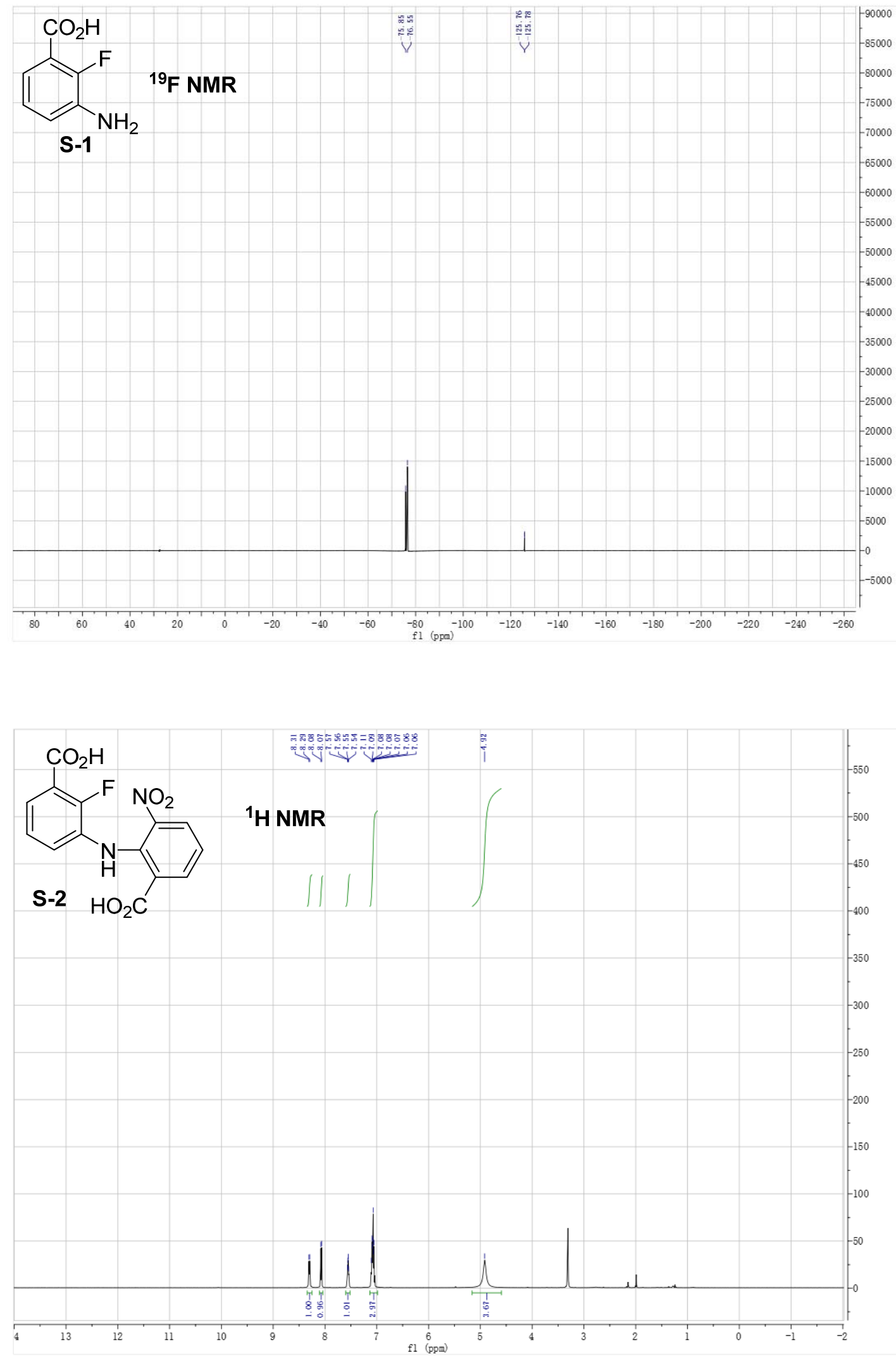

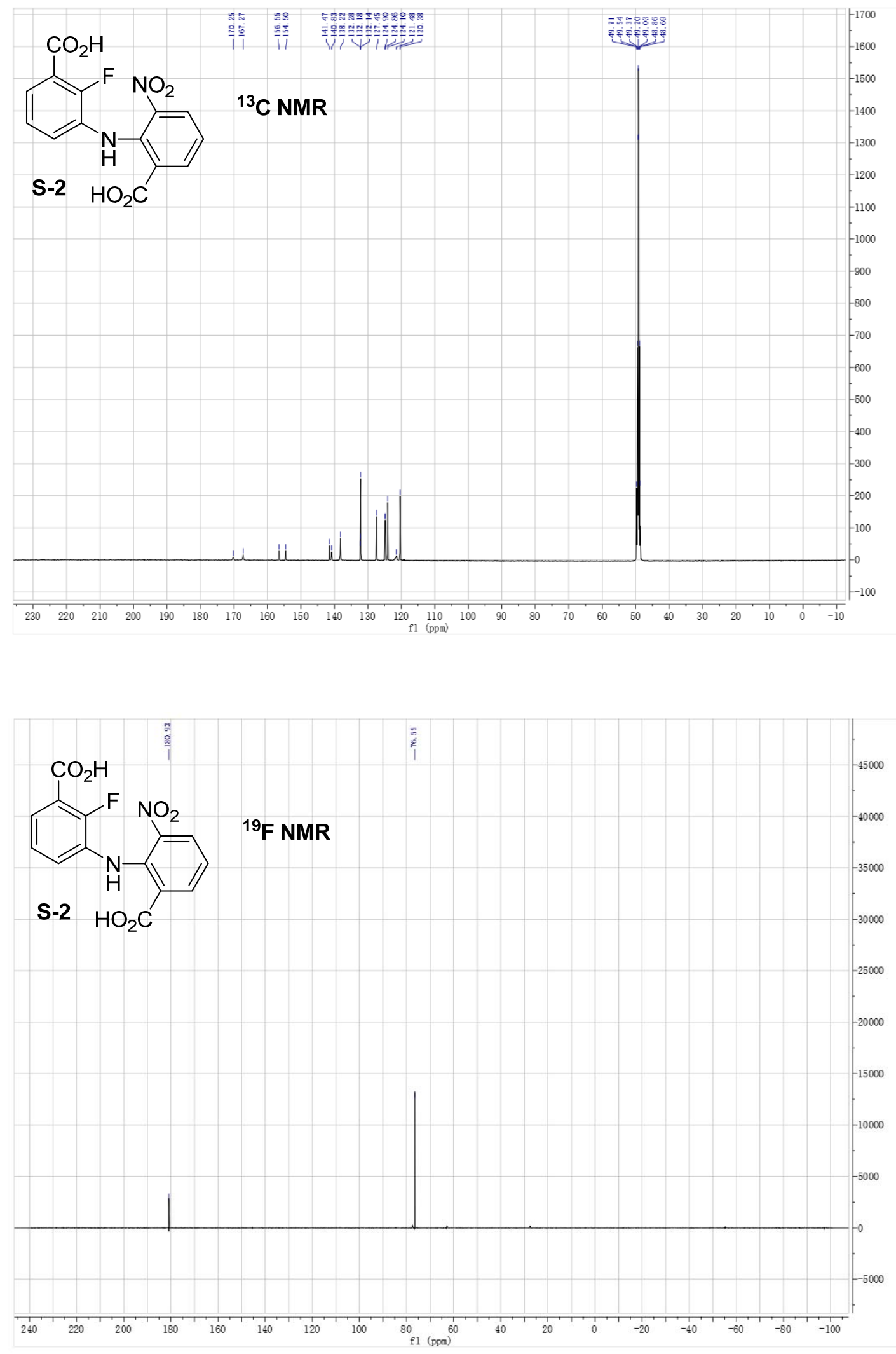

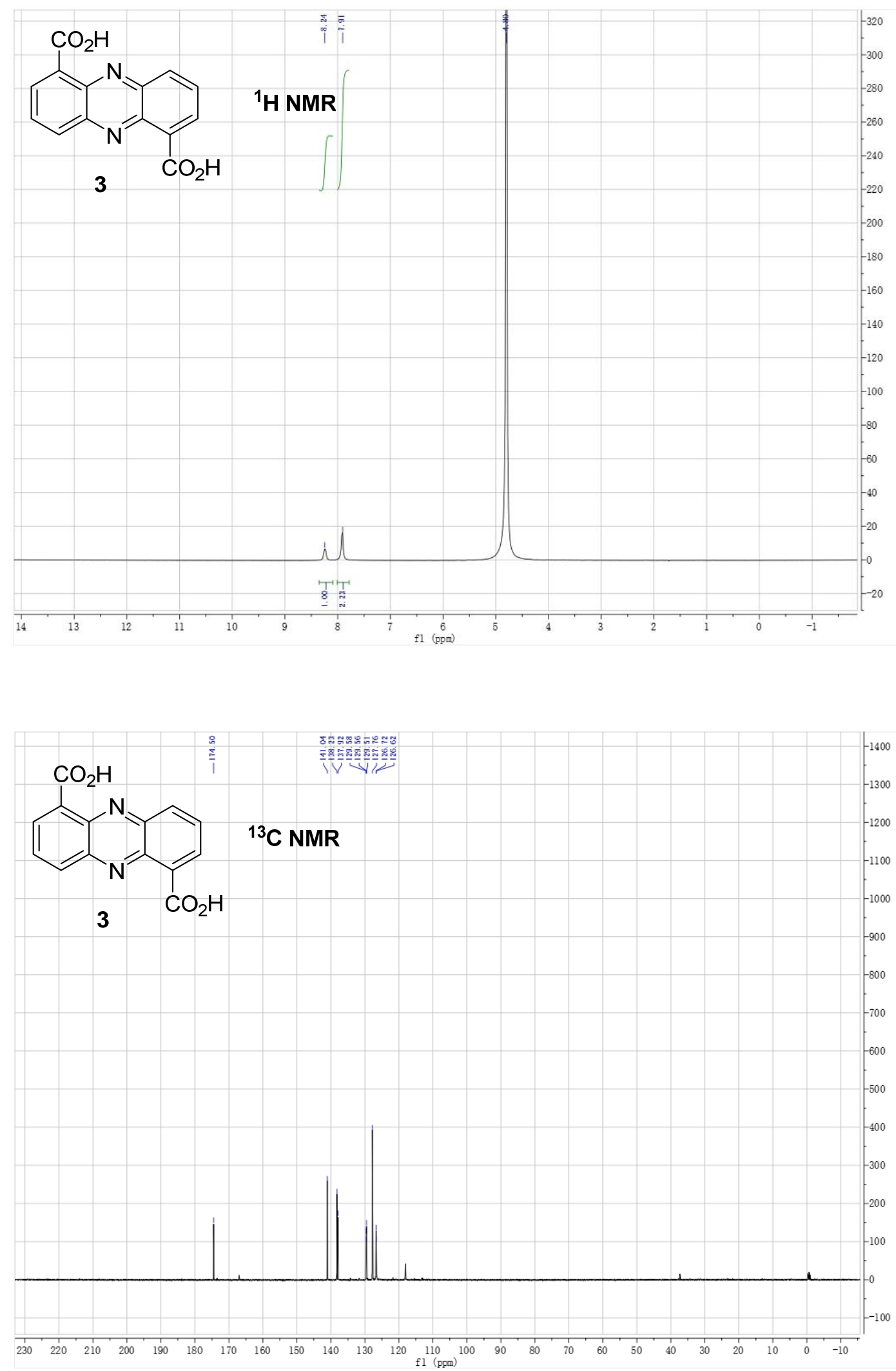

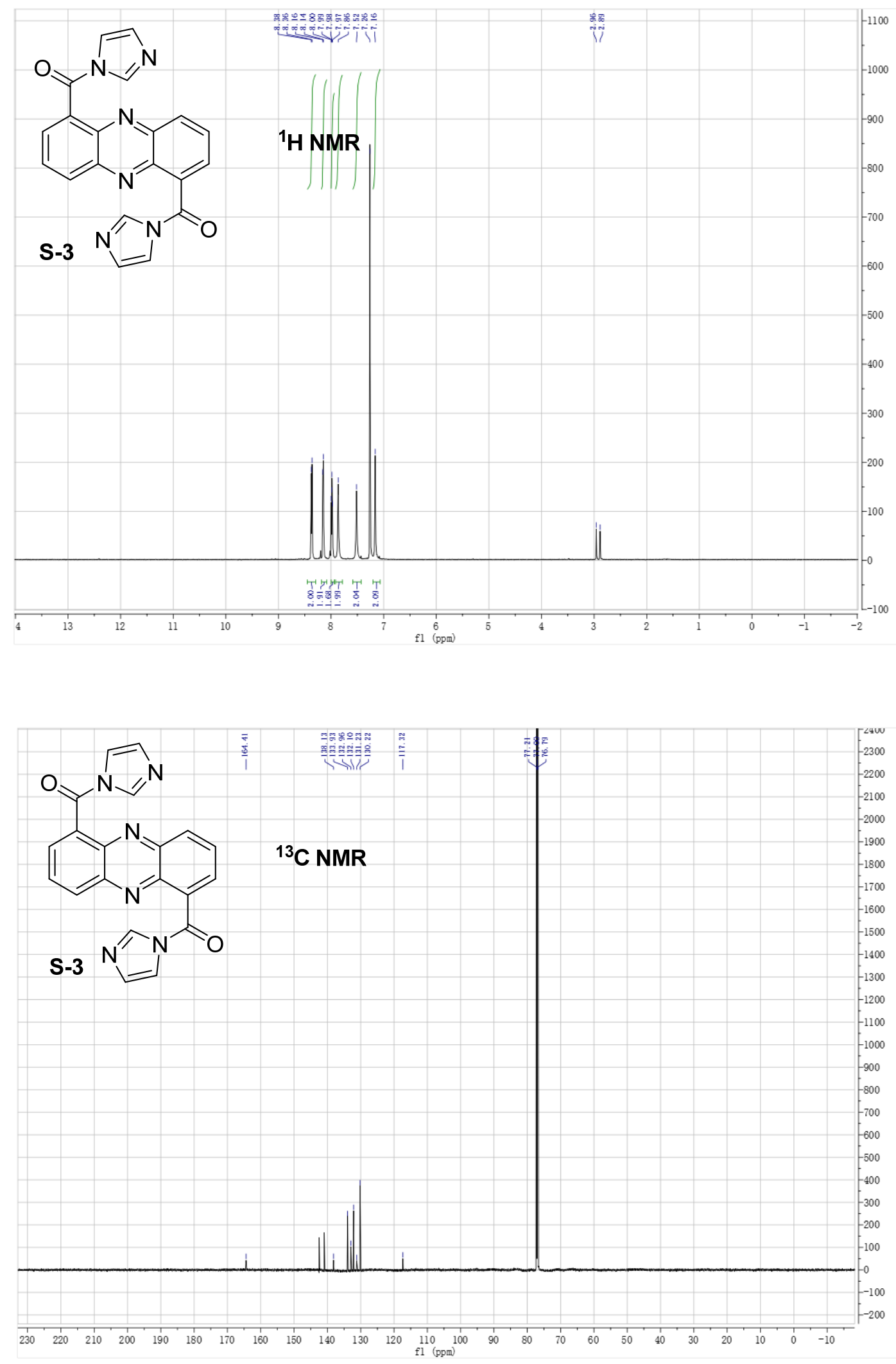

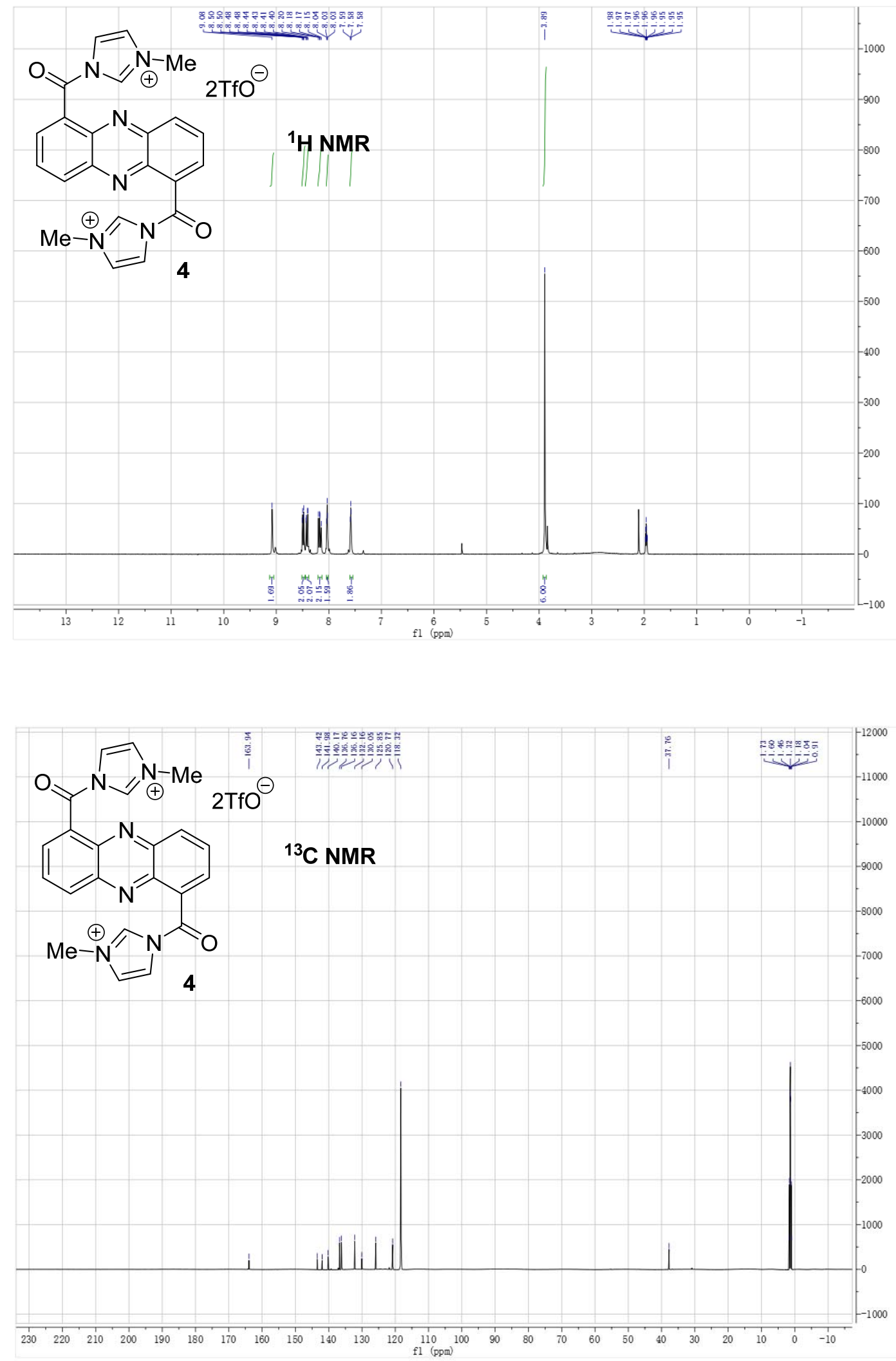

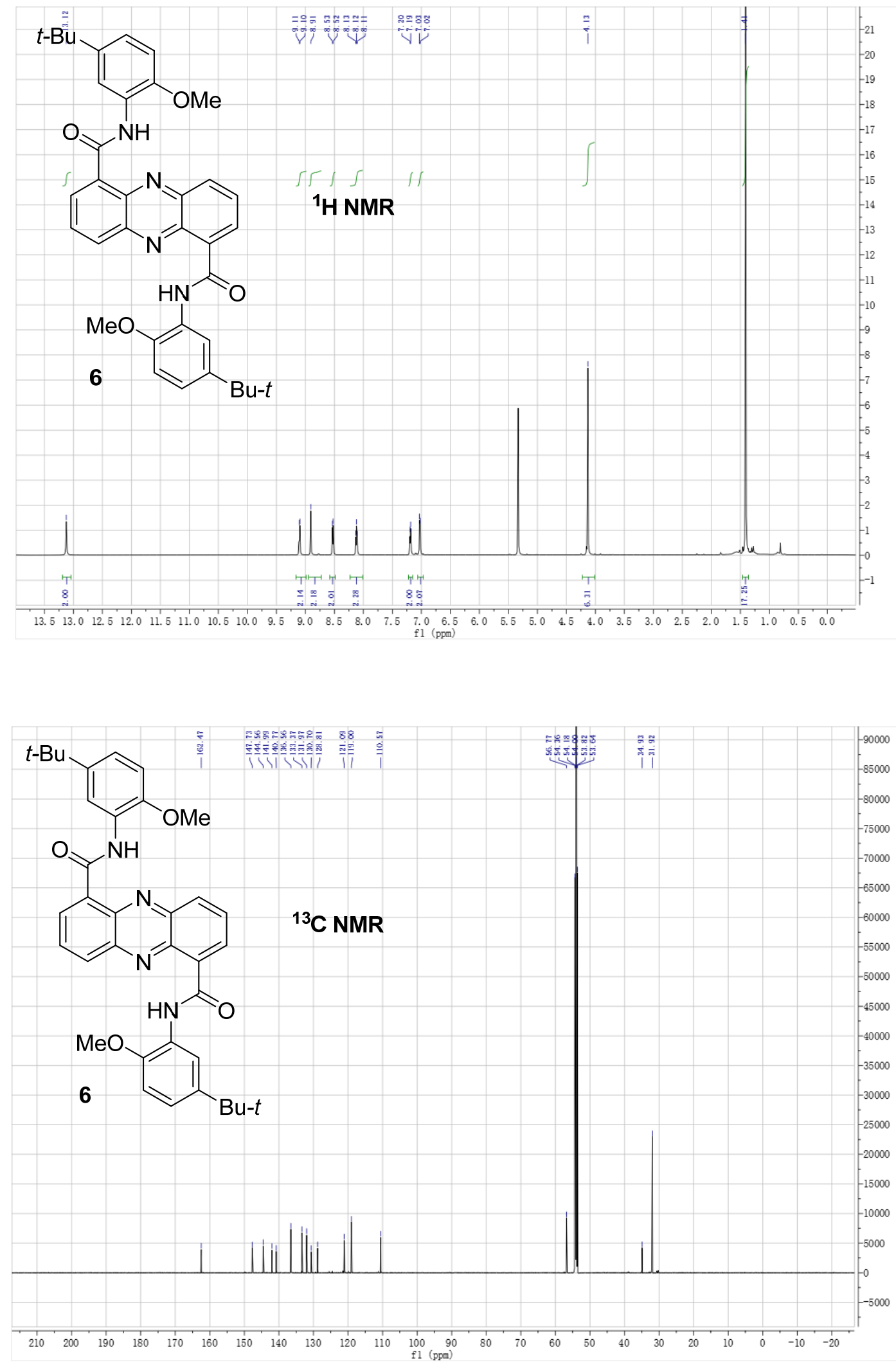

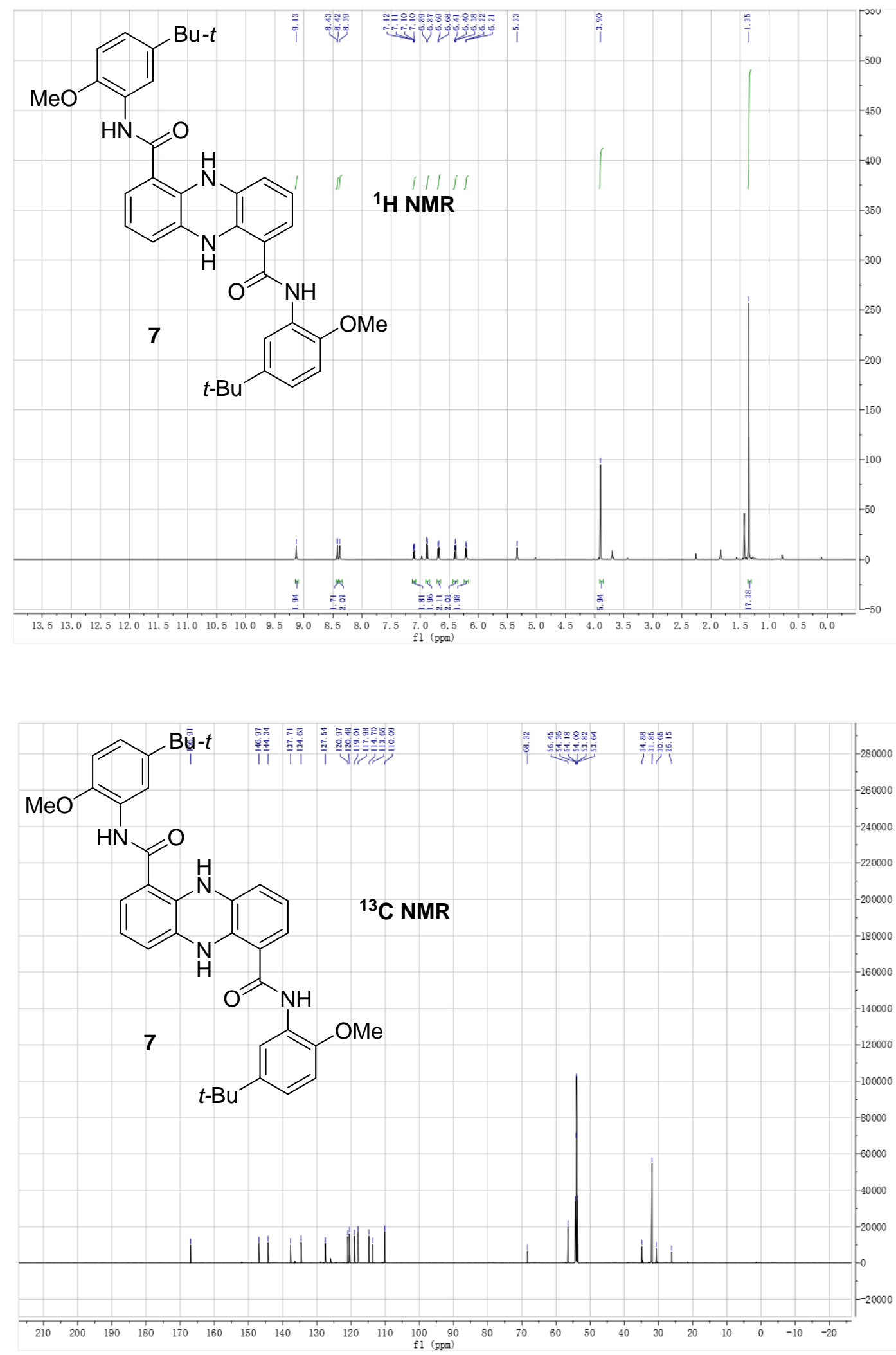

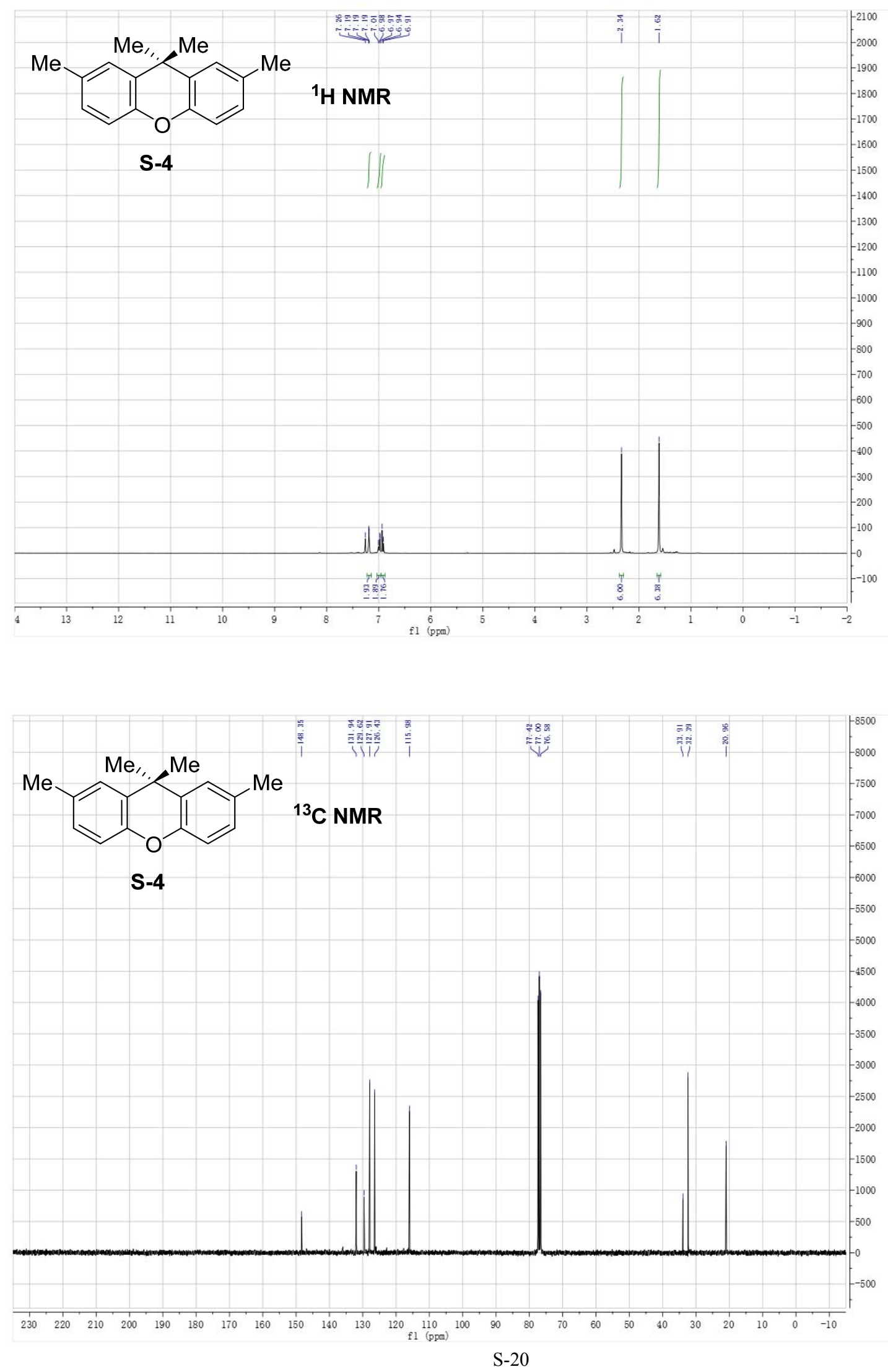

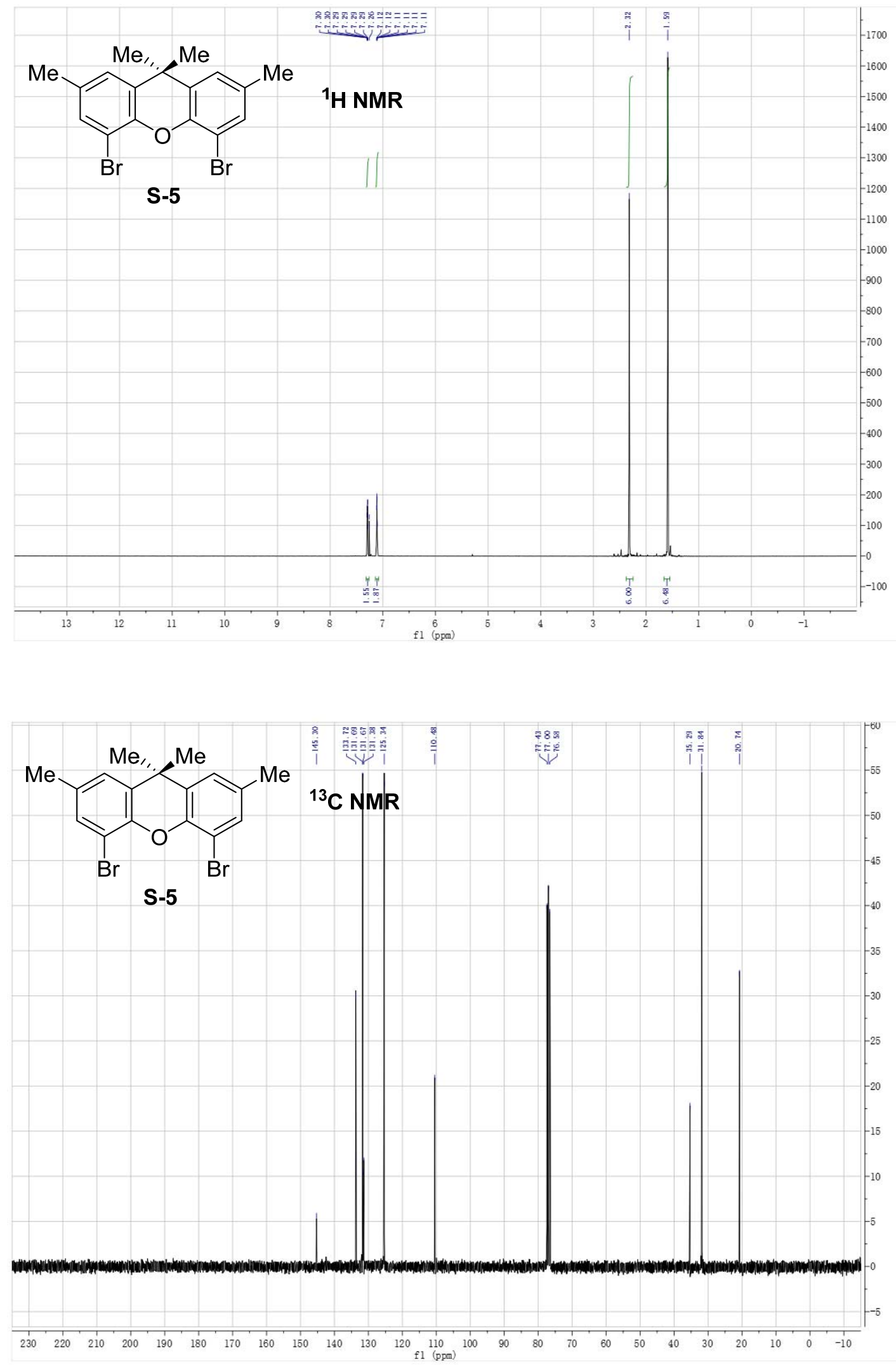

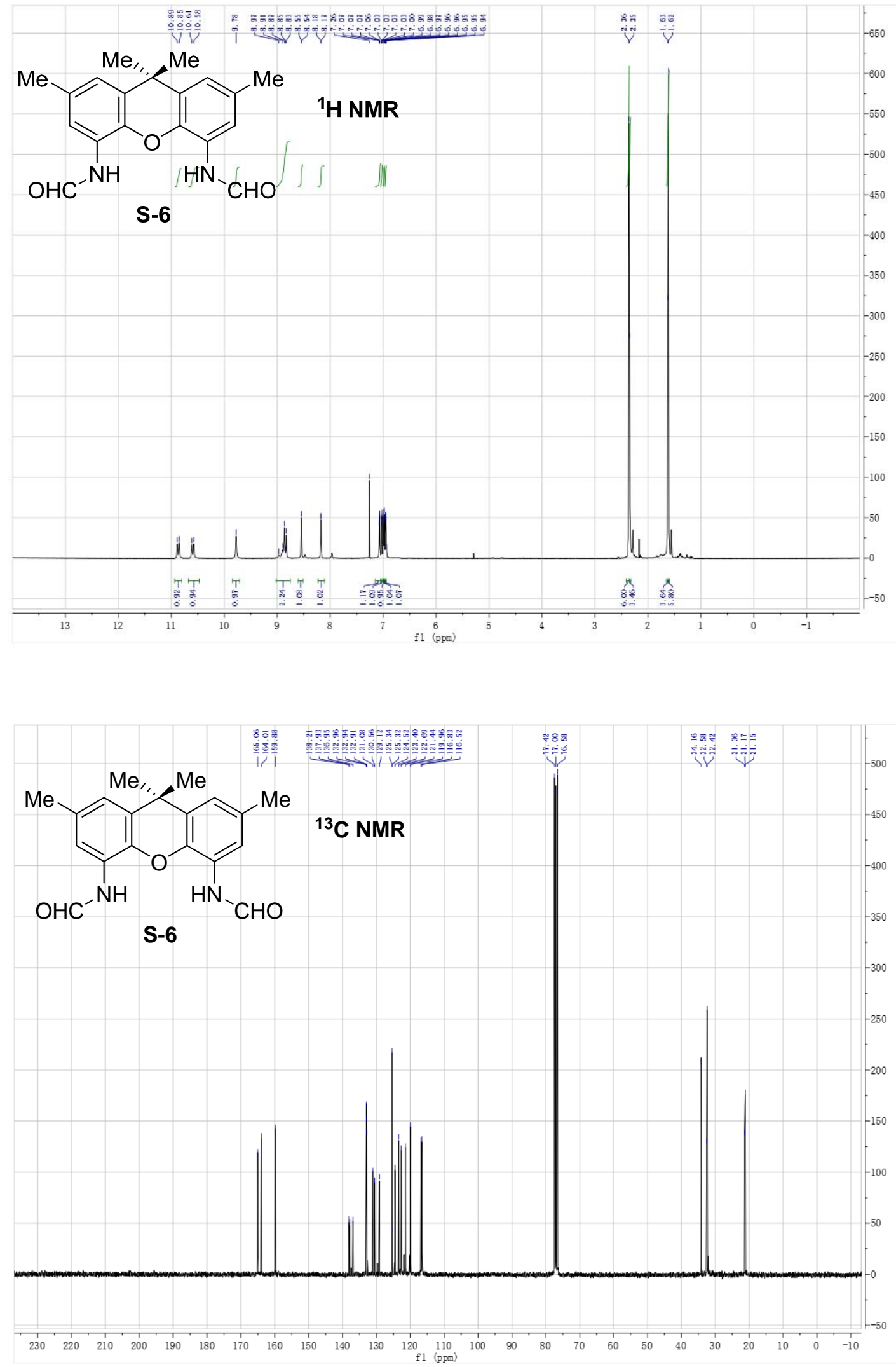

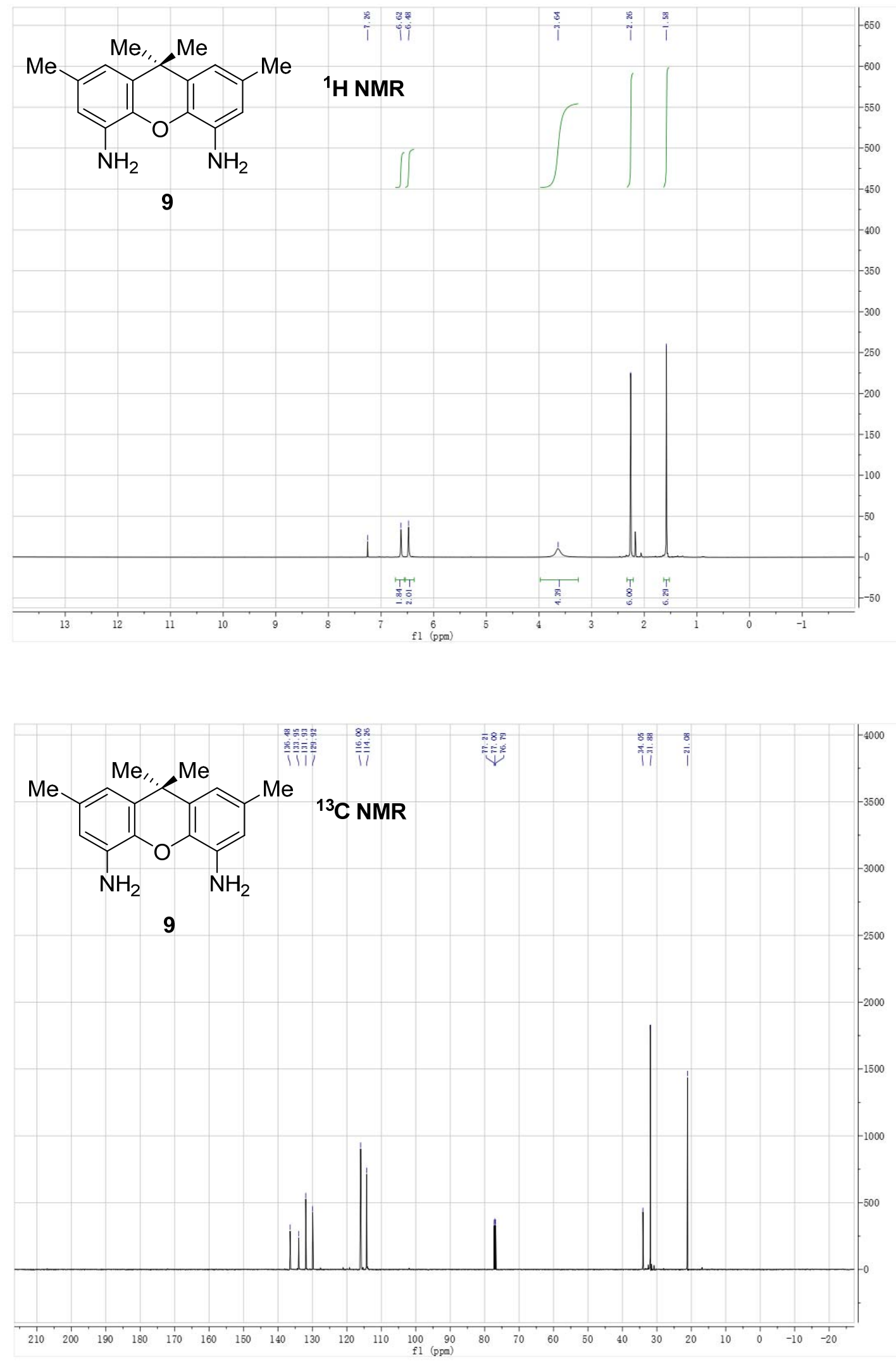

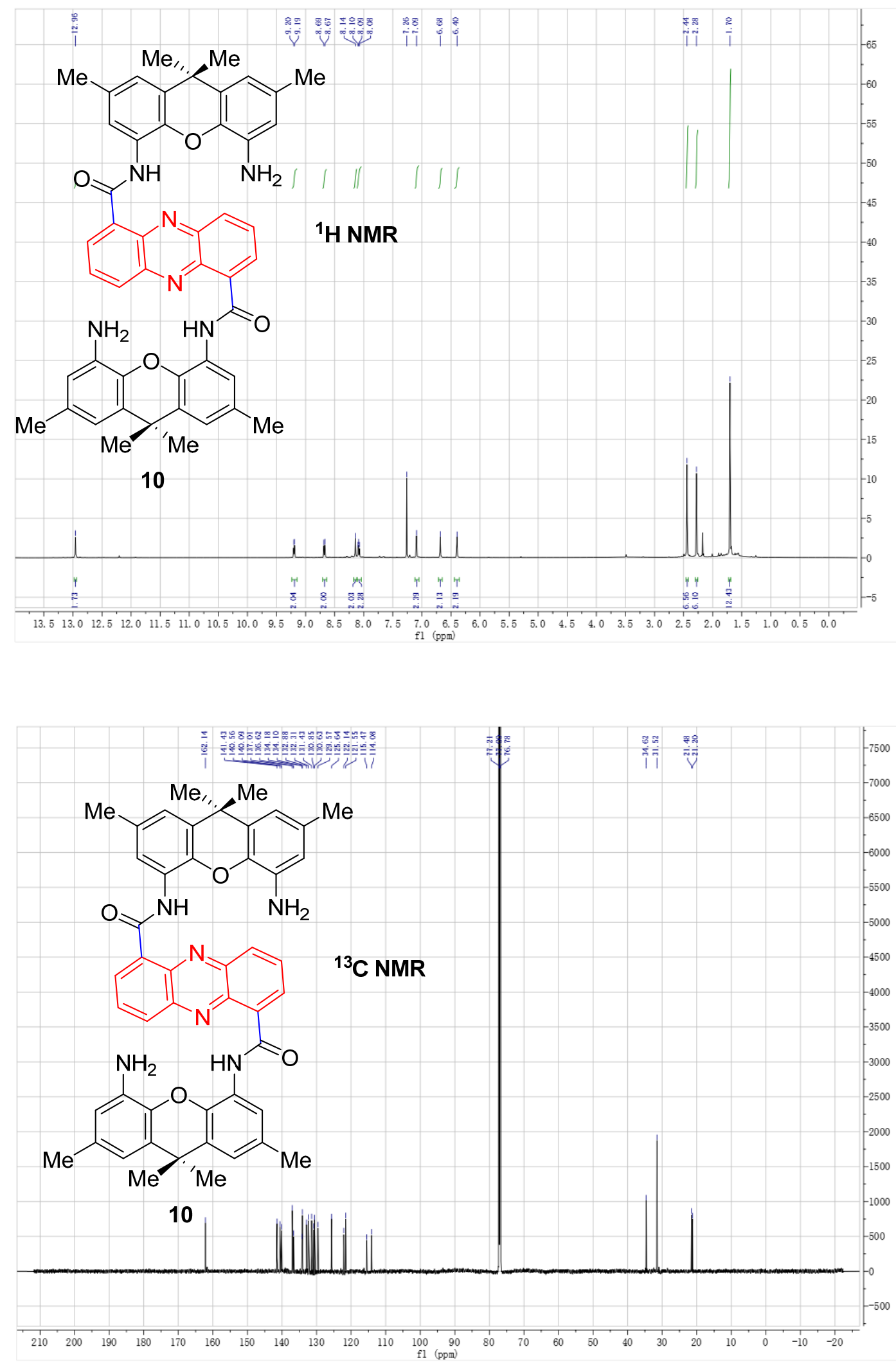

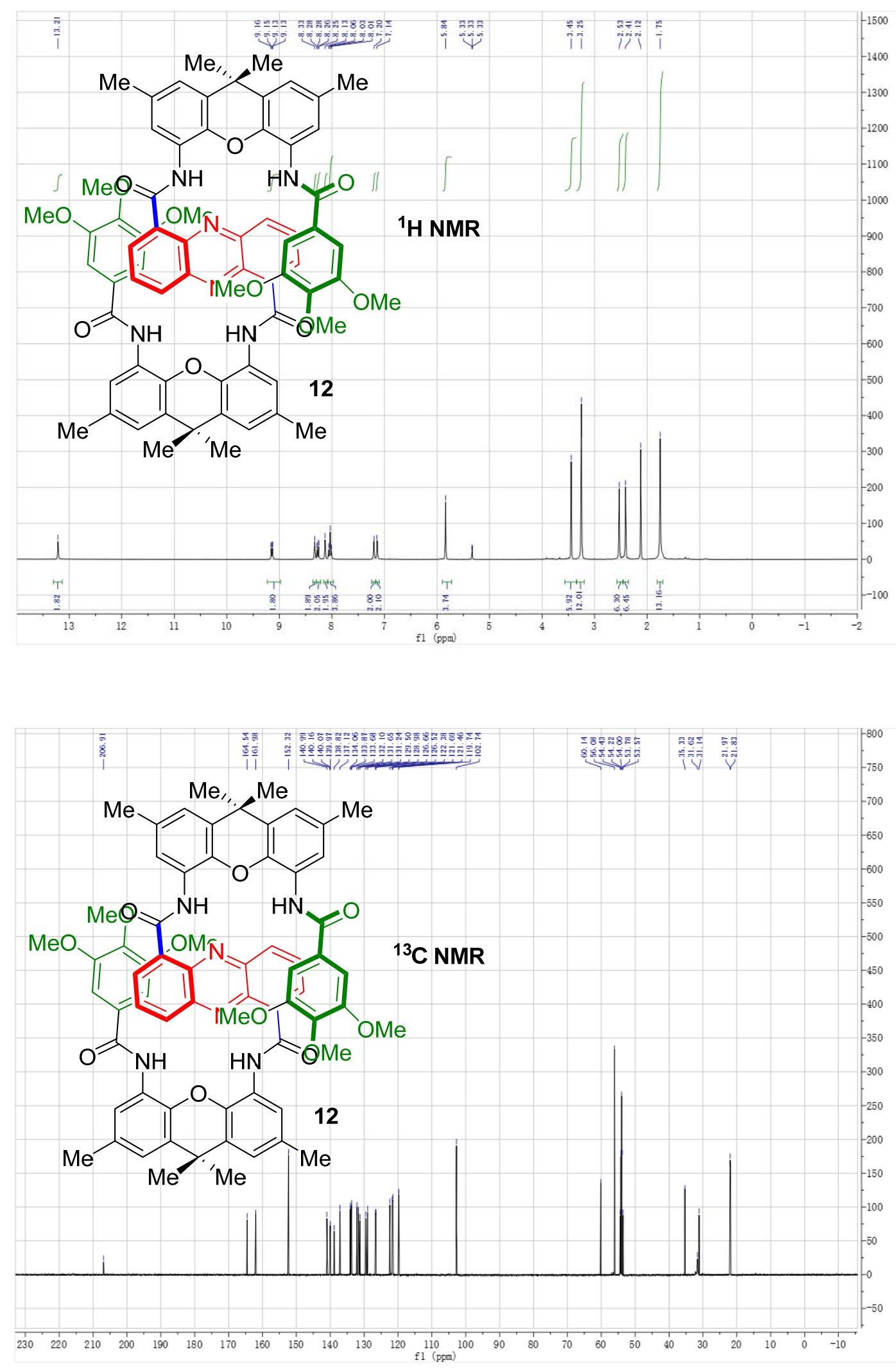

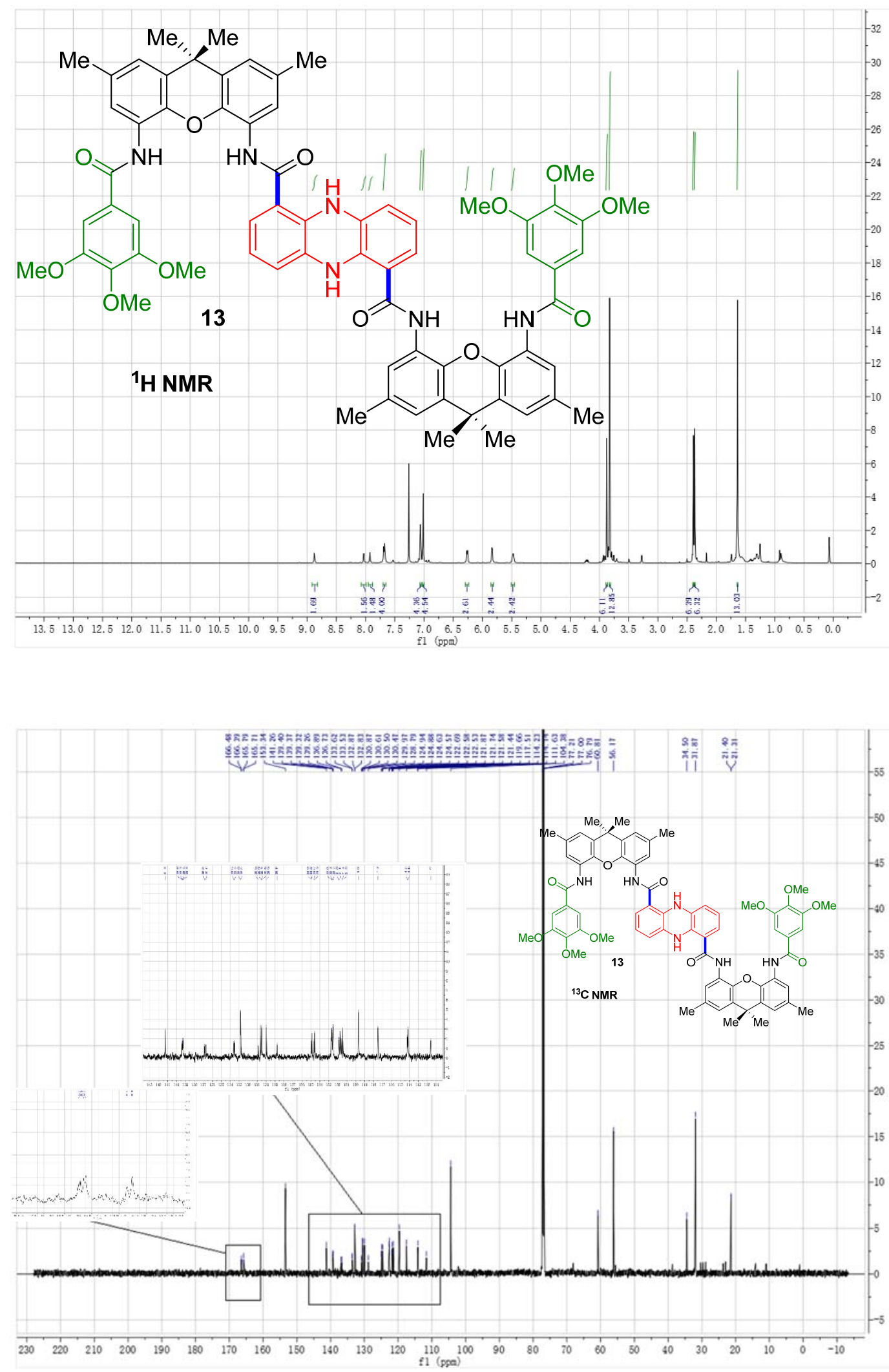

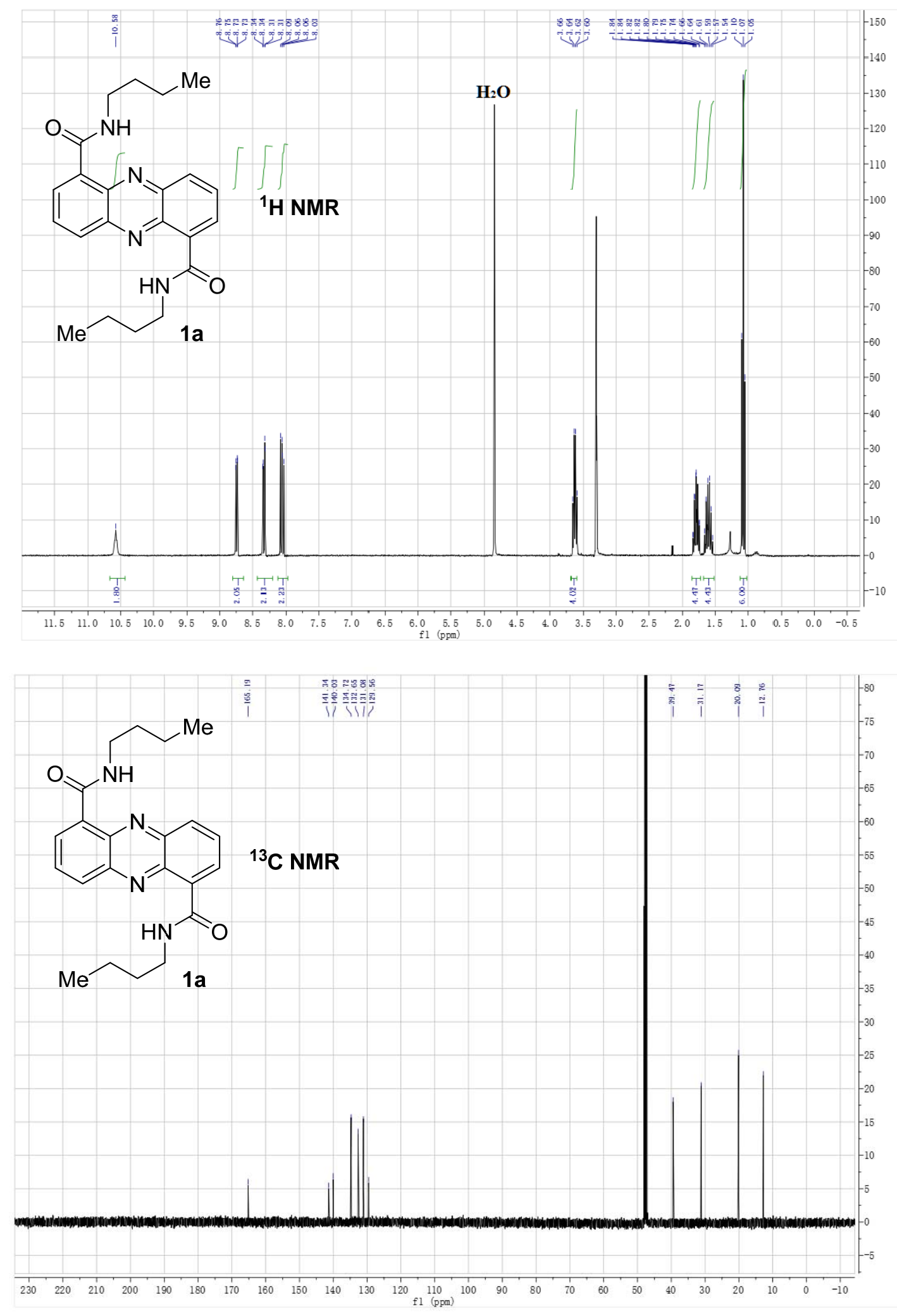


\section{2D NMR SPECTRA.}

NOESY Spectrum of $\mathbf{6}$ :

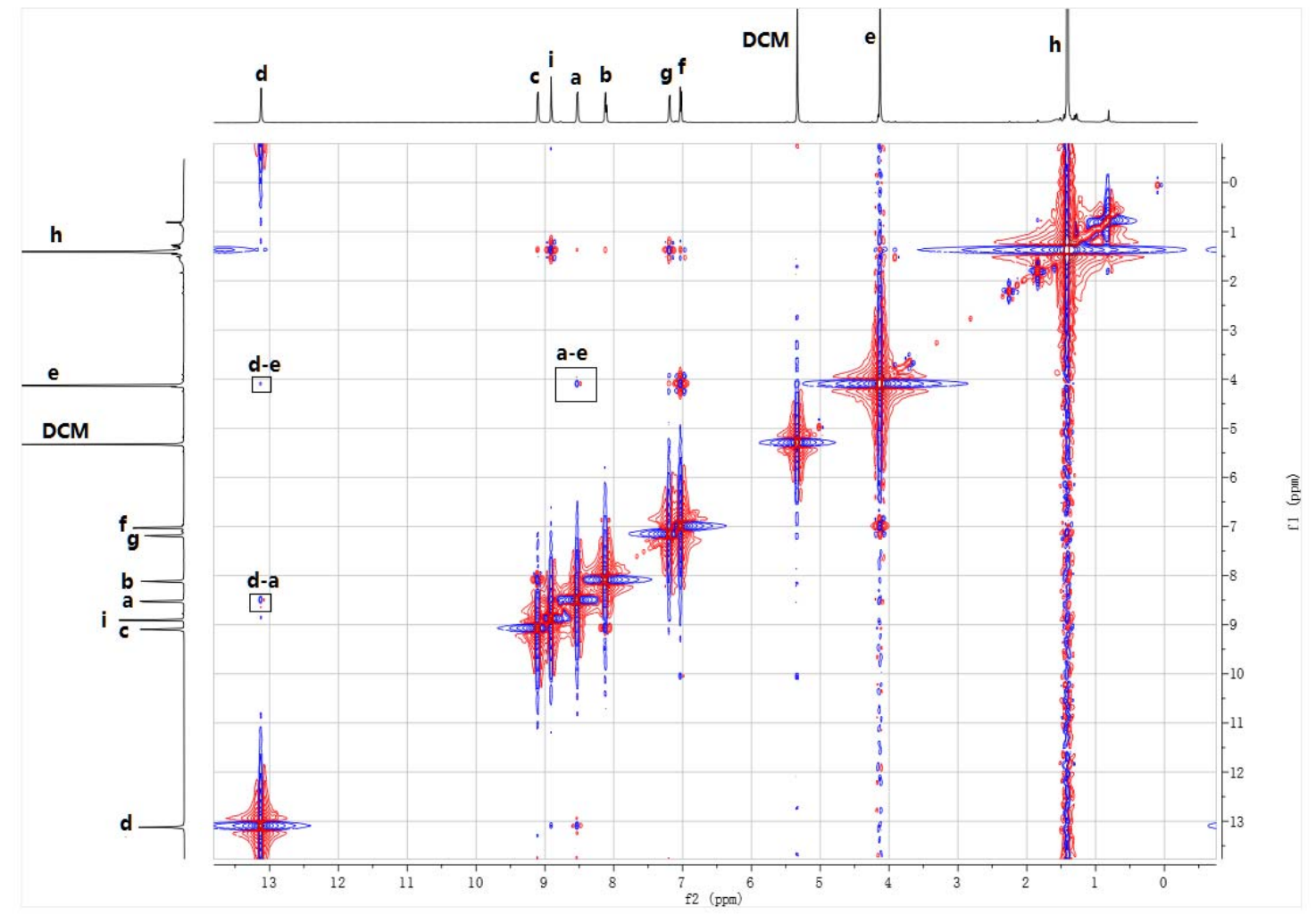

NOESY Spectrum of 7

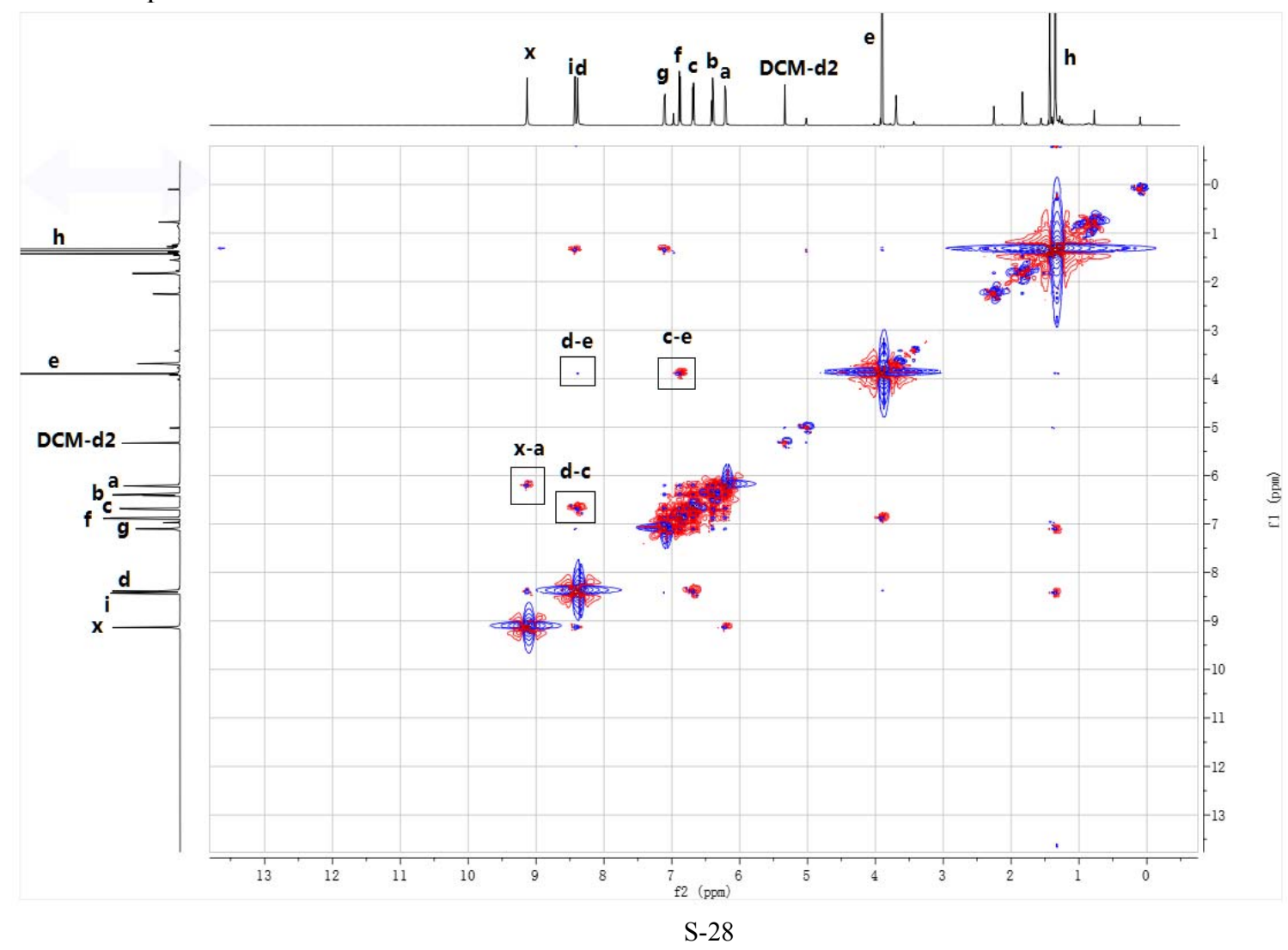


NOESY Spectrum of $\mathbf{1 2}$

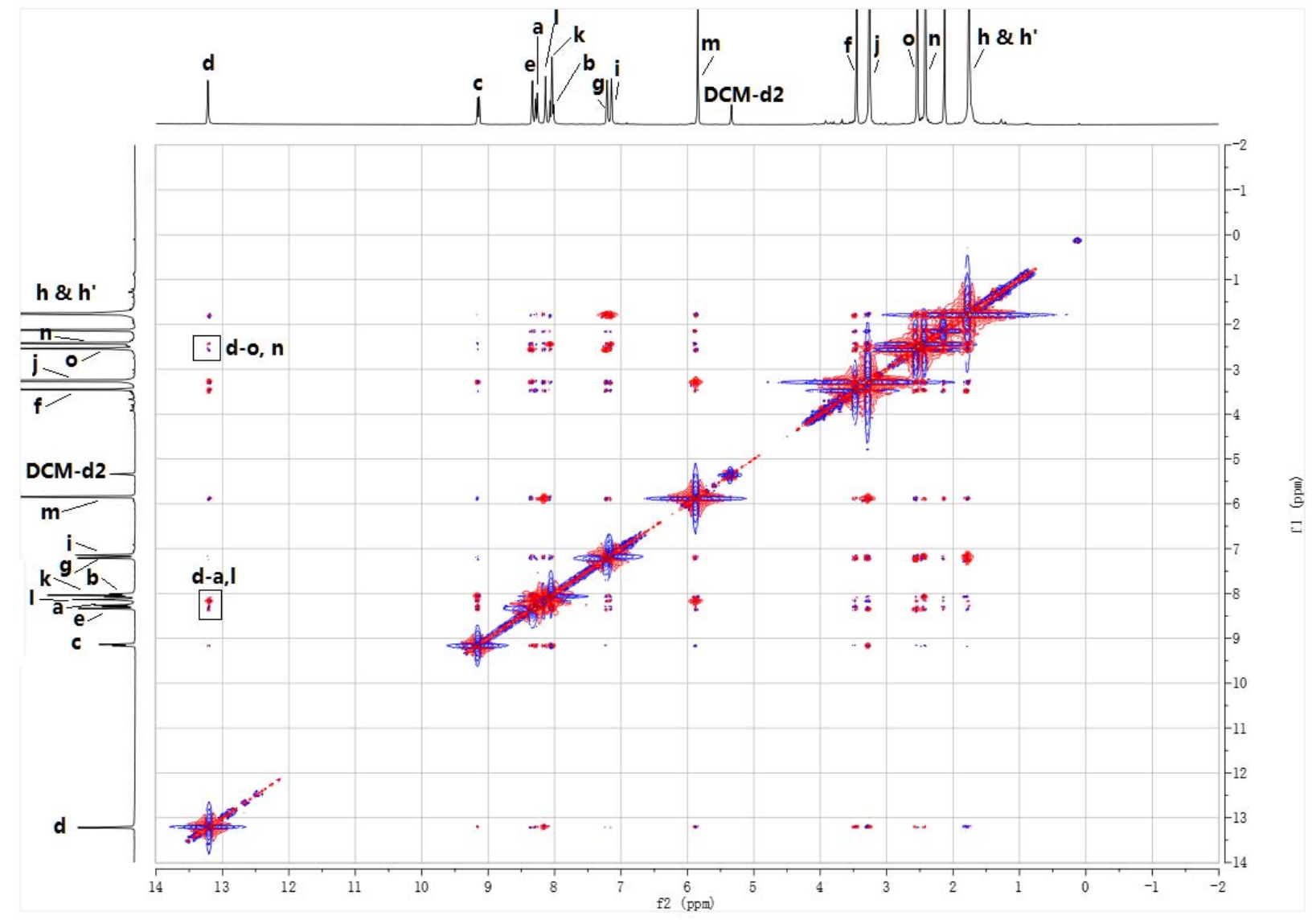

HMBC Spectrum of $\mathbf{1 2}$

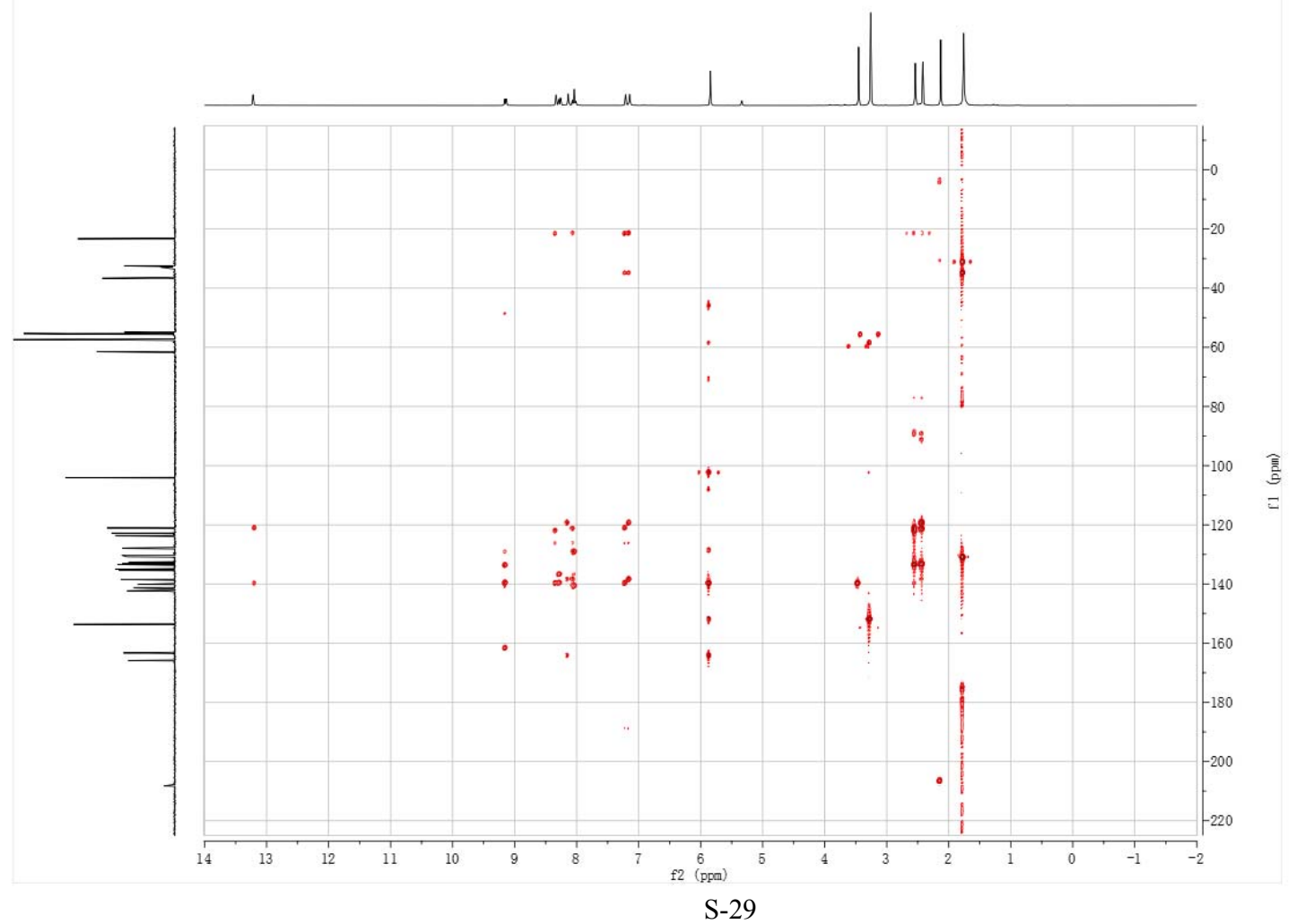


NOESY Spectrum of $\mathbf{1 3}$

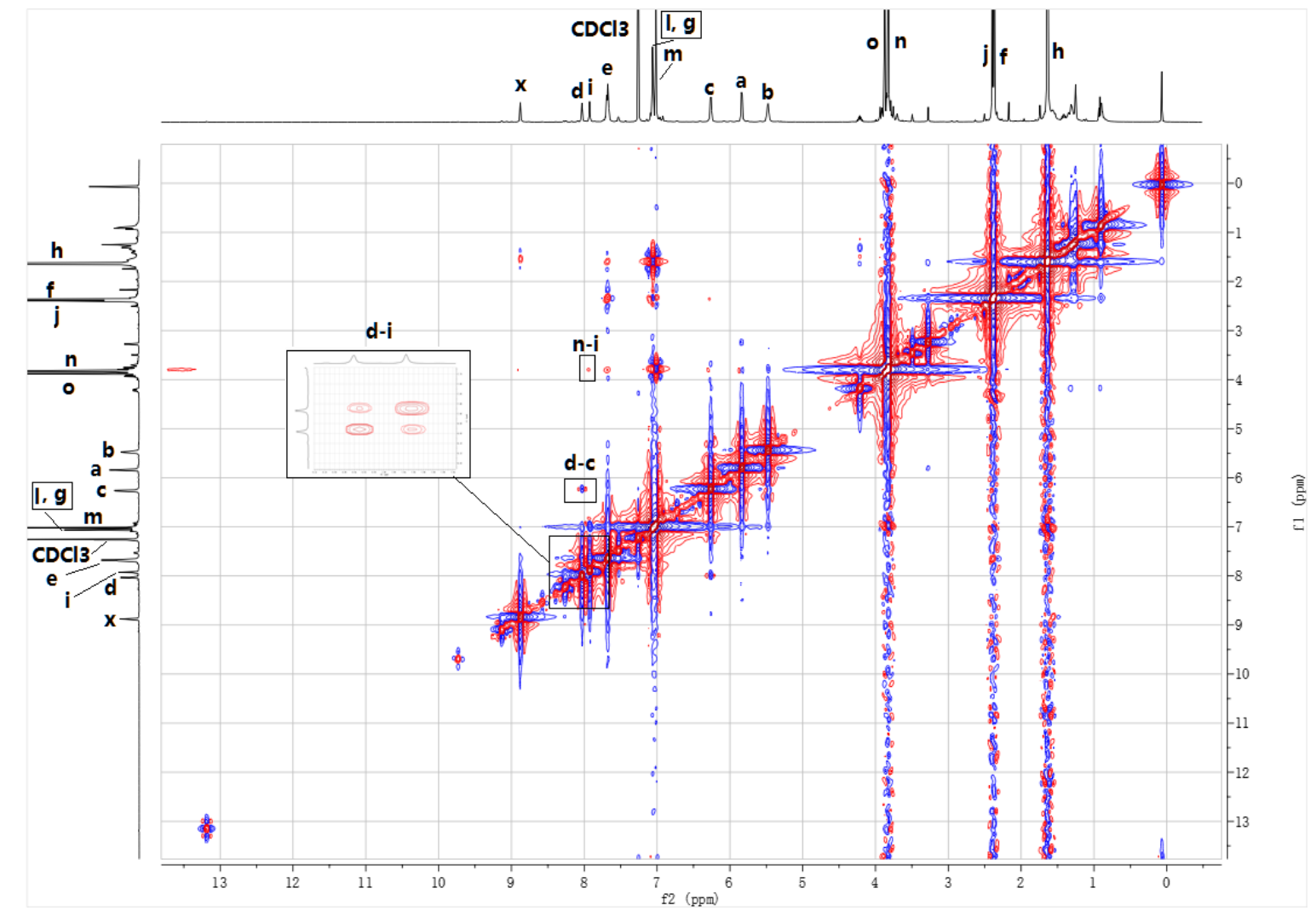

HSQC Spectrum of $\mathbf{1 3}$

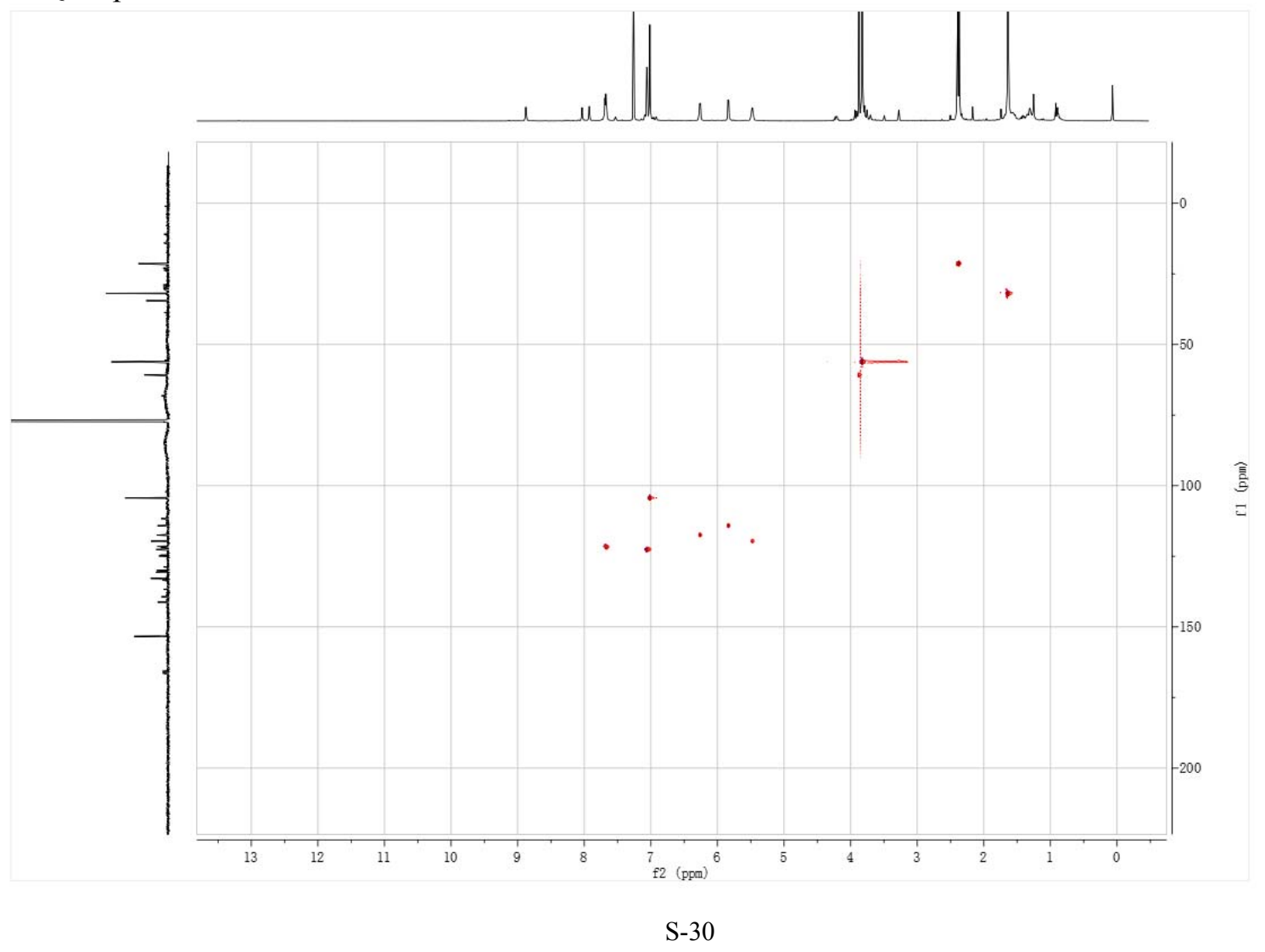


HMBC Spectrum of $\mathbf{1 3}$

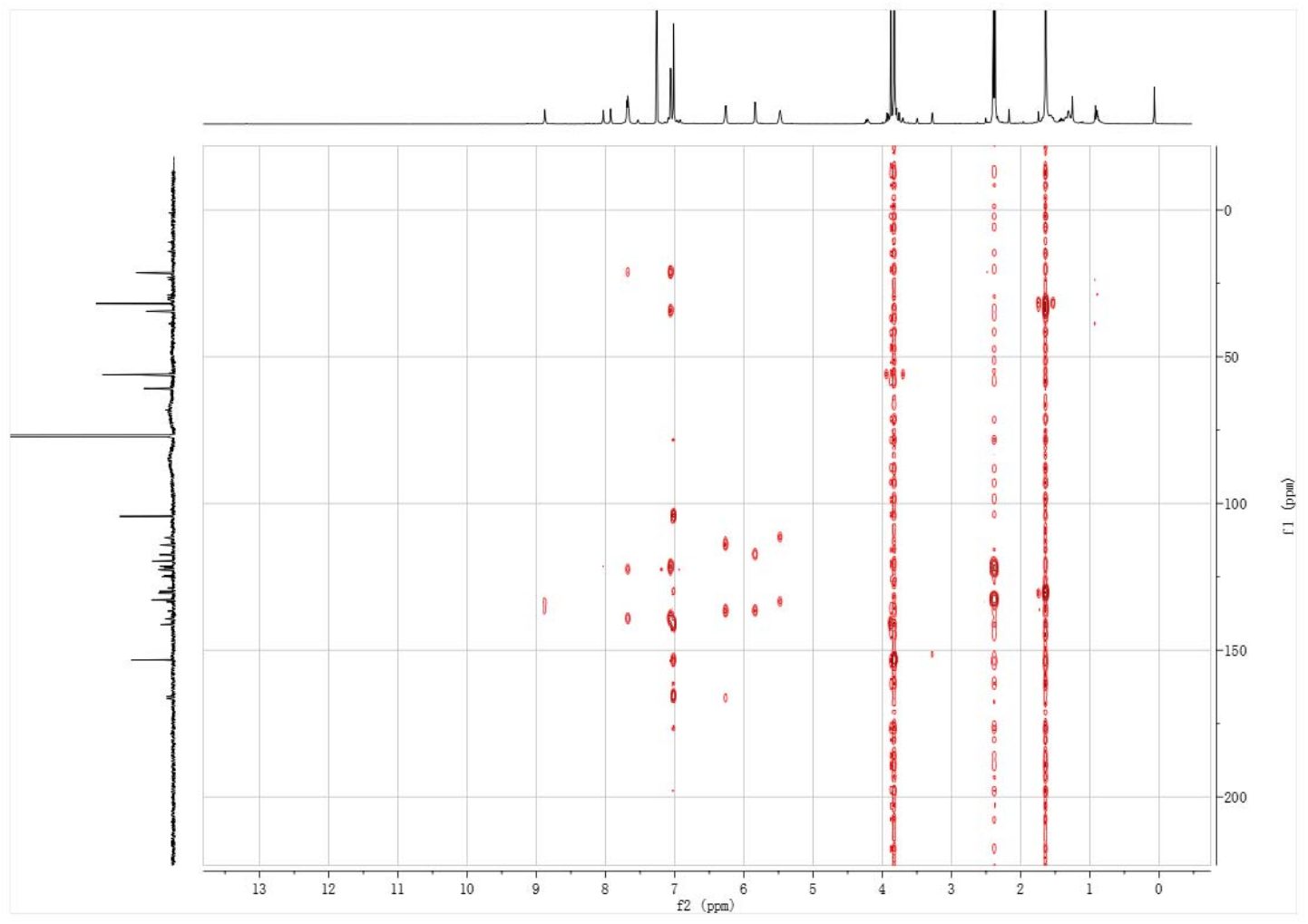




\section{X-RAY CRYSTALlOgRAPHIC DATA FOR 12.}

Table S-1. Crystal data and structure refinement for vb7519.

Identification code

Empirical formula

Formula weight

Temperature

Wavelength

Crystal system

Space group

Unit cell dimensions

Volume

Z

Density (calculated)

Absorption coefficient

$\mathrm{F}(000)$

Crystal size

Theta range for data collection

Index ranges

Reflections collected

Independent reflections

Completeness to theta $=67.679^{\circ}$

Absorption correction

Max. and min. transmission

Refinement method

Data / restraints / parameters

Goodness-of-fit on $\mathrm{F}^{2}$

Final $\mathrm{R}$ indices $[\mathrm{I}>2 \operatorname{sigma}(\mathrm{I})]$

$\mathrm{R}$ indices (all data)

Largest diff. peak and hole
v7519/1t/Venture/Yin-Birman

$\mathrm{C}_{72} \mathrm{H}_{68} \mathrm{Cl}_{12} \mathrm{~N}_{6} \mathrm{O}_{12}$

1634.72

$100(2) \mathrm{K}$

$1.54178 \AA$

Triclinic

P-1

$$
\begin{array}{ll}
\mathrm{a}=9.3674(5) \AA & =102.300(3)^{\circ} . \\
\mathrm{b}=14.1728(7) \AA & =103.122(3)^{\circ} . \\
\mathrm{c}=15.4346(9) \AA & =107.338(3)^{\circ} .
\end{array}
$$

1816.04(17) $\AA^{3}$

1

$1.495 \mathrm{Mg} / \mathrm{m}^{3}$

$4.740 \mathrm{~mm}^{-1}$

842

$0.182 \times 0.124 \times 0.093 \mathrm{~mm}^{3}$

3.084 to $68.314^{\circ}$.

$-11 \leq \mathrm{h} \leq 11,-17 \leq \mathrm{k} \leq 17,-18 \leq \mathrm{l} \leq 18$

18610

$6510[\mathrm{R}(\mathrm{int})=0.0454]$

$97.9 \%$

Semi-empirical from equivalents

0.5210 and 0.3976

Full-matrix least-squares on $\mathrm{F}^{2}$

$6510 / 1 / 470$

1.085

$\mathrm{R} 1=0.0904, \mathrm{wR} 2=0.2172$

$\mathrm{R} 1=0.1103, \mathrm{wR} 2=0.2348$

0.788 and -0.494 e. $\AA^{-3}$ 
Table S-2. Atomic coordinates ( x 104) and equivalent isotropic displacement parameters $\left(\AA^{2} \mathrm{x}\right.$ $10^{3}$ ) for vb7519. $\mathrm{U}(\mathrm{eq})$ is defined as one third of the trace of the orthogonalized $\mathrm{U}^{\mathrm{ij}}$ tensor.

\begin{tabular}{|c|c|c|c|c|}
\hline & $\mathrm{x}$ & $\mathrm{y}$ & $\mathrm{Z}$ & $\mathrm{U}(\mathrm{eq})$ \\
\hline $\mathrm{O}(1)$ & $1202(5)$ & $3644(3)$ & $5879(3)$ & $37(1)$ \\
\hline $\mathrm{O}(2)$ & $2619(5)$ & $5308(3)$ & $7378(3)$ & $32(1)$ \\
\hline $\mathrm{O}(3)$ & $5602(5)$ & $5823(3)$ & $8394(3)$ & $35(1)$ \\
\hline $\mathrm{O}(4)$ & $7090(5)$ & 2761(3) & $7113(3)$ & $42(1)$ \\
\hline $\mathrm{O}(5)$ & $5370(4)$ & $1686(3)$ & $3792(3)$ & $29(1)$ \\
\hline $\mathrm{O}(6)$ & $936(5)$ & 2336(3) & 2074(3) & $37(1)$ \\
\hline $\mathrm{N}(1)$ & $5569(5)$ & $2116(3)$ & $5593(3)$ & $28(1)$ \\
\hline $\mathrm{N}(2)$ & $3198(5)$ & $2323(3)$ & 2993(3) & $26(1)$ \\
\hline $\mathrm{N}(3)$ & $5075(5)$ & 5954(3) & $5530(3)$ & $28(1)$ \\
\hline $\mathrm{C}(1)$ & 2719(6) & $3817(4)$ & 6352(4) & $30(1)$ \\
\hline$C(2)$ & $3463(7)$ & 4713(4) & $7119(4)$ & $29(1)$ \\
\hline$C(3)$ & $5015(7)$ & 4951(4) & $7671(4)$ & $29(1)$ \\
\hline $\mathrm{C}(4)$ & $5808(7)$ & $4290(4)$ & $7441(4)$ & $29(1)$ \\
\hline$C(5)$ & $5053(7)$ & $3401(4)$ & $6677(4)$ & $28(1)$ \\
\hline$C(6)$ & $3516(6)$ & $3161(4)$ & $6130(4)$ & $28(1)$ \\
\hline$C(7)$ & $381(7)$ & $2722(5)$ & $5138(5)$ & $41(2)$ \\
\hline $\mathrm{C}(8)$ & 3004(8) & $6255(5)$ & 7138(4) & $37(1)$ \\
\hline$C(9)$ & $7153(8)$ & $6088(5)$ & $9019(4)$ & $40(1)$ \\
\hline$C(10)$ & 5996(7) & $2738(4)$ & $6493(4)$ & $31(1)$ \\
\hline $\mathrm{C}(11)$ & $6258(6)$ & $1425(4)$ & $5251(4)$ & $25(1)$ \\
\hline$C(12)$ & $7046(6)$ & 963(4) & 5803(4) & $28(1)$ \\
\hline$C(13)$ & $7654(6)$ & $252(4)$ & $5403(4)$ & $29(1)$ \\
\hline$C(14)$ & $7447(6)$ & $-2(4)$ & $4455(4)$ & $30(1)$ \\
\hline$C(15)$ & $6667(6)$ & $446(4)$ & $3879(4)$ & $27(1)$ \\
\hline$C(16)$ & 6496(7) & 247(4) & 2843(4) & $30(1)$ \\
\hline$C(17)$ & $5021(7)$ & $409(4)$ & $2350(4)$ & $29(1)$ \\
\hline$C(18)$ & $4148(7)$ & $-73(4)$ & $1417(4)$ & $32(1)$ \\
\hline$C(19)$ & 2904(7) & 179(4) & 978(4) & $32(1)$ \\
\hline$C(20)$ & 2531(7) & $971(4)$ & 1499(4) & $32(1)$ \\
\hline$C(21)$ & $3370(7)$ & $1458(4)$ & $2422(4)$ & $28(1)$ \\
\hline$C(22)$ & $4589(6)$ & 1164(4) & $2853(4)$ & $27(1)$ \\
\hline$C(23)$ & $6114(6)$ & $1175(4)$ & $4300(4)$ & $27(1)$ \\
\hline$C(24)$ & $8489(7)$ & $-241(5)$ & 6011(4) & $33(1)$ \\
\hline$C(25)$ & $7945(7)$ & $1060(5)$ & $2738(4)$ & $35(1)$ \\
\hline$C(26)$ & 6451(8) & $-846(5)$ & $2393(4)$ & $39(1)$ \\
\hline$C(27)$ & 2002(8) & $-343(5)$ & $-44(4)$ & $40(1)$ \\
\hline $\mathrm{C}(28)$ & 2078(7) & 2731(4) & 2772(4) & $28(1)$ \\
\hline$C(29)$ & $2344(6)$ & $3756(4)$ & $3464(4)$ & $27(1)$ \\
\hline$C(30)$ & $1153(7)$ & $4123(4)$ & $3277(4)$ & $31(1)$ \\
\hline $\mathrm{C}(31)$ & $1204(7)$ & $5078(4)$ & $3835(4)$ & $31(1)$ \\
\hline $\mathrm{C}(32)$ & 2499(6) & $5666(4)$ & 4584(4) & $27(1)$ \\
\hline$C(33)$ & $3799(6)$ & $5341(4)$ & $4806(4)$ & $26(1)$ \\
\hline$C(34)$ & 6271(6) & $5635(4)$ & $5746(4)$ & $26(1)$ \\
\hline $\mathrm{C}(1 \mathrm{~S})$ & 6571(7) & $3240(5)$ & $455(4)$ & $36(1)$ \\
\hline $\mathrm{C}(2 \mathrm{~S})$ & $9765(8)$ & $7018(5)$ & $1765(5)$ & $43(2)$ \\
\hline $\mathrm{Cl}(1)$ & $8406(2)$ & $3094(1)$ & $817(1)$ & $44(1)$ \\
\hline $\mathrm{Cl}(2)$ & $5062(2)$ & $2135(1)$ & $456(1)$ & $43(1)$ \\
\hline $\mathrm{Cl}(3)$ & $6240(2)$ & $3379(1)$ & $-676(1)$ & $50(1)$ \\
\hline $\mathrm{Cl}(4)$ & $10785(2)$ & $8055(1)$ & $1438(1)$ & $50(1)$ \\
\hline $\mathrm{Cl}(5)$ & $9650(2)$ & $5824(1)$ & $1072(1)$ & $52(1)$ \\
\hline $\mathrm{Cl}(6)$ & $7835(2)$ & $6998(2)$ & $1689(2)$ & $54(1)$ \\
\hline $\mathrm{Cl}\left(6^{\prime}\right)$ & $8460(30)$ & $7270(20)$ & $2370(20)$ & $54(1)$ \\
\hline
\end{tabular}


Table S-3. Bond lengths $[\AA]$ and angles $\left[{ }^{\circ}\right]$ for vb7519.

\begin{tabular}{|c|c|c|c|c|c|}
\hline $\mathrm{O}(1)-\mathrm{C}(1)$ & $1.363(7)$ & $\mathrm{C}(19)-\mathrm{C}(27)$ & $1.507(8)$ & $\mathrm{C}(4)-\mathrm{C}(3)-\mathrm{C}(2)$ & $119.3(5)$ \\
\hline $\mathrm{O}(1)-\mathrm{C}(7)$ & $1.404(7)$ & $C(20)-C(21)$ & $1.369(8)$ & $\mathrm{C}(5)-\mathrm{C}(4)-\mathrm{C}(3)$ & $119.8(5)$ \\
\hline $\mathrm{O}(2)-\mathrm{C}(2)$ & $1.376(6)$ & $\mathrm{C}(20)-\mathrm{H}(20)$ & 0.9500 & $\mathrm{C}(5)-\mathrm{C}(4)-\mathrm{H}(4)$ & 120.1 \\
\hline $\mathrm{O}(2)-\mathrm{C}(8)$ & $1.434(7)$ & $C(21)-C(22)$ & $1.406(8)$ & $\mathrm{C}(3)-\mathrm{C}(4)-\mathrm{H}(4)$ & 120.1 \\
\hline $\mathrm{O}(3)-\mathrm{C}(3)$ & $1.343(7)$ & $\mathrm{C}(24)-\mathrm{H}(24 \mathrm{~A})$ & 0.9800 & $C(4)-C(5)-C(6)$ & $121.1(5)$ \\
\hline $\mathrm{O}(3)-\mathrm{C}(9)$ & $1.438(7)$ & $\mathrm{C}(24)-\mathrm{H}(24 \mathrm{~B})$ & 0.9800 & $C(4)-C(5)-C(10)$ & $115.8(5)$ \\
\hline $\mathrm{O}(4)-\mathrm{C}(10)$ & $1.221(7)$ & $\mathrm{C}(24)-\mathrm{H}(24 \mathrm{C})$ & 0.9800 & $C(6)-C(5)-C(10)$ & $123.1(5)$ \\
\hline $\mathrm{O}(5)-\mathrm{C}(23)$ & $1.385(6)$ & $\mathrm{C}(25)-\mathrm{H}(25 \mathrm{~A})$ & 0.9800 & $C(1)-C(6)-C(5)$ & $119.5(5)$ \\
\hline $\mathrm{O}(5)-\mathrm{C}(22)$ & $1.387(6)$ & $\mathrm{C}(25)-\mathrm{H}(25 \mathrm{~B})$ & 0.9800 & $\mathrm{C}(1)-\mathrm{C}(6)-\mathrm{H}(6)$ & 120.2 \\
\hline $\mathrm{O}(6)-\mathrm{C}(28)$ & $1.217(7)$ & $\mathrm{C}(25)-\mathrm{H}(25 \mathrm{C})$ & 0.9800 & $\mathrm{C}(5)-\mathrm{C}(6)-\mathrm{H}(6)$ & 120.2 \\
\hline $\mathrm{N}(1)-\mathrm{C}(10)$ & $1.371(7)$ & $\mathrm{C}(26)-\mathrm{H}(26 \mathrm{~A})$ & 0.9800 & $\mathrm{O}(1)-\mathrm{C}(7)-\mathrm{H}(7 \mathrm{~A})$ & 109.5 \\
\hline $\mathrm{N}(1)-\mathrm{C}(11)$ & $1.403(7)$ & $\mathrm{C}(26)-\mathrm{H}(26 \mathrm{~B})$ & 0.9800 & $\mathrm{O}(1)-\mathrm{C}(7)-\mathrm{H}(7 \mathrm{~B})$ & 109.5 \\
\hline $\mathrm{N}(1)-\mathrm{H}(1)$ & 0.8800 & $\mathrm{C}(26)-\mathrm{H}(26 \mathrm{C})$ & 0.9800 & $\mathrm{H}(7 \mathrm{~A})-\mathrm{C}(7)-\mathrm{H}(7 \mathrm{~B})$ & 109.5 \\
\hline $\mathrm{N}(2)-\mathrm{C}(28)$ & $1.354(7)$ & $\mathrm{C}(27)-\mathrm{H}(27 \mathrm{~A})$ & 0.9800 & $\mathrm{O}(1)-\mathrm{C}(7)-\mathrm{H}(7 \mathrm{C})$ & 109.5 \\
\hline $\mathrm{N}(2)-\mathrm{C}(21)$ & $1.423(6)$ & $\mathrm{C}(27)-\mathrm{H}(27 \mathrm{~B})$ & 0.9800 & $\mathrm{H}(7 \mathrm{~A})-\mathrm{C}(7)-\mathrm{H}(7 \mathrm{C})$ & 109.5 \\
\hline $\mathrm{N}(2)-\mathrm{H}(2)$ & 0.8800 & $\mathrm{C}(27)-\mathrm{H}(27 \mathrm{C})$ & 0.9800 & $\mathrm{H}(7 \mathrm{~B})-\mathrm{C}(7)-\mathrm{H}(7 \mathrm{C})$ & 109.5 \\
\hline $\mathrm{N}(3)-\mathrm{C}(34)$ & $1.330(7)$ & $C(28)-C(29)$ & $1.523(7)$ & $\mathrm{O}(2)-\mathrm{C}(8)-\mathrm{H}(8 \mathrm{~A})$ & 109.5 \\
\hline $\mathrm{N}(3)-\mathrm{C}(33)$ & $1.339(7)$ & $C(29)-C(30)$ & $1.364(8)$ & $\mathrm{O}(2)-\mathrm{C}(8)-\mathrm{H}(8 \mathrm{~B})$ & 109.5 \\
\hline$C(1)-C(6)$ & $1.390(8)$ & $\mathrm{C}(29)-\mathrm{C}(34) \# 1$ & $1.442(8)$ & $\mathrm{H}(8 \mathrm{~A})-\mathrm{C}(8)-\mathrm{H}(8 \mathrm{~B})$ & 109.5 \\
\hline$C(1)-C(2)$ & $1.399(8)$ & $C(30)-C(31)$ & $1.422(8)$ & $\mathrm{O}(2)-\mathrm{C}(8)-\mathrm{H}(8 \mathrm{C})$ & 109.5 \\
\hline$C(2)-C(3)$ & $1.407(8)$ & $\mathrm{C}(30)-\mathrm{H}(30)$ & 0.9500 & $\mathrm{H}(8 \mathrm{~A})-\mathrm{C}(8)-\mathrm{H}(8 \mathrm{C})$ & 109.5 \\
\hline$C(3)-C(4)$ & $1.396(8)$ & $C(31)-C(32)$ & $1.361(8)$ & $\mathrm{H}(8 \mathrm{~B})-\mathrm{C}(8)-\mathrm{H}(8 \mathrm{C})$ & 109.5 \\
\hline$C(4)-C(5)$ & $1.390(8)$ & $\mathrm{C}(31)-\mathrm{H}(31)$ & 0.9500 & $\mathrm{O}(3)-\mathrm{C}(9)-\mathrm{H}(9 \mathrm{~A})$ & 109.5 \\
\hline $\mathrm{C}(4)-\mathrm{H}(4)$ & 0.9500 & $C(32)-C(33)$ & $1.423(7)$ & $\mathrm{O}(3)-\mathrm{C}(9)-\mathrm{H}(9 \mathrm{~B})$ & 109.5 \\
\hline$C(5)-C(6)$ & $1.391(8)$ & $\mathrm{C}(32)-\mathrm{H}(32)$ & 0.9500 & $\mathrm{H}(9 \mathrm{~A})-\mathrm{C}(9)-\mathrm{H}(9 \mathrm{~B})$ & 109.5 \\
\hline$C(5)-C(10)$ & $1.497(7)$ & $\mathrm{C}(33)-\mathrm{C}(34) \# 1$ & $1.436(7)$ & $\mathrm{O}(3)-\mathrm{C}(9)-\mathrm{H}(9 \mathrm{C})$ & 109.5 \\
\hline $\mathrm{C}(6)-\mathrm{H}(6)$ & 0.9500 & $\mathrm{C}(1 \mathrm{~S})-\mathrm{Cl}(3)$ & $1.766(6)$ & $\mathrm{H}(9 \mathrm{~A})-\mathrm{C}(9)-\mathrm{H}(9 \mathrm{C})$ & 109.5 \\
\hline $\mathrm{C}(7)-\mathrm{H}(7 \mathrm{~A})$ & 0.9800 & $\mathrm{C}(1 \mathrm{~S})-\mathrm{Cl}(2)$ & $1.771(6)$ & $\mathrm{H}(9 \mathrm{~B})-\mathrm{C}(9)-\mathrm{H}(9 \mathrm{C})$ & 109.5 \\
\hline $\mathrm{C}(7)-\mathrm{H}(7 \mathrm{~B})$ & 0.9800 & $\mathrm{C}(1 \mathrm{~S})-\mathrm{Cl}(1)$ & $1.771(6)$ & $\mathrm{O}(4)-\mathrm{C}(10)-\mathrm{N}(1)$ & $122.4(5)$ \\
\hline $\mathrm{C}(7)-\mathrm{H}(7 \mathrm{C})$ & 0.9800 & $\mathrm{C}(1 \mathrm{~S})-\mathrm{H}(1 \mathrm{~S})$ & 1.0000 & $\mathrm{O}(4)-\mathrm{C}(10)-\mathrm{C}(5)$ & $121.3(5)$ \\
\hline $\mathrm{C}(8)-\mathrm{H}(8 \mathrm{~A})$ & 0.9800 & $\mathrm{C}(2 \mathrm{~S})-\mathrm{Cl}(4)$ & $1.740(7)$ & $\mathrm{N}(1)-\mathrm{C}(10)-\mathrm{C}(5)$ & $116.4(5)$ \\
\hline $\mathrm{C}(8)-\mathrm{H}(8 \mathrm{~B})$ & 0.9800 & $\mathrm{C}(2 \mathrm{~S})-\mathrm{Cl}(5)$ & $1.758(7)$ & $\mathrm{C}(12)-\mathrm{C}(11)-\mathrm{C}(23)$ & $118.7(5)$ \\
\hline $\mathrm{C}(8)-\mathrm{H}(8 \mathrm{C})$ & 0.9800 & $\mathrm{C}(2 \mathrm{~S})-\mathrm{Cl}\left(6^{\prime}\right)$ & $1.769(12)$ & $\mathrm{C}(12)-\mathrm{C}(11)-\mathrm{N}(1)$ & $123.4(5)$ \\
\hline $\mathrm{C}(9)-\mathrm{H}(9 \mathrm{~A})$ & 0.9800 & $\mathrm{C}(2 \mathrm{~S})-\mathrm{Cl}(6)$ & $1.775(7)$ & $\mathrm{C}(23)-\mathrm{C}(11)-\mathrm{N}(1)$ & $117.8(4)$ \\
\hline C(9)-H(9B) & 0.9800 & $\mathrm{C}(2 \mathrm{~S})-\mathrm{H}(2 \mathrm{~S})$ & 1.0000 & $C(11)-C(12)-C(13)$ & $120.1(5)$ \\
\hline C(9)-H(9C) & 0.9800 & $\mathrm{C}(1)-\mathrm{O}(1)-\mathrm{C}(7)$ & $117.4(4)$ & $\mathrm{C}(11)-\mathrm{C}(12)-\mathrm{H}(12)$ & 120.0 \\
\hline$C(11)-C(12)$ & $1.392(7)$ & $\mathrm{C}(2)-\mathrm{O}(2)-\mathrm{C}(8)$ & $113.2(4)$ & $\mathrm{C}(13)-\mathrm{C}(12)-\mathrm{H}(12)$ & 120.0 \\
\hline$C(11)-C(23)$ & $1.399(8)$ & $\mathrm{C}(3)-\mathrm{O}(3)-\mathrm{C}(9)$ & $118.2(4)$ & $\mathrm{C}(14)-\mathrm{C}(13)-\mathrm{C}(12)$ & $119.7(5)$ \\
\hline$C(12)-C(13)$ & $1.399(7)$ & $\mathrm{C}(23)-\mathrm{O}(5)-\mathrm{C}(22)$ & $116.5(4)$ & $C(14)-C(13)-C(24)$ & $121.2(5)$ \\
\hline $\mathrm{C}(12)-\mathrm{H}(12)$ & 0.9500 & $\mathrm{C}(10)-\mathrm{N}(1)-\mathrm{C}(11)$ & $127.0(5)$ & $\mathrm{C}(12)-\mathrm{C}(13)-\mathrm{C}(24)$ & $119.1(5)$ \\
\hline$C(13)-C(14)$ & $1.383(8)$ & $\mathrm{C}(10)-\mathrm{N}(1)-\mathrm{H}(1)$ & 116.5 & $C(13)-C(14)-C(15)$ & $121.9(5)$ \\
\hline$C(13)-C(24)$ & $1.501(7)$ & $\mathrm{C}(11)-\mathrm{N}(1)-\mathrm{H}(1)$ & 116.5 & $\mathrm{C}(13)-\mathrm{C}(14)-\mathrm{H}(14)$ & 119.0 \\
\hline$C(14)-C(15)$ & $1.396(8)$ & $\mathrm{C}(28)-\mathrm{N}(2)-\mathrm{C}(21)$ & $127.2(5)$ & $\mathrm{C}(15)-\mathrm{C}(14)-\mathrm{H}(14)$ & 119.0 \\
\hline $\mathrm{C}(14)-\mathrm{H}(14)$ & 0.9500 & $\mathrm{C}(28)-\mathrm{N}(2)-\mathrm{H}(2)$ & 116.4 & $C(23)-C(15)-C(14)$ & $117.2(5)$ \\
\hline$C(15)-C(23)$ & $1.395(7)$ & $\mathrm{C}(21)-\mathrm{N}(2)-\mathrm{H}(2)$ & 116.4 & $C(23)-C(15)-C(16)$ & $118.5(5)$ \\
\hline$C(15)-C(16)$ & $1.526(8)$ & $\mathrm{C}(34)-\mathrm{N}(3)-\mathrm{C}(33)$ & $118.9(5)$ & $C(14)-C(15)-C(16)$ & $124.1(5)$ \\
\hline$C(16)-C(17)$ & $1.525(8)$ & $\mathrm{O}(1)-\mathrm{C}(1)-\mathrm{C}(6)$ & $124.4(5)$ & $C(17)-C(16)-C(15)$ & $109.3(4)$ \\
\hline$C(16)-C(26)$ & $1.541(7)$ & $\mathrm{O}(1)-\mathrm{C}(1)-\mathrm{C}(2)$ & $115.5(5)$ & $\mathrm{C}(17)-\mathrm{C}(16)-\mathrm{C}(26)$ & $111.1(5)$ \\
\hline$C(16)-C(25)$ & $1.563(8)$ & $C(6)-C(1)-C(2)$ & $120.1(5)$ & $C(15)-C(16)-C(26)$ & $111.6(5)$ \\
\hline $\mathrm{C}(17)-\mathrm{C}(18)$ & $1.387(8)$ & $\mathrm{O}(2)-\mathrm{C}(2)-\mathrm{C}(1)$ & $119.5(5)$ & $\mathrm{C}(17)-\mathrm{C}(16)-\mathrm{C}(25)$ & $107.8(4)$ \\
\hline$C(17)-C(22)$ & $1.399(7)$ & $\mathrm{O}(2)-\mathrm{C}(2)-\mathrm{C}(3)$ & $120.1(5)$ & $C(15)-C(16)-C(25)$ & $108.5(5)$ \\
\hline$C(18)-C(19)$ & $1.392(8)$ & $C(1)-C(2)-C(3)$ & $120.2(5)$ & $C(26)-C(16)-C(25)$ & $108.4(5)$ \\
\hline $\mathrm{C}(18)-\mathrm{H}(18)$ & 0.9500 & $\mathrm{O}(3)-\mathrm{C}(3)-\mathrm{C}(4)$ & $125.7(5)$ & $\mathrm{C}(18)-\mathrm{C}(17)-\mathrm{C}(22)$ & $117.2(5)$ \\
\hline$C(19)-C(20)$ & $1.416(8)$ & $\mathrm{O}(3)-\mathrm{C}(3)-\mathrm{C}(2)$ & $115.0(5)$ & $\mathrm{C}(18)-\mathrm{C}(17)-\mathrm{C}(16)$ & $124.9(5)$ \\
\hline
\end{tabular}




\begin{tabular}{|c|c|c|c|c|c|}
\hline$C(22)-C(17)-C(16)$ & $117.7(5)$ & $\mathrm{C}(16)-\mathrm{C}(25)-\mathrm{H}(25 \mathrm{~B})$ & 109.5 & $\mathrm{C}(32)-\mathrm{C}(31)-\mathrm{C}(30)$ & $119.2(5)$ \\
\hline $\mathrm{C}(17)-\mathrm{C}(18)-\mathrm{C}(19)$ & $122.6(5)$ & $\mathrm{H}(25 \mathrm{~A})-\mathrm{C}(25)-\mathrm{H}(25 \mathrm{~B})$ & 109.5 & $\mathrm{C}(32)-\mathrm{C}(31)-\mathrm{H}(31)$ & 120.4 \\
\hline $\mathrm{C}(17)-\mathrm{C}(18)-\mathrm{H}(18)$ & 118.7 & $\mathrm{C}(16)-\mathrm{C}(25)-\mathrm{H}(25 \mathrm{C})$ & 109.5 & $\mathrm{C}(30)-\mathrm{C}(31)-\mathrm{H}(31)$ & 120.4 \\
\hline $\mathrm{C}(19)-\mathrm{C}(18)-\mathrm{H}(18)$ & 118.7 & $\mathrm{H}(25 \mathrm{~A})-\mathrm{C}(25)-\mathrm{H}(25 \mathrm{C})$ & 109.5 & $\mathrm{C}(31)-\mathrm{C}(32)-\mathrm{C}(33)$ & $120.8(5)$ \\
\hline $\mathrm{C}(18)-\mathrm{C}(19)-\mathrm{C}(20)$ & $118.8(5)$ & $\mathrm{H}(25 \mathrm{~B})-\mathrm{C}(25)-\mathrm{H}(25 \mathrm{C})$ & 109.5 & $\mathrm{C}(31)-\mathrm{C}(32)-\mathrm{H}(32)$ & 119.6 \\
\hline$C(18)-C(19)-C(27)$ & $121.2(5)$ & $\mathrm{C}(16)-\mathrm{C}(26)-\mathrm{H}(26 \mathrm{~A})$ & 109.5 & $\mathrm{C}(33)-\mathrm{C}(32)-\mathrm{H}(32)$ & 119.6 \\
\hline$C(20)-C(19)-C(27)$ & $119.9(5)$ & $\mathrm{C}(16)-\mathrm{C}(26)-\mathrm{H}(26 \mathrm{~B})$ & 109.5 & $\mathrm{~N}(3)-\mathrm{C}(33)-\mathrm{C}(32)$ & $119.2(5)$ \\
\hline$C(21)-C(20)-C(19)$ & $119.9(5)$ & $\mathrm{H}(26 \mathrm{~A})-\mathrm{C}(26)-\mathrm{H}(26 \mathrm{~B})$ & 109.5 & $\mathrm{~N}(3)-\mathrm{C}(33)-\mathrm{C}(34) \# 1$ & $121.0(5)$ \\
\hline $\mathrm{C}(21)-\mathrm{C}(20)-\mathrm{H}(20)$ & 120.1 & $\mathrm{C}(16)-\mathrm{C}(26)-\mathrm{H}(26 \mathrm{C})$ & 109.5 & $\mathrm{C}(32)-\mathrm{C}(33)-\mathrm{C}(34) \# 1$ & $119.8(5)$ \\
\hline$C(19)-C(20)-H(20)$ & 120.1 & $\mathrm{H}(26 \mathrm{~A})-\mathrm{C}(26)-\mathrm{H}(26 \mathrm{C})$ & 109.5 & $\mathrm{~N}(3)-\mathrm{C}(34)-\mathrm{C}(33) \# 1$ & $120.1(5)$ \\
\hline $\mathrm{C}(20)-\mathrm{C}(21)-\mathrm{C}(22)$ & $119.9(5)$ & $\mathrm{H}(26 \mathrm{~B})-\mathrm{C}(26)-\mathrm{H}(26 \mathrm{C})$ & 109.5 & $\mathrm{~N}(3)-\mathrm{C}(34)-\mathrm{C}(29) \# 1$ & $121.6(5)$ \\
\hline $\mathrm{C}(20)-\mathrm{C}(21)-\mathrm{N}(2)$ & $124.9(5)$ & $\mathrm{C}(19)-\mathrm{C}(27)-\mathrm{H}(27 \mathrm{~A})$ & 109.5 & $\mathrm{C}(33) \# 1-\mathrm{C}(34)-\mathrm{C}(29) \# 1$ & $118.3(5)$ \\
\hline$C(22)-C(21)-N(2)$ & $115.0(5)$ & $\mathrm{C}(19)-\mathrm{C}(27)-\mathrm{H}(27 \mathrm{~B})$ & 109.5 & $\mathrm{Cl}(3)-\mathrm{C}(1 \mathrm{~S})-\mathrm{Cl}(2)$ & $110.2(3)$ \\
\hline $\mathrm{O}(5)-\mathrm{C}(22)-\mathrm{C}(17)$ & $122.0(5)$ & H(27A)-C(27)-H(27B) & 109.5 & $\mathrm{Cl}(3)-\mathrm{C}(1 \mathrm{~S})-\mathrm{Cl}(1)$ & $109.6(3)$ \\
\hline $\mathrm{O}(5)-\mathrm{C}(22)-\mathrm{C}(21)$ & $116.4(4)$ & $\mathrm{C}(19)-\mathrm{C}(27)-\mathrm{H}(27 \mathrm{C})$ & 109.5 & $\mathrm{Cl}(2)-\mathrm{C}(1 \mathrm{~S})-\mathrm{Cl}(1)$ & $109.0(3)$ \\
\hline $\mathrm{C}(17)-\mathrm{C}(22)-\mathrm{C}(21)$ & $121.6(5)$ & $\mathrm{H}(27 \mathrm{~A})-\mathrm{C}(27)-\mathrm{H}(27 \mathrm{C})$ & 109.5 & $\mathrm{Cl}(3)-\mathrm{C}(1 \mathrm{~S})-\mathrm{H}(1 \mathrm{~S})$ & 109.3 \\
\hline $\mathrm{O}(5)-\mathrm{C}(23)-\mathrm{C}(15)$ & $121.5(5)$ & H(27B)-C(27)-H(27C) & 109.5 & $\mathrm{Cl}(2)-\mathrm{C}(1 \mathrm{~S})-\mathrm{H}(1 \mathrm{~S})$ & 109.3 \\
\hline $\mathrm{O}(5)-\mathrm{C}(23)-\mathrm{C}(11)$ & $116.2(4)$ & $\mathrm{O}(6)-\mathrm{C}(28)-\mathrm{N}(2)$ & $124.3(5)$ & $\mathrm{Cl}(1)-\mathrm{C}(1 \mathrm{~S})-\mathrm{H}(1 \mathrm{~S})$ & 109.3 \\
\hline$C(15)-C(23)-C(11)$ & $122.3(5)$ & $\mathrm{O}(6)-\mathrm{C}(28)-\mathrm{C}(29)$ & $120.6(5)$ & $\mathrm{Cl}(4)-\mathrm{C}(2 \mathrm{~S})-\mathrm{Cl}(5)$ & $112.1(4)$ \\
\hline $\mathrm{C}(13)-\mathrm{C}(24)-\mathrm{H}(24 \mathrm{~A})$ & 109.5 & $\mathrm{~N}(2)-\mathrm{C}(28)-\mathrm{C}(29)$ & $115.1(5)$ & $\mathrm{Cl}(4)-\mathrm{C}(2 \mathrm{~S})-\mathrm{Cl}\left(6^{\prime}\right)$ & $114.8(10)$ \\
\hline $\mathrm{C}(13)-\mathrm{C}(24)-\mathrm{H}(24 \mathrm{~B})$ & 109.5 & $\mathrm{C}(30)-\mathrm{C}(29)-\mathrm{C}(34) \# 1$ & $119.0(5)$ & $\mathrm{Cl}(5)-\mathrm{C}(2 \mathrm{~S})-\mathrm{Cl}\left(6^{\prime}\right)$ & $128.3(11)$ \\
\hline $\mathrm{H}(24 \mathrm{~A})-\mathrm{C}(24)-\mathrm{H}(24 \mathrm{~B})$ & 109.5 & $C(30)-C(29)-C(28)$ & $114.8(5)$ & $\mathrm{Cl}(4)-\mathrm{C}(2 \mathrm{~S})-\mathrm{Cl}(6)$ & $110.0(4)$ \\
\hline $\mathrm{C}(13)-\mathrm{C}(24)-\mathrm{H}(24 \mathrm{C})$ & 109.5 & $\mathrm{C}(34) \# 1-\mathrm{C}(29)-\mathrm{C}(28)$ & $126.2(5)$ & $\mathrm{Cl}(5)-\mathrm{C}(2 \mathrm{~S})-\mathrm{Cl}(6)$ & $109.4(4)$ \\
\hline $\mathrm{H}(24 \mathrm{~A})-\mathrm{C}(24)-\mathrm{H}(24 \mathrm{C})$ & 109.5 & $\mathrm{C}(29)-\mathrm{C}(30)-\mathrm{C}(31)$ & $122.9(5)$ & $\mathrm{Cl}(4)-\mathrm{C}(2 \mathrm{~S})-\mathrm{H}(2 \mathrm{~S})$ & 108.4 \\
\hline $\mathrm{H}(24 \mathrm{~B})-\mathrm{C}(24)-\mathrm{H}(24 \mathrm{C})$ & 109.5 & $\mathrm{C}(29)-\mathrm{C}(30)-\mathrm{H}(30)$ & 118.5 & $\mathrm{Cl}(5)-\mathrm{C}(2 \mathrm{~S})-\mathrm{H}(2 \mathrm{~S})$ & 108.4 \\
\hline $\mathrm{C}(16)-\mathrm{C}(25)-\mathrm{H}(25 \mathrm{~A})$ & 109.5 & $\mathrm{C}(31)-\mathrm{C}(30)-\mathrm{H}(30)$ & 118.5 & $\mathrm{Cl}(6)-\mathrm{C}(2 \mathrm{~S})-\mathrm{H}(2 \mathrm{~S})$ & 108.4 \\
\hline
\end{tabular}

Symmetry transformations used to generate equivalent atoms:

$\# 1-\mathrm{x}+1,-\mathrm{y}+1,-\mathrm{z}+1$ 
Table S-4. Anisotropic displacement parameters $\left(\AA^{2} \times 10^{3}\right)$ for vb7519. The anisotropic displacement factor exponent takes the form: $-2{ }^{2}\left[\mathrm{~h}^{2} \mathrm{a}^{* 2} \mathrm{U}^{11}+\ldots+2 \mathrm{~h} \mathrm{k} \mathrm{a}^{*} \mathrm{~b}^{*} \mathrm{U}^{12}\right]$

\begin{tabular}{|c|c|c|c|c|c|c|}
\hline & $\mathrm{U}^{11}$ & $\mathrm{U}^{22}$ & $\mathrm{U}^{33}$ & $\mathrm{U}^{23}$ & $\mathrm{U}^{13}$ & $\mathrm{U}^{12}$ \\
\hline $\mathrm{O}(1)$ & $26(2)$ & $32(2)$ & $49(2)$ & $1(2)$ & $6(2)$ & $16(2)$ \\
\hline $\mathrm{O}(2)$ & $32(2)$ & $23(2)$ & $46(2)$ & $8(2)$ & $15(2)$ & $14(2)$ \\
\hline $\mathrm{O}(3)$ & $36(2)$ & $29(2)$ & $36(2)$ & $1(2)$ & $4(2)$ & $16(2)$ \\
\hline $\mathrm{O}(4)$ & $51(3)$ & $42(2)$ & $36(2)$ & $2(2)$ & $3(2)$ & $33(2)$ \\
\hline $\mathrm{O}(5)$ & $33(2)$ & $22(2)$ & $33(2)$ & $6(2)$ & $6(2)$ & $16(2)$ \\
\hline $\mathrm{O}(6)$ & $30(2)$ & $33(2)$ & $39(2)$ & $2(2)$ & $0(2)$ & $16(2)$ \\
\hline $\mathrm{N}(1)$ & $27(2)$ & $25(2)$ & $32(2)$ & $9(2)$ & $7(2)$ & $14(2)$ \\
\hline $\mathrm{N}(2)$ & $33(2)$ & $22(2)$ & $27(2)$ & $4(2)$ & $7(2)$ & $19(2)$ \\
\hline $\mathrm{N}(3)$ & $30(2)$ & $23(2)$ & $35(2)$ & $10(2)$ & $12(2)$ & $15(2)$ \\
\hline$C(1)$ & $25(3)$ & $27(3)$ & $40(3)$ & $10(2)$ & $13(2)$ & $12(2)$ \\
\hline $\mathrm{C}(2)$ & $31(3)$ & $25(3)$ & $37(3)$ & $10(2)$ & $13(2)$ & $17(2)$ \\
\hline$C(3)$ & $31(3)$ & $25(3)$ & $36(3)$ & $10(2)$ & $14(2)$ & $14(2)$ \\
\hline $\mathrm{C}(4)$ & $30(3)$ & $31(3)$ & $33(3)$ & $13(2)$ & $10(2)$ & $17(2)$ \\
\hline$C(5)$ & $34(3)$ & $26(3)$ & $30(3)$ & $11(2)$ & $11(2)$ & $17(2)$ \\
\hline$C(6)$ & $31(3)$ & $24(3)$ & $32(3)$ & $10(2)$ & $11(2)$ & $13(2)$ \\
\hline$C(7)$ & $28(3)$ & $36(3)$ & $52(4)$ & $3(3)$ & 7(3) & $14(3)$ \\
\hline $\mathrm{C}(8)$ & $44(3)$ & $32(3)$ & $48(3)$ & $16(3)$ & 19(3) & $24(3)$ \\
\hline$C(9)$ & $44(4)$ & $31(3)$ & $39(3)$ & $5(2)$ & 1(3) & $19(3)$ \\
\hline $\mathrm{C}(10)$ & $37(3)$ & $27(3)$ & $35(3)$ & $12(2)$ & $11(2)$ & $19(2)$ \\
\hline $\mathrm{C}(11)$ & $25(3)$ & $15(2)$ & $36(3)$ & $9(2)$ & $7(2)$ & $10(2)$ \\
\hline$C(12)$ & $27(3)$ & $21(2)$ & $38(3)$ & $11(2)$ & $11(2)$ & $11(2)$ \\
\hline $\mathrm{C}(13)$ & $29(3)$ & $21(2)$ & $45(3)$ & $16(2)$ & $15(2)$ & $13(2)$ \\
\hline $\mathrm{C}(14)$ & $27(3)$ & $19(2)$ & $46(3)$ & $8(2)$ & $14(2)$ & $11(2)$ \\
\hline $\mathrm{C}(15)$ & $26(3)$ & $17(2)$ & $41(3)$ & $8(2)$ & $11(2)$ & $12(2)$ \\
\hline$C(16)$ & $31(3)$ & $24(3)$ & $40(3)$ & $8(2)$ & $14(2)$ & $17(2)$ \\
\hline $\mathrm{C}(17)$ & $32(3)$ & $21(3)$ & $42(3)$ & $11(2)$ & $16(2)$ & $14(2)$ \\
\hline $\mathrm{C}(18)$ & $38(3)$ & $23(3)$ & $38(3)$ & $6(2)$ & $16(2)$ & $16(2)$ \\
\hline $\mathrm{C}(19)$ & $33(3)$ & $22(3)$ & $38(3)$ & $6(2)$ & $11(2)$ & $12(2)$ \\
\hline $\mathrm{C}(20)$ & $34(3)$ & $29(3)$ & $38(3)$ & $11(2)$ & $7(2)$ & $18(2)$ \\
\hline $\mathrm{C}(21)$ & $34(3)$ & $23(3)$ & $33(3)$ & $9(2)$ & $13(2)$ & $16(2)$ \\
\hline $\mathrm{C}(22)$ & $31(3)$ & $23(3)$ & $32(3)$ & $10(2)$ & $13(2)$ & $14(2)$ \\
\hline $\mathrm{C}(23)$ & $27(3)$ & $16(2)$ & $39(3)$ & $5(2)$ & $9(2)$ & $11(2)$ \\
\hline$C(24)$ & $37(3)$ & $33(3)$ & $43(3)$ & $17(2)$ & $17(3)$ & $23(3)$ \\
\hline$C(25)$ & $33(3)$ & $36(3)$ & $39(3)$ & $13(2)$ & $9(2)$ & $16(3)$ \\
\hline $\mathrm{C}(26)$ & $50(4)$ & $28(3)$ & $40(3)$ & $4(2)$ & 11(3) & $25(3)$ \\
\hline $\mathrm{C}(27)$ & $47(4)$ & $31(3)$ & $39(3)$ & $4(2)$ & $7(3)$ & $20(3)$ \\
\hline $\mathrm{C}(28)$ & $31(3)$ & $22(3)$ & $34(3)$ & $9(2)$ & $13(2)$ & $13(2)$ \\
\hline $\mathrm{C}(29)$ & $27(3)$ & $24(3)$ & $34(3)$ & $10(2)$ & $12(2)$ & $13(2)$ \\
\hline $\mathrm{C}(30)$ & $27(3)$ & $27(3)$ & $39(3)$ & $9(2)$ & $6(2)$ & $13(2)$ \\
\hline $\mathrm{C}(31)$ & $29(3)$ & $28(3)$ & $38(3)$ & $8(2)$ & $11(2)$ & $14(2)$ \\
\hline$C(32)$ & $31(3)$ & $25(3)$ & $32(3)$ & $9(2)$ & $14(2)$ & $17(2)$ \\
\hline $\mathrm{C}(33)$ & $34(3)$ & $20(2)$ & $31(3)$ & $11(2)$ & $15(2)$ & $15(2)$ \\
\hline C(34) & $28(3)$ & $22(2)$ & $36(3)$ & $12(2)$ & $16(2)$ & $16(2)$ \\
\hline $\mathrm{C}(1 \mathrm{~S})$ & $38(3)$ & $30(3)$ & $39(3)$ & $12(2)$ & 13(3) & 11(3) \\
\hline $\mathrm{C}(2 \mathrm{~S})$ & $44(4)$ & 41(3) & $47(4)$ & $15(3)$ & $9(3)$ & $20(3)$ \\
\hline $\mathrm{Cl}(1)$ & $42(1)$ & $43(1)$ & $50(1)$ & $10(1)$ & $13(1)$ & $22(1)$ \\
\hline $\mathrm{Cl}(2)$ & $46(1)$ & $30(1)$ & $54(1)$ & $10(1)$ & $22(1)$ & 11(1) \\
\hline $\mathrm{Cl}(3)$ & $51(1)$ & $44(1)$ & $45(1)$ & $18(1)$ & $7(1)$ & $10(1)$ \\
\hline $\mathrm{Cl}(4)$ & $51(1)$ & $42(1)$ & $62(1)$ & $22(1)$ & 21(1) & $16(1)$ \\
\hline $\mathrm{Cl}(5)$ & $47(1)$ & $38(1)$ & $63(1)$ & $7(1)$ & $10(1)$ & $16(1)$ \\
\hline $\mathrm{Cl}(6)$ & $48(1)$ & $65(1)$ & $75(2)$ & $38(1)$ & $32(1)$ & $33(1)$ \\
\hline $\mathrm{Cl}\left(6^{\prime}\right)$ & $48(1)$ & $65(1)$ & $75(2)$ & $38(1)$ & $32(1)$ & $33(1)$ \\
\hline
\end{tabular}

S-36 
Table S-5. Hydrogen coordinates ( x 104) and isotropic displacement parameters $\left(\AA^{2} \times 10^{3}\right)$ for vb7519.

\begin{tabular}{|c|c|c|c|c|}
\hline & $\mathrm{x}$ & $y$ & $\mathrm{z}$ & $\mathrm{U}(\mathrm{eq})$ \\
\hline $\mathrm{H}(1)$ & 4777 & 2155 & 5187 & 33 \\
\hline $\mathrm{H}(2)$ & 3897 & 2626 & 3551 & 32 \\
\hline $\mathrm{H}(4)$ & 6862 & 4447 & 7804 & 35 \\
\hline $\mathrm{H}(6)$ & 3015 & 2554 & 5608 & 33 \\
\hline $\mathrm{H}(7 \mathrm{~A})$ & 914 & 2714 & 4660 & 62 \\
\hline $\mathrm{H}(7 \mathrm{~B})$ & -696 & 2683 & 4866 & 62 \\
\hline $\mathrm{H}(7 \mathrm{C})$ & 351 & 2127 & 5370 & 62 \\
\hline $\mathrm{H}(8 \mathrm{~A})$ & 2635 & 6099 & 6458 & 56 \\
\hline $\mathrm{H}(8 \mathrm{~B})$ & 4148 & 6623 & 7370 & 56 \\
\hline $\mathrm{H}(8 \mathrm{C})$ & 2493 & 6692 & 7422 & 56 \\
\hline $\mathrm{H}(9 \mathrm{~A})$ & 7914 & 6174 & 8672 & 60 \\
\hline $\mathrm{H}(9 \mathrm{~B})$ & 7173 & 5533 & 9302 & 60 \\
\hline $\mathrm{H}(9 \mathrm{C})$ & 7429 & 6737 & 9510 & 60 \\
\hline $\mathrm{H}(12)$ & 7171 & 1131 & 6452 & 33 \\
\hline $\mathrm{H}(14)$ & 7846 & -495 & 4188 & 36 \\
\hline $\mathrm{H}(18)$ & 4410 & -595 & 1063 & 38 \\
\hline $\mathrm{H}(20)$ & 1699 & 1163 & 1209 & 39 \\
\hline $\mathrm{H}(24 \mathrm{~A})$ & 8521 & 34 & 6657 & 50 \\
\hline H(24B) & 7927 & -993 & 5793 & 50 \\
\hline $\mathrm{H}(24 \mathrm{C})$ & 9566 & -84 & 5981 & 50 \\
\hline $\mathrm{H}(25 \mathrm{~A})$ & 7989 & 1761 & 3017 & 53 \\
\hline $\mathrm{H}(25 \mathrm{~B})$ & 7843 & 951 & 2074 & 53 \\
\hline $\mathrm{H}(25 \mathrm{C})$ & 8913 & 978 & 3055 & 53 \\
\hline $\mathrm{H}(26 \mathrm{~A})$ & 7386 & -942 & 2740 & 58 \\
\hline $\mathrm{H}(26 \mathrm{~B})$ & 6437 & -926 & 1744 & 58 \\
\hline $\mathrm{H}(26 \mathrm{C})$ & 5503 & -1367 & 2408 & 58 \\
\hline $\mathrm{H}(27 \mathrm{~A})$ & 2711 & -509 & -378 & 60 \\
\hline $\mathrm{H}(27 \mathrm{~B})$ & 1564 & 124 & -299 & 60 \\
\hline $\mathrm{H}(27 \mathrm{C})$ & 1145 & -982 & -118 & 60 \\
\hline $\mathrm{H}(30)$ & 250 & 3722 & 2752 & 37 \\
\hline $\mathrm{H}(31)$ & 345 & 5303 & 3686 & 37 \\
\hline $\mathrm{H}(32)$ & 2535 & 6301 & 4964 & 33 \\
\hline $\mathrm{H}(1 \mathrm{~S})$ & 6582 & 3872 & 898 & 43 \\
\hline $\mathrm{H}(2 \mathrm{~S})$ & 10331 & 7114 & 2428 & 52 \\
\hline
\end{tabular}


Table S-6. Torsion angles $\left[{ }^{\circ}\right]$ for vb7519.

\begin{tabular}{|c|c|c|c|}
\hline $\mathrm{C}(7)-\mathrm{O}(1)-\mathrm{C}(1)-\mathrm{C}(6)$ & $2.3(8)$ & $\mathrm{C}(26)-\mathrm{C}(16)-\mathrm{C}(17)-\mathrm{C}(22)$ & $156.6(5)$ \\
\hline $\mathrm{C}(7)-\mathrm{O}(1)-\mathrm{C}(1)-\mathrm{C}(2)$ & $-176.4(5)$ & $\mathrm{C}(25)-\mathrm{C}(16)-\mathrm{C}(17)-\mathrm{C}(22)$ & $-84.8(6)$ \\
\hline $\mathrm{C}(8)-\mathrm{O}(2)-\mathrm{C}(2)-\mathrm{C}(1)$ & $-105.8(6)$ & $\mathrm{C}(22)-\mathrm{C}(17)-\mathrm{C}(18)-\mathrm{C}(19)$ & $0.9(8)$ \\
\hline $\mathrm{C}(8)-\mathrm{O}(2)-\mathrm{C}(2)-\mathrm{C}(3)$ & $80.0(6)$ & $\mathrm{C}(16)-\mathrm{C}(17)-\mathrm{C}(18)-\mathrm{C}(19)$ & $-173.3(5)$ \\
\hline $\mathrm{O}(1)-\mathrm{C}(1)-\mathrm{C}(2)-\mathrm{O}(2)$ & $4.2(8)$ & $\mathrm{C}(17)-\mathrm{C}(18)-\mathrm{C}(19)-\mathrm{C}(20)$ & $1.1(9)$ \\
\hline $\mathrm{C}(6)-\mathrm{C}(1)-\mathrm{C}(2)-\mathrm{O}(2)$ & $-174.5(5)$ & $\mathrm{C}(17)-\mathrm{C}(18)-\mathrm{C}(19)-\mathrm{C}(27)$ & $178.6(6)$ \\
\hline $\mathrm{O}(1)-\mathrm{C}(1)-\mathrm{C}(2)-\mathrm{C}(3)$ & $178.3(5)$ & $\mathrm{C}(18)-\mathrm{C}(19)-\mathrm{C}(20)-\mathrm{C}(21)$ & $-1.2(9)$ \\
\hline$C(6)-C(1)-C(2)-C(3)$ & $-0.4(8)$ & $\mathrm{C}(27)-\mathrm{C}(19)-\mathrm{C}(20)-\mathrm{C}(21)$ & $-178.7(6)$ \\
\hline $\mathrm{C}(9)-\mathrm{O}(3)-\mathrm{C}(3)-\mathrm{C}(4)$ & $-3.2(8)$ & $\mathrm{C}(19)-\mathrm{C}(20)-\mathrm{C}(21)-\mathrm{C}(22)$ & $-0.7(9)$ \\
\hline $\mathrm{C}(9)-\mathrm{O}(3)-\mathrm{C}(3)-\mathrm{C}(2)$ & $176.8(5)$ & $\mathrm{C}(19)-\mathrm{C}(20)-\mathrm{C}(21)-\mathrm{N}(2)$ & $174.1(5)$ \\
\hline $\mathrm{O}(2)-\mathrm{C}(2)-\mathrm{C}(3)-\mathrm{O}(3)$ & $-5.2(8)$ & $\mathrm{C}(28)-\mathrm{N}(2)-\mathrm{C}(21)-\mathrm{C}(20)$ & $8.6(9)$ \\
\hline $\mathrm{C}(1)-\mathrm{C}(2)-\mathrm{C}(3)-\mathrm{O}(3)$ & $-179.3(5)$ & $\mathrm{C}(28)-\mathrm{N}(2)-\mathrm{C}(21)-\mathrm{C}(22)$ & $-176.3(5)$ \\
\hline $\mathrm{O}(2)-\mathrm{C}(2)-\mathrm{C}(3)-\mathrm{C}(4)$ & $174.7(5)$ & $\mathrm{C}(23)-\mathrm{O}(5)-\mathrm{C}(22)-\mathrm{C}(17)$ & $-25.8(7)$ \\
\hline$C(1)-C(2)-C(3)-C(4)$ & $0.7(8)$ & $\mathrm{C}(23)-\mathrm{O}(5)-\mathrm{C}(22)-\mathrm{C}(21)$ & $156.3(5)$ \\
\hline $\mathrm{O}(3)-\mathrm{C}(3)-\mathrm{C}(4)-\mathrm{C}(5)$ & $179.2(5)$ & $\mathrm{C}(18)-\mathrm{C}(17)-\mathrm{C}(22)-\mathrm{O}(5)$ & $179.3(5)$ \\
\hline$C(2)-C(3)-C(4)-C(5)$ & $-0.7(8)$ & $\mathrm{C}(16)-\mathrm{C}(17)-\mathrm{C}(22)-\mathrm{O}(5)$ & $-6.1(8)$ \\
\hline$C(3)-C(4)-C(5)-C(6)$ & $0.6(8)$ & $\mathrm{C}(18)-\mathrm{C}(17)-\mathrm{C}(22)-\mathrm{C}(21)$ & $-2.9(8)$ \\
\hline$C(3)-C(4)-C(5)-C(10)$ & $-179.5(5)$ & $\mathrm{C}(16)-\mathrm{C}(17)-\mathrm{C}(22)-\mathrm{C}(21)$ & $171.8(5)$ \\
\hline $\mathrm{O}(1)-\mathrm{C}(1)-\mathrm{C}(6)-\mathrm{C}(5)$ & $-178.3(5)$ & $\mathrm{C}(20)-\mathrm{C}(21)-\mathrm{C}(22)-\mathrm{O}(5)$ & $-179.2(5)$ \\
\hline$C(2)-C(1)-C(6)-C(5)$ & $0.3(8)$ & $\mathrm{N}(2)-\mathrm{C}(21)-\mathrm{C}(22)-\mathrm{O}(5)$ & $5.5(7)$ \\
\hline$C(4)-C(5)-C(6)-C(1)$ & $-0.4(8)$ & $\mathrm{C}(20)-\mathrm{C}(21)-\mathrm{C}(22)-\mathrm{C}(17)$ & $2.8(8)$ \\
\hline$C(10)-C(5)-C(6)-C(1)$ & $179.7(5)$ & $\mathrm{N}(2)-\mathrm{C}(21)-\mathrm{C}(22)-\mathrm{C}(17)$ & $-172.5(5)$ \\
\hline $\mathrm{C}(11)-\mathrm{N}(1)-\mathrm{C}(10)-\mathrm{O}(4)$ & $1.0(9)$ & $\mathrm{C}(22)-\mathrm{O}(5)-\mathrm{C}(23)-\mathrm{C}(15)$ & $27.7(7)$ \\
\hline $\mathrm{C}(11)-\mathrm{N}(1)-\mathrm{C}(10)-\mathrm{C}(5)$ & $-179.4(5)$ & $\mathrm{C}(22)-\mathrm{O}(5)-\mathrm{C}(23)-\mathrm{C}(11)$ & $-151.6(5)$ \\
\hline$C(4)-C(5)-C(10)-O(4)$ & $24.0(8)$ & $\mathrm{C}(14)-\mathrm{C}(15)-\mathrm{C}(23)-\mathrm{O}(5)$ & $178.0(5)$ \\
\hline $\mathrm{C}(6)-\mathrm{C}(5)-\mathrm{C}(10)-\mathrm{O}(4)$ & $-156.1(6)$ & $\mathrm{C}(16)-\mathrm{C}(15)-\mathrm{C}(23)-\mathrm{O}(5)$ & $2.4(8)$ \\
\hline $\mathrm{C}(4)-\mathrm{C}(5)-\mathrm{C}(10)-\mathrm{N}(1)$ & $-155.6(5)$ & $\mathrm{C}(14)-\mathrm{C}(15)-\mathrm{C}(23)-\mathrm{C}(11)$ & $-2.8(8)$ \\
\hline$C(6)-C(5)-C(10)-N(1)$ & $24.4(8)$ & $\mathrm{C}(16)-\mathrm{C}(15)-\mathrm{C}(23)-\mathrm{C}(11)$ & $-178.4(5)$ \\
\hline $\mathrm{C}(10)-\mathrm{N}(1)-\mathrm{C}(11)-\mathrm{C}(12)$ & $26.0(8)$ & $\mathrm{C}(12)-\mathrm{C}(11)-\mathrm{C}(23)-\mathrm{O}(5)$ & $-177.7(5)$ \\
\hline $\mathrm{C}(10)-\mathrm{N}(1)-\mathrm{C}(11)-\mathrm{C}(23)$ & $-155.2(5)$ & $\mathrm{N}(1)-\mathrm{C}(11)-\mathrm{C}(23)-\mathrm{O}(5)$ & $3.5(7)$ \\
\hline$C(23)-C(11)-C(12)-C(13)$ & $-1.1(8)$ & $\mathrm{C}(12)-\mathrm{C}(11)-\mathrm{C}(23)-\mathrm{C}(15)$ & $3.0(8)$ \\
\hline $\mathrm{N}(1)-\mathrm{C}(11)-\mathrm{C}(12)-\mathrm{C}(13)$ & $177.7(5)$ & $\mathrm{N}(1)-\mathrm{C}(11)-\mathrm{C}(23)-\mathrm{C}(15)$ & $-175.8(5)$ \\
\hline$C(11)-C(12)-C(13)-C(14)$ & $-1.0(8)$ & $\mathrm{C}(21)-\mathrm{N}(2)-\mathrm{C}(28)-\mathrm{O}(6)$ & $8.4(9)$ \\
\hline $\mathrm{C}(11)-\mathrm{C}(12)-\mathrm{C}(13)-\mathrm{C}(24)$ & $-179.6(5)$ & $\mathrm{C}(21)-\mathrm{N}(2)-\mathrm{C}(28)-\mathrm{C}(29)$ & $-170.8(5)$ \\
\hline$C(12)-C(13)-C(14)-C(15)$ & $1.2(8)$ & $\mathrm{O}(6)-\mathrm{C}(28)-\mathrm{C}(29)-\mathrm{C}(30)$ & $5.7(8)$ \\
\hline$C(24)-C(13)-C(14)-C(15)$ & $179.8(5)$ & $\mathrm{N}(2)-\mathrm{C}(28)-\mathrm{C}(29)-\mathrm{C}(30)$ & $-175.1(5)$ \\
\hline$C(13)-C(14)-C(15)-C(23)$ & $0.7(8)$ & $\mathrm{O}(6)-\mathrm{C}(28)-\mathrm{C}(29)-\mathrm{C}(34) \# 1$ & $-172.2(5)$ \\
\hline$C(13)-C(14)-C(15)-C(16)$ & $176.0(5)$ & $\mathrm{N}(2)-\mathrm{C}(28)-\mathrm{C}(29)-\mathrm{C}(34) \# 1$ & $7.0(8)$ \\
\hline$C(23)-C(15)-C(16)-C(17)$ & $-31.3(7)$ & $\mathrm{C}(34) \# 1-\mathrm{C}(29)-\mathrm{C}(30)-\mathrm{C}(31)$ & $-0.7(8)$ \\
\hline$C(14)-C(15)-C(16)-C(17)$ & $153.4(5)$ & $\mathrm{C}(28)-\mathrm{C}(29)-\mathrm{C}(30)-\mathrm{C}(31)$ & $-178.7(5)$ \\
\hline$C(23)-C(15)-C(16)-C(26)$ & $-154.7(5)$ & $\mathrm{C}(29)-\mathrm{C}(30)-\mathrm{C}(31)-\mathrm{C}(32)$ & $0.7(9)$ \\
\hline$C(14)-C(15)-C(16)-C(26)$ & $30.0(7)$ & $\mathrm{C}(30)-\mathrm{C}(31)-\mathrm{C}(32)-\mathrm{C}(33)$ & $0.7(8)$ \\
\hline$C(23)-C(15)-C(16)-C(25)$ & $86.0(6)$ & $\mathrm{C}(34)-\mathrm{N}(3)-\mathrm{C}(33)-\mathrm{C}(32)$ & $178.5(5)$ \\
\hline$C(14)-C(15)-C(16)-C(25)$ & $-89.3(6)$ & $\mathrm{C}(34)-\mathrm{N}(3)-\mathrm{C}(33)-\mathrm{C}(34) \# 1$ & $-1.4(8)$ \\
\hline$C(15)-C(16)-C(17)-C(18)$ & $-152.9(5)$ & $\mathrm{C}(31)-\mathrm{C}(32)-\mathrm{C}(33)-\mathrm{N}(3)$ & $177.9(5)$ \\
\hline$C(26)-C(16)-C(17)-C(18)$ & $-29.2(8)$ & $\mathrm{C}(31)-\mathrm{C}(32)-\mathrm{C}(33)-\mathrm{C}(34) \# 1$ & $-2.2(8)$ \\
\hline$C(25)-C(16)-C(17)-C(18)$ & $89.4(6)$ & $\mathrm{C}(33)-\mathrm{N}(3)-\mathrm{C}(34)-\mathrm{C}(33) \# 1$ & $1.4(8)$ \\
\hline$C(15)-C(16)-C(17)-C(22)$ & $33.0(6)$ & $\mathrm{C}(33)-\mathrm{N}(3)-\mathrm{C}(34)-\mathrm{C}(29) \# 1$ & $-177.9(5)$ \\
\hline
\end{tabular}

Symmetry transformations used to generate equivalent atoms:

$\# 1-\mathrm{x}+1,-\mathrm{y}+1,-\mathrm{z}+1$ 
Table S-7. Hydrogen bonds for vb7519 [ $\AA \AA$ and $\left.{ }^{\circ}\right]$.

\begin{tabular}{|c|c|c|c|c|}
\hline$\overline{\mathrm{D}-\mathrm{H} . . . \mathrm{A}}$ & $\mathrm{d}(\mathrm{D}-\mathrm{H})$ & d(H...A) & $\mathrm{d}(\mathrm{D} \ldots \mathrm{A})$ & $<(\mathrm{DHA})$ \\
\hline $\mathrm{N}(2)-\mathrm{H}(2) \ldots \mathrm{N}(3) \# 1$ & 0.88 & 2.00 & $2.716(6)$ & 137.8 \\
\hline $\mathrm{C}(8)-\mathrm{H}(8 \mathrm{~B}) \ldots \mathrm{O}(3)$ & 0.98 & 2.52 & $3.051(7)$ & 113.9 \\
\hline $\mathrm{C}(9)-\mathrm{H}(9 \mathrm{~B}) \ldots \mathrm{Cl}(3) \# 2$ & 0.98 & 2.92 & $3.833(7)$ & 154.5 \\
\hline $\mathrm{C}(12)-\mathrm{H}(12) \ldots \mathrm{O}(4)$ & 0.95 & 2.35 & $2.878(7)$ & 114.8 \\
\hline $\mathrm{C}(20)-\mathrm{H}(20) \ldots \mathrm{O}(6)$ & 0.95 & 2.29 & $2.890(7)$ & 120.4 \\
\hline $\mathrm{C}(1 \mathrm{~S})-\mathrm{H}(1 \mathrm{~S}) \ldots \mathrm{O}(2) \# 1$ & 1.00 & 2.50 & $3.315(7)$ & 138.3 \\
\hline $\mathrm{C}(1 \mathrm{~S})-\mathrm{H}(1 \mathrm{~S}) \ldots \mathrm{O}(3) \# 1$ & 1.00 & 2.63 & $3.364(7)$ & 130.3 \\
\hline $\mathrm{C}(2 \mathrm{~S})-\mathrm{H}\left(2 \mathrm{~S}^{\wedge} \mathrm{a}\right) \ldots \mathrm{O}(4) \# 3$ & 1.00 & 2.30 & $2.946(8)$ & 121.4 \\
\hline
\end{tabular}

Symmetry transformations used to generate equivalent atoms:

$\# 1-\mathrm{x}+1,-\mathrm{y}+1,-\mathrm{z}+1 \quad \# 2 \mathrm{x}, \mathrm{y}, \mathrm{z}+1 \quad \# 3-\mathrm{x}+2,-\mathrm{y}+1,-\mathrm{z}+1$

Projection view with $30 \%$ probability ellipsoids- solvent molecules omitted for clarity:

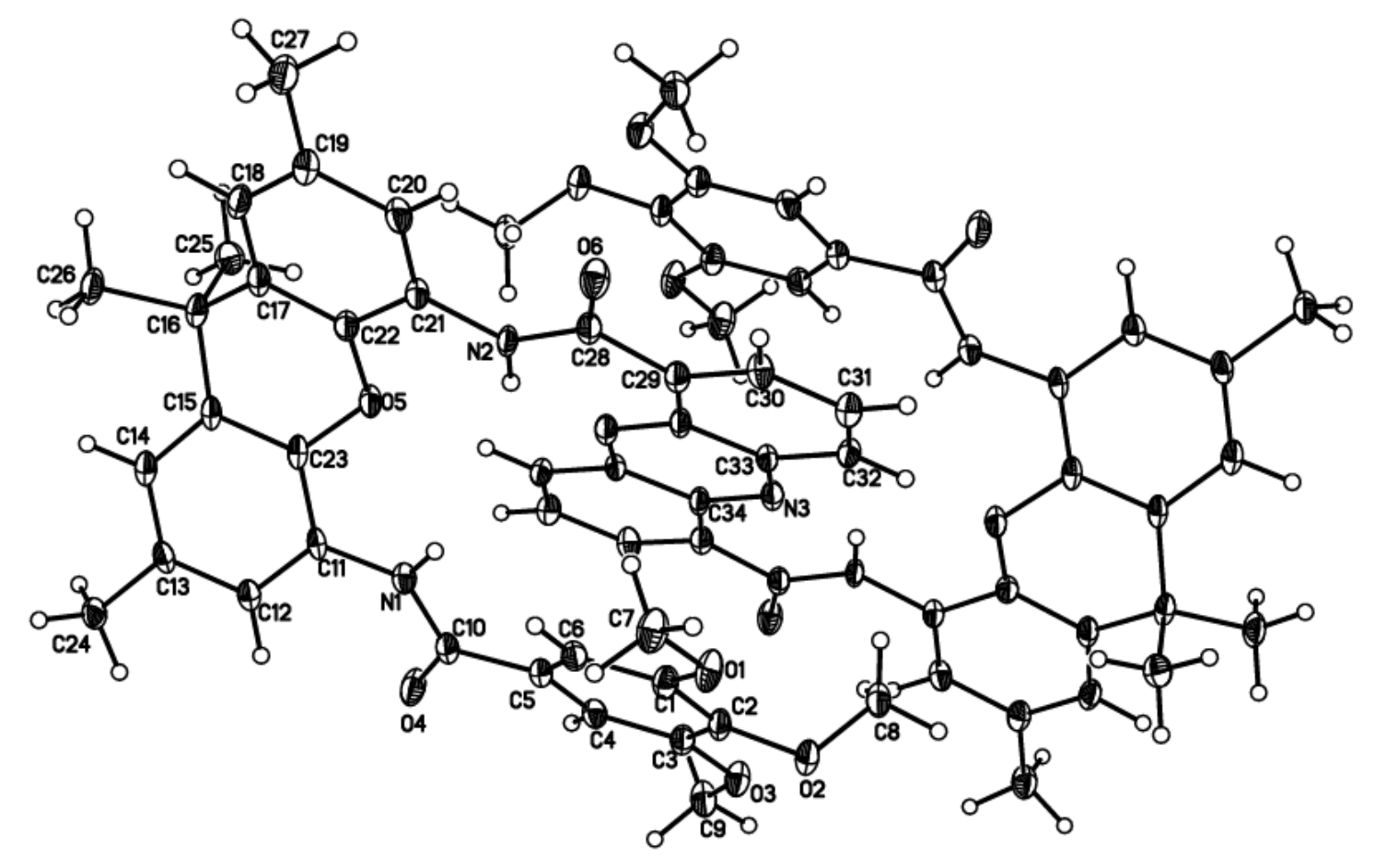




\section{REFERENCES.}

${ }^{1}$ Ferrins, L.; Rahmani, R.; Sykes, M. L.; Jones, A. J.; Avery, V. M.; Teston, E.; Almohaywi, B.; Yin, J. X.; Smith, J.; Hyland, C.; White, K. L.; Ryan, E.; Campbell, M.; Charman, S. A.; Kaiser, M.; Baell, J. B., 3(Oxazolo[4,5-b]pyridin-2-yl)anilides as a novel class of potent inhibitors for the kinetoplastid Trypanosoma brucei, the causative agent for human African trypanosomiasis. Eur. J. Med. Chem. 2013, 66, 450-465.

${ }^{2}$ (a) Rewcastle, G. W.; Denny, W. A., Unequivocal Synthesis of Phenazine-1-Carboxylic Acids: Selective Displacement of Fluorine During Alkaline Borohydride Reduction of N-(2-Fluorophenyl)-3Nitroanthranilic Acids. Synth. Commun. 1987, 17, 1171-1179. (b) For an alternate synthetic route, see: Clark, T. W.; Sperry, J., Biomimetic synthesis of phenazine-1,6-dicarboxylic acid (PDC). Synlett 2012, 23, 2827-2829.

${ }^{3}$ Previously generated and used without isolation: Holmes, R. J.; Abrahams, B. F.; Murray, V.; Denny, W. A.; McFadyen, W. D., A 2D hydrogen-bonded network constructed from large organic dications. J. Mol. Struct. 2010, 975, 186-189.

${ }^{4}$ Herwig, J.; Bohnen, H.; Skutta, P.; Sturm, S.; Van Leeuwen, P. W. N. M.; Bronger, R. Novel diphosphines and a method for their production. WO2002068434A1, 2002.

${ }^{5}$ For a general procedure, see: Komati, R.; Jursic, B. S., Ligand free open air copper (II) mediated aryl formamidation and amination of aryl halides. Tetrahedron Lett. 2014, 55, 1523-1527.

${ }^{6}$ (a) Becke, A. D., Density-functional thermochemistry. III. The role of exact exchange. J. Chem. Phys. 1993, 98, 5648-52. (b) Lee, C.; Yang, W.; Parr, R. G., Development of the Colle-Salvetti correlation-energy formula into a functional of the electron density. Phys. Rev. B: Condens. Matter 1988, 37, 785-9.

${ }^{7}$ Gasparro, F. P.; Kolodny, N. H., NMR determination of the rotational barrier in N, N-dimethylacetamide. A physical chemistry experiment. J. Chem. Educ. 1977, 54, 258-261. 